\title{
Rate Constants for H-atom Transfer Reactions by the BEBO Method
}

\author{
R. L. Brown* \\ National Bureau of Standards, Washington, DC 20234
}

March 17, 1981

\begin{abstract}
A detailed discussion of the calculation of rate constants for hydrogen atom transfer reactions based on the BEBO method is presented. Linear transition state models are used. A computer program using this method for determining rate constants is provided.
\end{abstract}

Key words: absolute chemical rate; $\mathrm{BEBO}$ activation energy; bond-order-bond-energy; chemical rate constants; computer program; hydrogen atom transfer.

\section{CONTENTS}

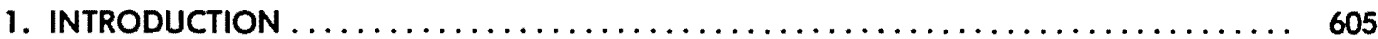

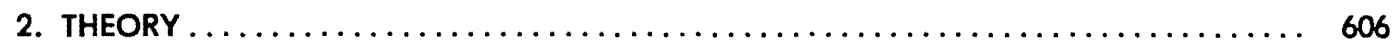

2.1. Absolute Rate Theory \& Transition State Model for BEBO Calculations ......... 606

2.2. BEBO Method $\ldots \ldots \ldots \ldots \ldots \ldots \ldots \ldots \ldots \ldots \ldots \ldots \ldots \ldots \ldots \ldots \ldots \ldots, 610$

2.3. Vibrational Analysis $\ldots \ldots \ldots \ldots \ldots \ldots \ldots \ldots \ldots \ldots \ldots \ldots \ldots \ldots \ldots \ldots \ldots \ldots \ldots, 616$

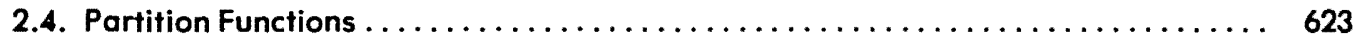

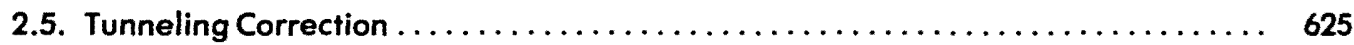

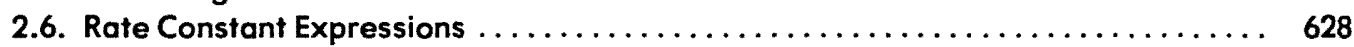

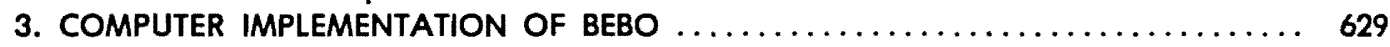

3.1. Description of the Main Program ............................... 629

3.2. Discussion of Subroutine Tunl $\ldots \ldots \ldots \ldots \ldots \ldots \ldots \ldots \ldots \ldots \ldots \ldots \ldots, 630$

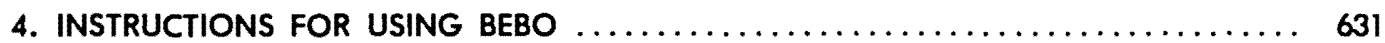

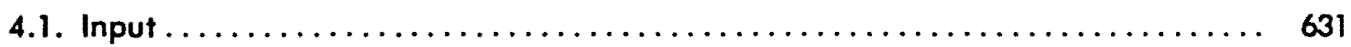

4.2. Output $\ldots \ldots \ldots \ldots \ldots \ldots \ldots \ldots \ldots \ldots \ldots \ldots \ldots \ldots \ldots \ldots \ldots \ldots \ldots \ldots \ldots \ldots \ldots, 634$

5. REFERENCES $\ldots \ldots \ldots \ldots \ldots \ldots \ldots \ldots \ldots \ldots \ldots \ldots \ldots \ldots \ldots \ldots \ldots \ldots \ldots \ldots \ldots \ldots \ldots \ldots \ldots, 634$

6. APPENDIX: Various Triplet Functions $\ldots \ldots \ldots \ldots \ldots \ldots \ldots \ldots \ldots \ldots \ldots \ldots \ldots \ldots, 635$

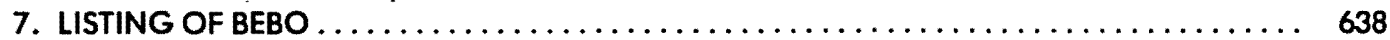

\section{Introduction}

The bond-energy-bond-order (BEBO) method is a procedure for calculating the activation energies of hydrogen transfer reactions from bond energies. When combined with absolute rate theory, it also yields values for the rate constants. It was formulated over 10 years ago by Johnston and Parr [1], ${ }^{1}$ and has since been applied with considerable success to the calculation of a large number of activation energies. Less frequently, it has been used to evaluate rate constants. Although the details of the BEBO method itself have

\footnotetext{
3 Figures in brackets indicate literature references at the end of this paper.

- Center for Thermodynamics and Molecular Science, National Meosurement Laboratory.
} 
been published by Johnston [2], this aspect represents only a relatively small part of a rate constant calculation. The purpose of this report is to give a detailed account, not only of the BEBO method and its theoretical background, but also of the absolute rate theory portion of the calculation. In addition, instructions are provided for the use of a computer program which calculates rate constants based on the BEBO method. The discussion is limited to linear transition state models.

\section{Theory}

\subsection{Absolute Rate Theory \& Transition State Model for BEBO Calculations}

For a bimolecular reaction, $A+B \rightarrow[A B]^{*} \rightarrow$ products, absolute rate theory utilizes the concept of a molecular complex made up of the two reactants. This complex is assumed to be in equilibrium with these reactants. The resulting expression for the classical rate constant $\mathbf{k}_{c l}$ is

$$
\mathbf{k}_{c l}=\frac{k T}{h} \frac{Q^{*}{ }_{c l} e^{-v / k T}}{Q_{c l}^{A} Q_{c l}^{s}}
$$

where $k$ is the Boltzmann constant, $T$ is the absolute temperature, $h$ is Planck's constant, $Q_{c l}^{A}$ and $Q_{c l}^{B}$ are the classical partition functions per unit volume for reactants $A$ and $B, Q_{c l}^{*}$ is the classical partition function per unit volume for the complex, and $V^{*}$ is the potential energy of the complex relative to that of the reactants. The complex contains one unstable vibrational mode whose evolution brings about its dissociation into product fragments. The partition function $Q_{c i}^{*}$ is evaluated with this mode missing. A detailed derivation of eq (1) which explains all its inherent assumptions has been given by Mahan [3]. Quantum mechanical corrections to the partition functions at room temperature and above need be applied only to vibrational factors. For a particular vibration of frequency $\nu_{i}$, the quantum correction $\Gamma_{i}$ is given by the expression

$$
\Gamma_{i}=\frac{u_{i} / 2}{\sinh \left(u_{i} / 2\right)}, \text { where } u_{i}=h v_{i} / k T
$$

We assume that all vibrational modes are independent so that the total quantum correction for a particular species is simply the product of terms given by eq (2), one for each vibrational mode. There is also a quantum correction to the unstable vibrational mode of the complex which we denote by $\Gamma^{*}$. This results from the effect of quantum mechanical tunneling through the potential barrier between reactants and products. It will be considered in detail in section 2.5. Applying these quantum corrections to eq (1) yields the rate expression

$$
\mathbf{k}=\frac{k T}{h} \frac{Q_{c l}^{*}\left\{i\left[\Gamma_{i}^{*}\right\} \Gamma^{*} e^{-v^{*} / k T}\right.}{Q_{c l}^{A}\left\{{ }_{i}^{n} \Gamma_{i}^{A}\right\} Q_{c l}^{B}\left\{{ }_{i}^{n} \Gamma_{i}^{B}\right\}}
$$

The general class of reactions we are considering has the form

$$
A-H+B \cdot-A-H-B-A \cdot+H-B
$$

Radical $B \cdot$ abstracts a hydrogen atom attached to $A$, the net result being the transfer of $H$ from $A$ to $B$. For this system, we take the most general transition state to be linear, having up to 5 mass points. Its structure and the notation which we shall use are shown in figure la. There can be up to four internuclear distances, $R_{s}, R_{b}, R_{c}$, and $R_{d}$. The bonds associated with $R_{a}$ and $R_{d}$ will be assumed to be rigid. (The two vibrational modes involving these bonds will have infinite frequencies and need not be formally included in the calculations.) Thus, there are only two vibrational stretching modes to be considered for this molecule, one of which will be unstable. These modes arise from the stretching of the two central bonds $b$ and $c$ which are shown by dotted lines to indicate their unstable character. Of the five possible masses, $M_{3}$ will normally be that of the hydrogen atom; the other masses will be assigned values in the manner described below. The three angles $\Psi_{2}, \Psi_{3}$, and $\Psi_{4}$ are defined by the bonds $(a, b),(b, c)$, and $(c, d)$ in the plane of the figure while the primed symbols denote the corresponding angles in the plane perpendicular to the figure. Changes in these angles from 


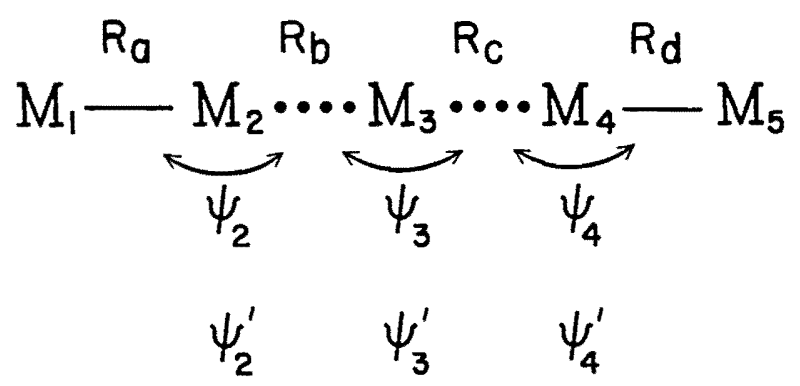

Ficure la. Notation for five mass point linear transition state.

$180^{\circ}$ give rise to three doubly degenerate bending vibrations. To calculate the frequencies needed in eq (3), we require values for the two stretching force constants associated with bonds $b$ and $c$, and three bending force constants arising from the three bond angles. As we shall see, these values can be generated by the BEBO process.

Within the framework of the transition state structure shown in figure la, it is possible to include all types of reactions implied by eq (4) by considering four cases; one having a 3 point transition state, two having 4 point states, and one having a 5 point state. These four cases are shown in figure $1 \mathrm{~b}$. In this figure, the subscript $s$ appearing on the internuclear distances and force constants denote equilibrium values found in reactants or products. Because bonds $a$ and $d$ are assumed to be rigid, their bond distances will always be denoted by the single symbols $R_{a s}$ and $R_{d s}$, respectively. The bond distance between $M_{2}$ and $M_{3}$ goes from $R_{b s}$ to $R_{b}$ in the transition state, while that between $M_{3}$ and $M_{4}$ goes from $\infty$ to $R_{c}$ in the transition state. In the transition state, the force constant $F_{b s}$ is modified and combined with that of the newly formed bond between $M_{3}$ and $M_{4}$ to produce two force constants $F_{\rho}$ and $F_{\sigma} . F_{\sigma}$ corresponds to the stable symmetric stretch and $F_{\rho}$ to the unstable asymmetric stretch. In cases $I V a$ and $V$, the bending force constant $F_{\star 2}$ becomes $F_{\star 2}$ in the complex. The newly formed bond angle made by $M_{2}, M_{3}$, and $M_{4}$ leads to the force constant $F_{* 3}$ in all cases. Finally, in cases $\mathrm{IVb}$ and $\mathrm{V}$, we also have an additional bending force constant $F_{* 4}$ which goes to $F_{\psi 4}$ in the second product. The force constants associated with the out-of-plane bends are not shown since they are the same as the in-plane constants.

The way I have chosen to assign values to the mass points is somewhat arbitrary and is best explained by an example. Consider the reaction

\section{Species}

$$
\begin{aligned}
& \mathrm{CH}_{3}-\mathrm{CH}_{2}-\mathrm{H}+\mathrm{CH}_{3}-\mathrm{CH}_{3}-\mathrm{CH}_{2} \cdot \cdot \mathrm{H} \cdot \bullet \mathrm{CH}_{3}-\mathrm{CH}_{3}-\mathrm{CH}_{2} \cdot+\mathrm{H} \cdot \mathrm{CH}_{3} \\
& \mathrm{~A} \quad \mathrm{~B} \quad \mathrm{C} \quad \mathrm{D}
\end{aligned}
$$

which is the abstraction of hydrogen from ethane by methyl radicals. The masses are assigned according to the following rules:

1) The mass of the transferred $\mathrm{H}$ is always assigned to $M_{3}$; therefore $M_{3}=1.008$ atomic mass units (a.m.u.).

2) The mass of the atom joined to the transferred $\mathrm{H}$ in reactant $A$ is assigned to $M_{2}$; in this case $M_{2}=$ 12.011 a.m.u.

3) The masses of all the remaining atoms in $\mathrm{A}$ are added and assigned to $M_{1}$; thus in this example $M_{1}=$ 17.051 a.m.u.

4) The mass of the atom joined to the transferred $\mathrm{H}$ in the product $\mathrm{D}$ is assigned to $M_{4}$; here $M_{4}=12.011$ a.m.u.

5) The masses of all the remaining atoms in $D$ are added and assigned to $M_{5}$; thus $M_{5}=3.024$ a.m.u. in this example.

Different models for the transition state, and different ways of arranging the masses in linear models have been explored in a limited way by Johnston [4] and by Sharp \& Johnston [5]. They did find significant differences between various options. Presumably, complete vibrational analyses of the reactant and complex would yield more accurate rate constants than the linear models outlined above. Unfortunately, complete analyses are extremely complex even for fairly small molecules, and the ability to program the calculations 
III

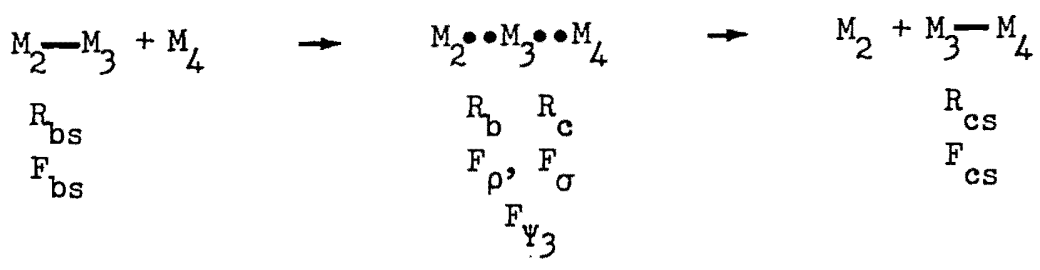

IVa

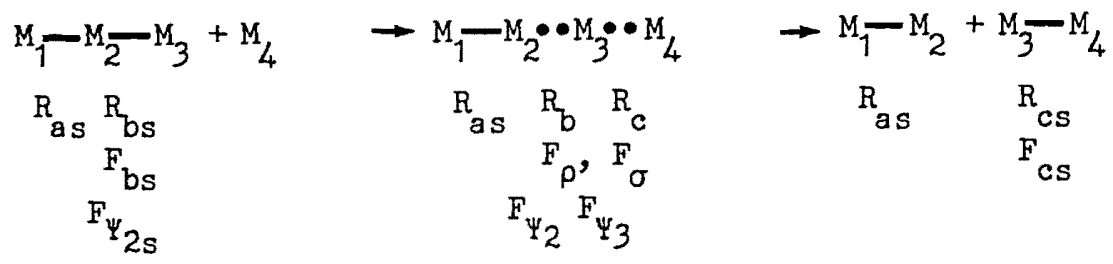

$\mathrm{IVb}$

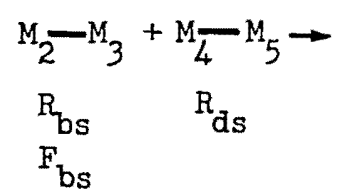

$$
\begin{gathered}
M_{2} \bullet M_{3} \bullet M_{4}-M_{5} \rightarrow \\
R_{b} R_{c} R_{d s} \\
F_{p_{F}}, F_{\sigma} F_{F_{4}} F_{\Psi_{4}}
\end{gathered}
$$$$
M_{2}+M_{3}-M_{4}-M_{5}
$$$$
\mathrm{R}_{\mathrm{cs}} \mathrm{R}_{\mathrm{ds}}
$$

$$
\begin{aligned}
& M_{1}-M_{2}-M_{3}+M-M_{5} \rightarrow M-M_{2} \cdot M_{3} \cdot M-M_{5} \rightarrow M_{1}-M_{2}+M_{3}-M_{4}-M_{5} \\
& \mathrm{R}_{\text {as }} \underset{\mathrm{F}_{\mathrm{bs}}}{\mathrm{R}_{\mathrm{bs}}} \\
& \mathrm{R}_{\mathrm{ds}} \\
& R_{a s} \mathbb{R}_{F_{\rho}}, F_{\sigma} R_{d s} \\
& \text { Ras } \\
& \mathrm{F}_{\mathrm{cs}} \mathrm{R}_{\mathrm{ds}} \\
& \mathrm{F}_{\Psi_{2 \mathrm{~S}}} \\
& \mathrm{~F}_{\Psi_{2}} \mathrm{~F}_{\Psi_{3}} \mathrm{~F}_{\Psi_{4}}
\end{aligned}
$$

Ficure Ib. Reaction cases to be used in BEBO calculations. All transition states shown here are linear. Masses are denoted by $M$, internuclear distances by $R$, and force constants by $F$. The subscript $s$ denotes bond distances and force constants in the stable reactants and products.

in a general manner would be lost by such an approach. Also, it is unlikely that all of the force constant values required would be available for a complete analysis. In view of the crudity of the rest of the calculation, it is unnecessary to strive for high accuracy in the vibrational analysis. Intuitively, one expects that the major features of these reactions are controlled by the nature of the atoms adjacent to the $\mathrm{H}$ atom being transferred, with the effects from the remainder of the molecule appearing in the bond energy values. If this is the case, then the linear models should at least be able to match trends within homologous series.

So far, we have seen in this section that evaluation of rate constants by the use of eq (3), based on the linear models shown in figure $\mathrm{lb}$, requires a knowledge of the potential energy $\mathrm{V}^{*}$ of the complex, two stretching force constants, and from one to three bending force constants. The potential energy of all of these linear models could, if it were known, be shown on a 2-dimensional contour diagram like that shown in figure 2 where the independent variables are the bond distances $R_{b}$ and $R_{c}$. The required value of the potential energy $V$ is that at the saddle point position shown by the asterisk. For a region close to the saddle point, 


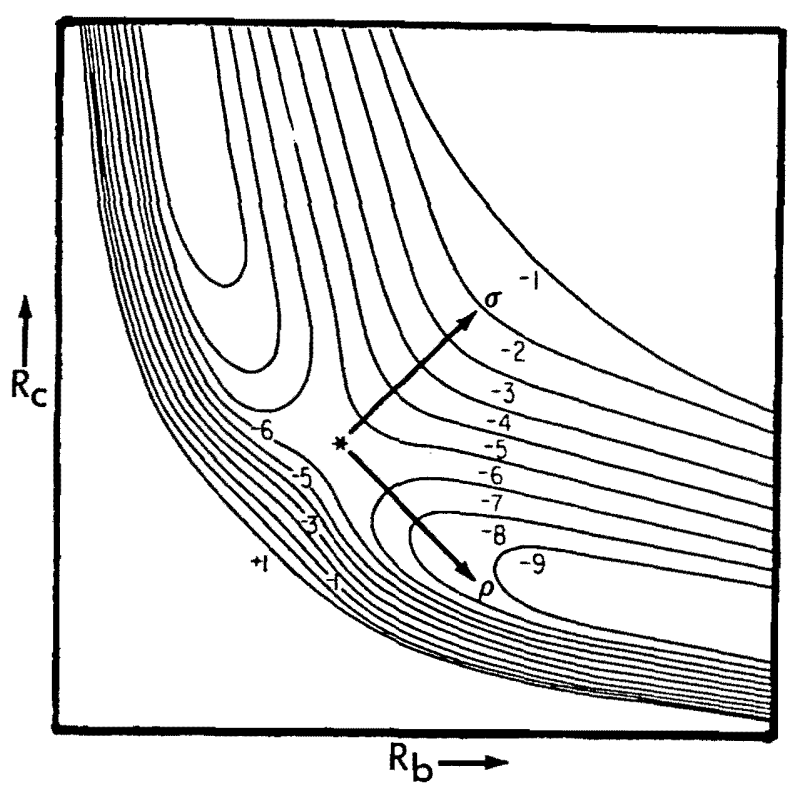

Ficure 2. Typical potential energy diagram for $\mathrm{H}$ atom exchange reaction. The position of the saddle point is shown by the asterisk. The direction $\mathrm{Q}$ is that in which the potential energy decreases most rapidly. The direction $\sigma$ is perpendicular to the $\mathrm{Q}$ direction.

it is customary to assume that the first derivatives of $\mathrm{V}$ with respect to $R_{b}$ and $R_{c}$ are negligible, and that the potential energy can be approximated by a power series containing only quadratic terms. Thus, for small displacements from the saddle point, we have

$$
2 \delta V \approx F_{11}\left(\delta R_{b}\right)^{2}+2 F_{12}\left(\delta R_{b}\right)\left(\delta R_{c}\right)+F_{22}\left(\delta R_{c}\right)^{2}
$$

where $F_{11}=\frac{\partial^{2} V}{\partial R_{b}^{2}}, F_{12}=F_{21}=\frac{\partial^{2} V}{\partial R_{b} \partial R_{c}^{\prime}}, F_{22}=\frac{\partial^{2} V}{\partial R_{c}^{2}}$.

These derivatives are evaluated at the saddle point, and are, by definition, the stretching force constants of the complex. In matrix notation, this equation is

$$
2 \delta V \approx(\delta \mathbf{R})+\mathbf{F}_{r}(\delta \mathbf{R})
$$

where $\mathbf{F}_{r}=\left[\begin{array}{ll}F_{11} & F_{12} \\ F_{21} & F_{22}\end{array}\right]$ and $\delta \mathbf{R}=\left[\begin{array}{l}\delta R_{b} \\ \delta R_{c}\end{array}\right]$

This is the force constant matrix that will be used to calculate the vibrational stretching frequencies.

Starting at the saddle point, suppose we move in the direction in which $V$ decreases most rapidly; call this the $\varrho$ direction, and let $\sigma$ denote the direction perpendicular to $\varrho$. These directions define a rotated set of cartesian coordinates which we assume makes an angle $\alpha$ with the $R_{b}$ axis; (positive $\alpha$ is measured in the counter-clockwide direction). The transformation between the two sets of coordinates is given by the equation

$$
\mathbf{R}=\left[\begin{array}{l}
R_{b} \\
R_{c}
\end{array}\right]=\left[\begin{array}{rr}
\cos \alpha & -\sin \alpha \\
\sin \alpha & \cos \alpha
\end{array}\right]\left[\begin{array}{l}
\varrho \\
\sigma
\end{array}\right]=\mathbf{U P}
$$

the matrix $\mathrm{U}$ can now be used to express changes in $V$ at the saddle point in terms of changes in $\varrho$ and $\sigma$ instead of $R_{b}$ and $R_{c}$. Thus, eq $(5 \mathrm{~b})$ becomes

$$
2 \delta V \approx(\delta \mathbf{R}) \dagger \mathbf{F}_{r}(\delta \mathbf{R})=(\mathbf{U} \delta \mathbf{P}) \dagger \mathbf{F}_{r}(\mathbf{U} \delta \mathbf{P})=(\delta \mathbf{P}) \dagger\left(\mathbf{U} \dagger \mathbf{F}_{r} \mathbf{U}\right)(\delta \mathbf{P})
$$


The matrix $\mathbf{U}+\mathbf{F}, \mathbf{U}$ has the elements

$$
\begin{gathered}
(\mathbf{U}+\mathbf{F}, \mathbf{U})_{11}=F_{11} \cos ^{2} \alpha+2 F_{12} \cos \alpha \sin \alpha+F_{22} \sin ^{2} \alpha \\
(\mathbf{U}+\mathbf{F}, \mathbf{U})_{12}=\left(\mathbf{U}+F_{r}, \mathbf{U}\right)_{21}=\left(F_{22}-F_{11}\right) \cos \alpha \sin \alpha+F_{12}\left(\cos ^{2} \alpha-\sin ^{2} \alpha\right) \\
\left(\mathbf{U}+F_{r} \mathbf{U}\right)_{22}=F_{11} \sin ^{2} \alpha-2 F_{12} \cos \alpha \sin \alpha+F_{22} \cos ^{2} \alpha
\end{gathered}
$$

As we shall see in the next section, the BEBO method provides values for the second derivatives of $V$ (i.e., the force constants) in the $\varrho$ and $\sigma$ directions. This will allow us to evaluate the matrix $\mathbf{U}+\mathbf{F}, \mathbf{U}$. The stretching force constant matrix $\mathbf{F}_{r}$, can then be obtained by inverting the transformation given by eq (6).

In this section I have presented a formula (eq (3)) for the rate constant and outlined the factors required to evaluate it. The details of the BEBO method will be given next. It will provide values for $V^{*}$ and all of the necessary force constants, both the stretching and the bending ones.

\section{BEBO Method}

The BEBO method is based on the concept of bond order. In the reactants the bond b of figure la is said to have a bond order of unity, while in the products, its bond order is zero. The reverse of this situation holds for bond $c$. BEBO assumes that during the reaction, the total bond order of the two bonds is conserved; if $n$ is the order of bond $b$, and $m$ of bond $c$, then we have always $n+m=1$. This is the basic assumption of the method. One bond is breaking at the same time that the other is forming. To apply this conservation condition it is necessary to relate the energies and lengths of bonds $b$ and $c$ to their bond orders $n$ and $m$.

For the relationship between order and length, Pauling [6] proposed the formula

$$
R_{n}=R_{s}-\lambda \ln (n)
$$

where $R$, is the length of the bond which is considered to be representative of a single bond between the two elements of interest. The parameter $\lambda$ is taken to have the same value for all element pairs. A plot of bond length versus the logarithm of the bond order is shown in figure 3 for certain element pairs. The data were

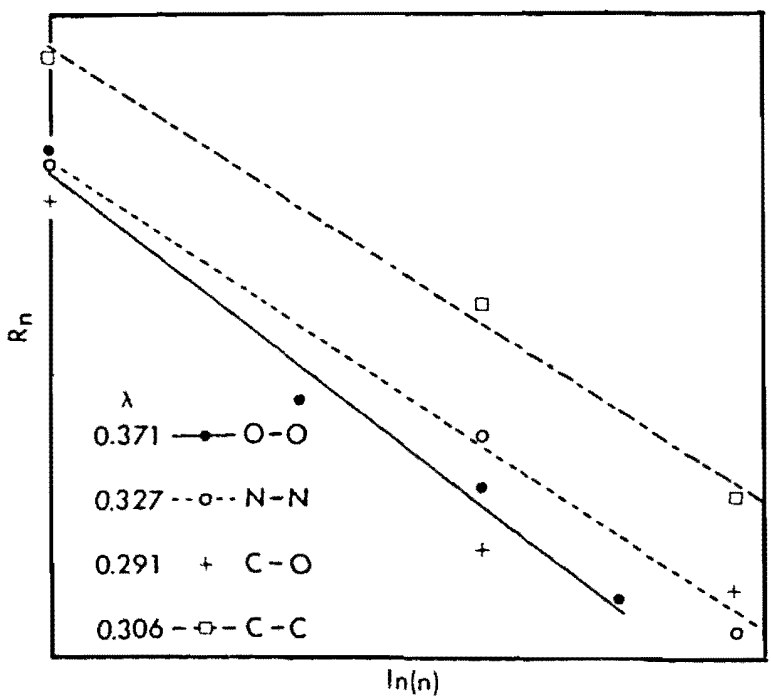

Figtre 3. Plot of $R_{n}=R, \lambda(n) n$, bond distance versus the natural loga* rithm of the bond order for selected element pairs. 
obtained from table 4-3 of Johnston's book [2]. Values of $\lambda$ obtained from least squares fits are given in the figure for the different bonds. The constancy of $\lambda$ does not seem particularly striking. Pauling chose a value of 0.26 for $\lambda$; he writes, "This equation, which is based upon the study of interatomic distances for nonresonating and resonating covalent bonds in simple non-metallic substances of known structure, is found to agree reasonably well with those data for metallic crystals which are suited to a check on its validity, and its use permits a penetrating analysis of the structure of metals and intermetallic compounds to be made. There is some evidence that the constant . . . varies with the kind of atom and with the type of bond; but the evidence is not sufficiently extensive to lead to the determination of the nature of this variation." Certainly Pauling's value doesn't appear to have been based very heavily on the data in figure 3 since none of these $\lambda$ values are close to 0.26 . Although 0.26 can hardly be construed as universal, it has nevertheless been the value used for most BEBO calculations. There appears to be no reason why a different value shouldn't be used if it gave better results.

Consider next the dependence of bond energy on bond order. Johnston [4] proposed the following relationship between the two quantities

$$
E_{n}=E_{s} n^{p}
$$

where $E_{s}$ is the bond energy of a single bond and is analogous to $R_{s}$ of eq (9). Note that this energy is the electronic dissociation energy of the bond in question; the zero point energy is not meant to be included in $E_{s}$. Plots of $\ln (\mathrm{E})$ versus $\ln (n)$ are shown in figure 4 for the same bonds used in figure 3 . The data are again from table 4-3 of Johnston [2]. We see that $p$ depends on the kind of atoms in the bond. If more than one bond type occurs for a pair of atoms, then it is possible to extract values for $p$ from plots like figure 4 provided we are not unduly bothered by a lack of linearity. When only a single bond type exists, then some other method must be devised. Actually, since we are interested in $E_{n}$ and $R_{n}$ for bond orders less than unity, even if multiple bonds were available for a plot like figure 4, some method of extrapolating to zero $n$ would be necessary. Johnston [2], inspired by Badger's rule for force constants, has devised a way. Let us first eliminate $\mathrm{n}$ between eqs (9) and (10); this yields

$$
\ln \left(E_{n} / E_{s}\right)=(p / \lambda)\left(R_{s}-R_{n}\right)
$$

This expression is analogous to Badger's rule (see Herschbach \& Laurie [7]), which is a universal empirical

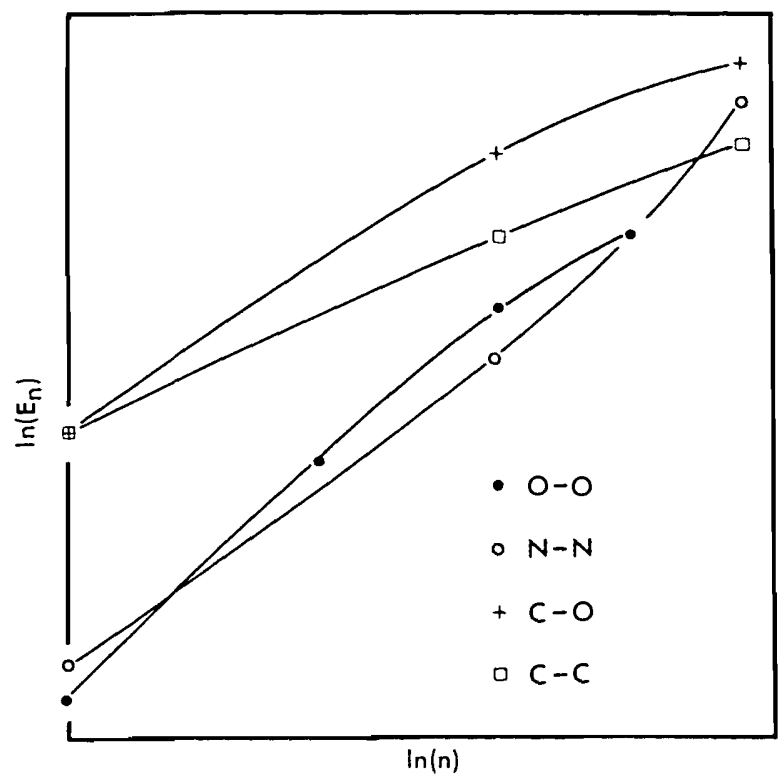

FIGURE 4. Plots of $\ln \left(E_{n}\right)$ versus $\ln (n)$ for certain bonds. 
relation having the form $r=a_{i j}-b_{i j} \log (f)$, where $r$ is the bond distance, $\mathrm{f}$ its force constant, $a_{i j}$ and $b_{i j}$ are constants, and $i$ and $j$ are the numbers of the rows in the periodic table in which the bound atoms are located. Johnston [8] found that plots of $\log (f)$ versus $r$ extrapolated very nicely to two-atom Lennard-Jones noble gas clusters. For clusters having Lennard-Jones parameters $\sigma$ and $\epsilon l k$, the "bond" distance is $2 \% \sigma$ and the "force" constant is $40.06(\epsilon / k) / \sigma^{2}$. He then examined plots of $\ln \left(E_{n}\right)$ versus $R_{n}$ to see if a comparable extrapolation would be possible. The results are shown in figure 5. The data are mostly from Johnston [2], tables 4-3 and 4-1. Values of $E_{n}$ and $R_{n}$ for the He-Ne cluster were taken from Gilliom [9]. The energies for the bonds examined in figures 3 and 4 are supposed to extrapolate to the Ne-Ne cluster. The lines shown were drawn to connect the corresponding single bonds with this cluster. Points corresponding to multiple bonds fall more or less in the general direction of these lines. The assumption made in BEBO is that such an extrapolation adequately represents the bond energies for $n<1$. Therefore, if we have a bond A-H, where $A$ is some atom in the first row of the periodic table connected to an $\mathrm{H}$ atom, and $R_{s}$ and $E_{s}$ are its bond length and energy, then if this bond were perturbed in some fashion so that its bond length were greater than $R_{s}$, then its bond energy would fall on the line drawn between the $\mathrm{A}-\mathrm{H}$ and $\mathrm{He}-\mathrm{Ne}$ points. Bonds involving atoms A from other rows of the periodic table will extrapolate to the appropriate rare gas-helium cluster. The slope of the line joining A-H to the cluster is, from eq (11), $-p / \lambda$. Since the value of $\lambda$ has been chosen, we have a way of calculating $\mathrm{p}$ for the A-H bond of interest. Formally, in this case,

$$
p=\frac{\lambda}{R_{s}-R_{\text {HeNe }}} \ln \left(E_{\text {HeNe }} / F_{s}\right)
$$

The parameter $\mathrm{p}$ thus depends on $\lambda$, the bond energy and internuclear distance of $\mathrm{A} \cdot \mathrm{H}$, and the interaction parameters for the appropriate rare gas cluster.

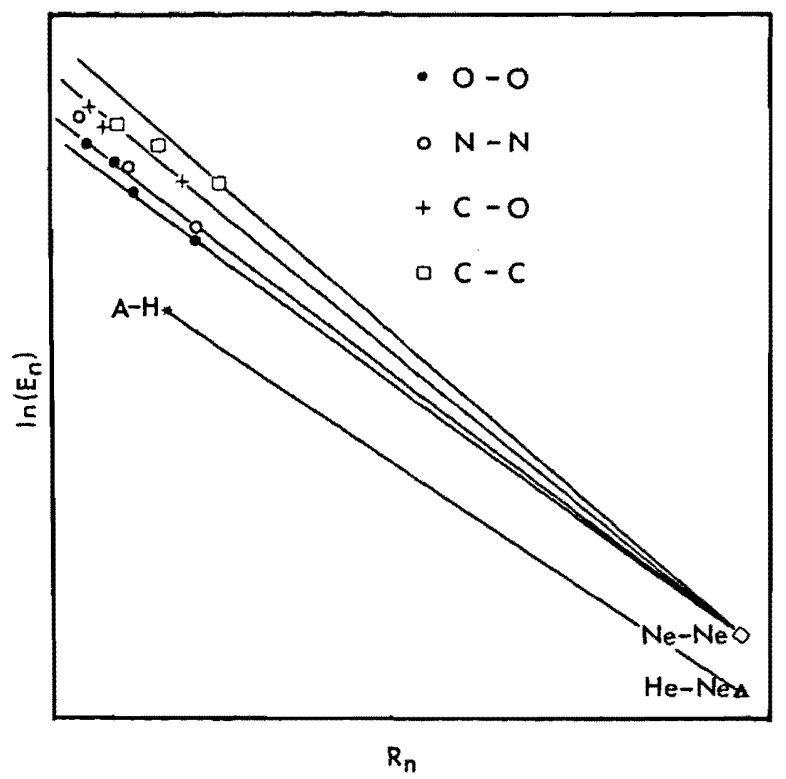

FIGURE 5. Extrapolation of bond energy to large bond distances. $A$ of $A \cdot H$ is an atom in the first row of the periodic table in this case.

We have now almost all of the information needed for the BEBO calculations. Consider a triatomic complex $\mathrm{A}-\mathrm{H}-\mathrm{B}$; there are three interactions; two between $\mathrm{H}$ and the atoms $\mathrm{A}$ and $\mathrm{B}$ considered above, and the interaction between $A$ and $B$ themselves. If $H$ is to form stable bonds its electron spin must be opposite each of the spins of $A$ and $B$. Consequently, $A$ and $B$ will have parallel spins and must repel each other. Johnston uses one half the value of the Sato [10] triplet function to represent this interaction. He uses the modified function because it more closely approximates the calculated $\mathrm{H}-\mathrm{H}$ triplet interaction. This function has the form 


$$
V_{s}=E_{t s} E(1+E)
$$

where $E=1 / 2 e^{-\beta \Delta R_{t}}, \Delta R_{t}=R_{t}-R_{t s}=R_{b}+R_{c}-R_{t s} . E_{t s}$ is the electronic dissociation energy, $R_{t s}$ the equilibrium internuclear distance, and $\beta$ the Morse parameter (see Herzberg [11] p. 101) of the ground state of the diatomic molecule made up of $A$ and $B$. Values of these paramaters for a number of such atom pairs are given in Table 11-1 of Johnston [2]. $\Delta R_{t}$ is the difference between the actual distance $R_{t}$ between $A$ and $B$ in the complex and the equilibrium distance $R_{t}$ it would have in the diatomic molecule. It is worth pointing out that many people use $E_{t s}$ as an adjustable parameter to fit the BEBO calculations to their experimental data. Other forms of the triplet function have been used and are discussed briefly in the Appendix. $V_{t}$ can be expressed as a function of $n$, the bond order of the $b$ bond, through the conservation condition $n+m=$ 1 , and through eq (9) which gives the distances $R_{b}$ and $R_{c}$ in terms of $n$ and $m$.

We are now able to give the BEBO expression for the energy of the complex in terms of the bond order $n$. The energy is assumed to be given by

$$
V(n)=E_{b s}-E_{b s} n^{p}-E_{c s} m^{q}+V_{t}(n)=E_{b s}\left(1-n^{p}\right)-E_{c s}(1-n)^{q}+V_{t}(n)
$$

$E_{b s}$ and $E_{c s}$ are the single bond energies (electronic) for bonds b and c, and the parameters $p$ and $q$ are calculated from eq (12) for $b$ and $c$, respectively. When $n-1$, then $m \rightarrow 0, V_{t} \rightarrow 0$, and $V \rightarrow 0$, so that the energy is measured relative to the energy of the reactants. When $n \rightarrow 0$, then $m-1, V_{\mathrm{t}}-0$, and $V-E_{b s}-E_{c s}$ which is the difference in the bond energies. BEBO assumes that the maximum value of $V$ in the range $1 \geq n \geq 0$ is the desired potential energy of the saddle point. This value $V^{*}$, is obtained by substituting into eq (14) that value of $\mathrm{n}$ which makes $d V / d n=0$. In what follows, all quantities are considered to be evaluated at the saddle point.

Next, we must determine the stretching force constants in the $\varrho$ and $\sigma$ directions shown in figure 3 . Equation (14) does not give the complete potential surface, but only that portion lying along the line of constant total bond order. BEBO assumes that at the saddle point, this path of constant bond order lies in the $\varrho$ direction. This assumption will enable us to calculate the force constant $F_{\mathrm{e}} \equiv \partial^{2} V / \partial \mathrm{Q}^{2}$ from the second derivative of $V$ with respect to n, which we get by differentiating eq (14).

From eq (9), we can calculate the changes produced in $R_{b}$ and $R_{c}$ when $n$ is changed. In vector notation these are

$$
\delta \mathbf{R}=\left[\begin{array}{l}
\delta R_{b} \\
\delta R_{c}
\end{array}\right]=-\lambda\left[\begin{array}{c}
1 / n \\
-1 / m
\end{array}\right] \delta n ; m=1-n
$$

Because a change in $\mathrm{n}$ for constant total bond order is supposed to produce a move in the $\varrho$ direction, the slope of a line in this direction can be gotten from eq (15). It is

$$
\delta R_{c} l \delta R_{b}=-n / m=\tan \alpha
$$

where $\alpha$ is the angle which $\varrho$ makes with the $R_{b}$ axis as discussed earlier. From eq (15) we can show that

$$
\cos \alpha=m / \sqrt{ }\left(n^{2}+m^{2}\right) ; \sin \alpha=-n / \sqrt{ }\left(n^{2}+m^{2}\right)
$$

The matrix $U$ defined in eq (6) can now be written in terms of $n$ and $m$.

$$
\mathrm{U}=\frac{1}{\sqrt{\left(n^{2}+m^{2}\right)}}\left[\begin{array}{c}
m n \\
-n m
\end{array}\right]
$$

By means of eq (6), $\delta \mathbf{R}$ can be expressed in terms of $\delta \mathbf{P}$; i.e., $\delta \varrho$ and $\delta \sigma$. Combining the differential form of eq (6) with eq (15), we get

$$
\mathbf{U} \delta \mathbf{P}=-\lambda\left[\begin{array}{c}
1 / n \\
-1 / m
\end{array}\right] \delta n
$$


Solving for $\delta \mathbf{P}$ gives

$$
\begin{gathered}
\delta \mathbf{P}=\left[\begin{array}{l}
\delta \varrho \\
\delta \varrho
\end{array}\right]=-\lambda \mathbf{U}^{-1}\left[\begin{array}{c}
1 / n \\
-1 / m
\end{array}\right] \delta n= \\
-\frac{\lambda}{\sqrt{\left(n^{2}+m^{2}\right)}}\left[\begin{array}{cc}
m-n \\
n & m
\end{array}\right]\left[\begin{array}{c}
1 / n \\
-1 / m
\end{array}\right] \delta n=-\lambda \sqrt{ }\left(n^{2}+m^{2}\right)\left[\begin{array}{c}
1 / n m \\
0
\end{array}\right] \delta n
\end{gathered}
$$

As expected, $\sigma$ does not change when $n$ changes. From eq $(20)$, we have for the derivative of $n$ with respect to e

$$
\frac{\delta n}{\delta \varrho}-\frac{d n}{d \varrho}=-\frac{1}{\lambda} \frac{n m}{\sqrt{ }\left(n^{2}+m^{2}\right)}
$$

The second derivative of $V$ with respect to $\varrho$ is obtained from the sequence

$$
\begin{gathered}
\frac{d V}{d \varrho}=\frac{d V}{d n} \frac{d n}{d \varrho} \\
\frac{d^{2} V}{d \varrho^{2}}=\frac{d^{2} V}{d n^{2}}\left(\frac{d n}{d \varrho}\right)^{2}+\frac{d V}{d n} \frac{d^{2} n}{d \varrho^{2}}
\end{gathered}
$$

Since $d V / d n=0$ at the saddle point, we have

$$
F_{\rho}=\frac{d^{2} V}{d \varrho^{2}}=\frac{d^{2} V}{d n^{2}} \frac{n^{2} m^{2}}{\lambda^{2}\left(n^{2}+m^{2}\right)}
$$

This gives one of the stretching force constants.

In the $\sigma$ direction, the stretching motion is assumed to be that of a normal molecule. Thus Badger's rule should be applicable. This says that the bond distance is proportional to the logarithm of the force constant, while eq (9) says that the bond distance is proportional to the logarithm of the bond order. Therefore, the force constant should be proportional to the bond order. We assume that

$$
F_{b}=F_{b s} n \text {, and } F_{c}=F_{c s} m
$$

where $F_{b s}$ and $F_{c}$ are single bond force constants. Consider the change in $V$ when $R_{b}$ and $R_{c}$ are changed by motion in the $\sigma$ direction. This is assumed to be given by

$$
2(\delta V)_{o}=F_{b s} n\left(\delta R_{b}\right)_{o}^{2}+F_{c s} m\left(\delta R_{c}\right)_{\sigma}^{2}+\frac{\partial^{2} V_{t}}{\partial R_{t}^{2}}\left(\delta R_{t}\right)_{\sigma}^{2}=F_{o}(\delta \sigma)^{2}
$$

To evaluate $F_{s}$, we must express $\left(\delta R_{b}\right)_{\sigma}^{2}\left(\delta R_{c}\right)_{\sigma}^{2}$, and $\left(\delta R_{t}\right)_{\sigma}^{2}$ in terms of $(\delta \sigma)^{2}$. From Eqs (6) and (18), we have

For $\delta \varrho=0$,

$$
\delta \mathbf{R}=\left[\begin{array}{l}
\delta R_{b} \\
\delta R_{c}
\end{array}\right]=\frac{1}{\sqrt{\left(n^{2}+m^{2}\right)}} \quad\left[\begin{array}{cc}
m & n \\
-n & m
\end{array}\right]\left[\begin{array}{l}
\delta_{\rho} \\
\delta_{\sigma}
\end{array}\right]
$$

$$
\begin{gathered}
\left(\delta R_{b}\right)_{\sigma}=n \delta \sigma / \sqrt{ }\left(n^{2}+m^{2}\right), \quad\left(\delta R_{c}\right)_{\sigma}=m \delta \sigma / \sqrt{ }\left(n^{2}+m^{2}\right) \\
\left(\delta R_{z}\right)_{\sigma}=\left(\delta R_{b}\right)_{\sigma}+\left(\delta R_{c}\right)_{\sigma}=(n+m) \delta \sigma / \sqrt{ }\left(n^{2}+m^{2}\right)=\delta \sigma / \sqrt{ }\left(n^{2}+m^{2}\right) .
\end{gathered}
$$

Therefore,

$$
2(\delta V)_{\sigma}=\left(F_{b s} n^{3}+F_{c s} m^{3}+\frac{\partial^{2} V_{t}}{\partial R_{t}^{2}}\right)(\delta \sigma)^{2} /\left(n^{2}+m^{2}\right)
$$


Comparing this with eq (24) gives

$$
F_{\sigma}=\frac{F_{b s} n^{3}+F_{c s} m^{3}+\partial^{2} V_{t} / \partial R_{t}^{2}}{n^{2}+m^{2}} .
$$

The method assumes that if $V$ is expanded at the saddle point in terms of $\varrho$ and $\sigma$ then there is no cross term; i.e., $\partial^{2} V / \partial \varrho \partial \sigma$ is assumed to be zero. Thus, we have

$$
2 \delta V=(\delta \mathbf{P}) \dagger\left[\begin{array}{ll}
F_{\mathrm{Q}} & 0 \\
0 & F_{\sigma}
\end{array}\right](\delta \mathbf{P})
$$

The use of eq (7) shows that

$$
\mathbf{U}+\mathbf{F}_{r} \mathbf{U}=\left[\begin{array}{cc}
F_{\mathrm{e}} & 0 \\
0 & F_{\sigma}
\end{array}\right]
$$

Inverting this equation gives

$$
\mathbf{F}_{r}=(\mathbf{U} \dagger)^{-1}\left[\begin{array}{cc}
F_{Q} & 0 \\
0 & F_{\sigma}
\end{array}\right] \mathbf{U}^{-1}=\mathbf{U}\left[\begin{array}{ll}
F_{Q} & 0 \\
0 & F_{\sigma}
\end{array}\right] \mathbf{U} \dagger
$$

where use has been made of the fact that $\mathbf{U}^{-1}=\mathbf{U}$. Substituting eq (18) into (29) gives the desired stretching force constant matrix.

$$
\begin{aligned}
\mathbf{F}_{r}=\frac{1}{n^{2}+m^{2}}\left[\begin{array}{cc}
m & n \\
-n & m
\end{array}\right]\left[\begin{array}{cc}
F_{\mathrm{e}} & 0 \\
0 & F_{\sigma}
\end{array}\right]\left[\begin{array}{cc}
m & -n \\
n & m
\end{array}\right] \\
=\frac{1}{n^{2}+m^{2}}\left[\begin{array}{l}
F_{\mathrm{e}} m^{2}+F_{\mathrm{o}} n^{2},-F_{\mathrm{e}} m n+F_{\mathrm{o}} m n \\
-F_{\mathrm{e}} m n+F_{\mathrm{o}} m n, F_{\mathrm{e}} n^{2}+F_{\mathrm{o}} m^{2}
\end{array}\right]=\left[\begin{array}{ll}
F_{11} & F_{12} \\
F_{21} & F_{22}
\end{array}\right]
\end{aligned}
$$

To complete the discussion of the BEBO method the bending force constants will now be evaluated. Consider first the one involving $M_{3}$ as the center mass. This will be $F_{\psi_{3}}$ and appears in all of the transition states shown in figure 2. It is defined as the second partial derivative of $V$ with respect to the angle made by the bonds $b$ and $c$, with the bond lengths $R_{b}$ and $R_{c}$ held fixed. At equilibrium, this angle is $180^{\circ}$ for our transition state models. The geometry, when the angle is less than $180^{\circ}$ is shown in figure 6 . To get $F_{\downarrow_{3}}$, we differentiate $V$ twice,

$$
\begin{gathered}
\left(\frac{\partial V}{\partial \phi}\right)_{R_{b}, R_{c}}=\frac{\partial V_{t}}{\partial \phi}=\frac{\partial V_{t}}{\partial R_{t}} \frac{\partial R_{t}}{\partial \phi} \\
\left(\frac{\partial^{2} V}{\partial \phi^{2}}\right)_{R_{b}, R_{c}}=\frac{\partial^{2} V_{t}}{\partial \phi^{2}}=\frac{\partial^{2} V_{t}}{\partial R_{t}^{2}}\left(\frac{\partial R_{t}}{\partial \phi}\right)^{2}+\frac{\partial V_{t}}{\partial R_{t}} \frac{\partial^{2} R_{t}}{\partial \phi^{2}}
\end{gathered}
$$

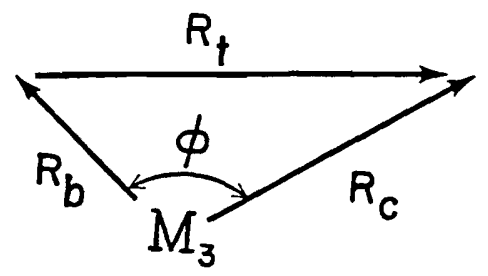

FIGURE 6. Defirition of center bond angle.

The derivatives of $V_{\mathrm{t}}$ with respect to $R_{\mathrm{t}}$ can be gotten from eq (13). The dependence of $R_{\mathrm{t}}$ on $\phi$ can be determined from the following vector relationships, 


$$
\begin{gathered}
\mathbf{R}_{b} \cdot \mathbf{R}_{c}=R_{b} R_{c} \cos \phi \\
\mathbf{R}_{t}=\mathbf{R}_{c}-\mathbf{R}_{b} \\
R_{t}^{2}=\mathbf{R}_{t} \cdot \mathbf{R}_{\mathrm{t}}=R_{b}^{2}+R_{c}^{2}-2 R_{b} R_{c} \cos \phi \\
\frac{\partial R_{t}}{\partial \phi}=\frac{R_{b} R_{c}}{R_{t}} \sin \phi-0 \text { for } \phi=180^{\circ} \\
\frac{\partial^{2} R_{t}}{\partial \phi^{2}}=\frac{R_{b} R_{c} \cos \phi}{R_{t}}-\frac{R_{b}^{2} R_{c}^{2}}{R_{t}^{2}} \sin ^{2} \phi--\frac{R_{b} R_{c}}{R_{t}} \text { for } \phi=180^{\circ}
\end{gathered}
$$

Thus,

$$
F_{v_{3}}=-\frac{\partial V_{\mathrm{s}}}{\partial R_{t}} \frac{R_{b} R_{c}}{R_{t}}=-\frac{\partial V_{\mathrm{t}}}{\partial R_{\mathrm{t}}} \frac{R_{b} R_{\mathrm{c}}}{R_{b}+R_{\mathrm{c}}}
$$

The other two bending force constants $F_{v_{2}}$ and $F_{v_{3}}$ are assumed to obey Badger's rule. We assume

$$
F_{\psi_{2}}=F_{\psi_{28}} n \text {, and } F_{\psi_{4}}=F_{\psi_{4} s} m
$$

This concludes the BEBO part of the calculation. It has provided us with the potential energy $V^{*}$ of the saddle point, the stretching force constants $F_{11}, F_{22}$, and $F_{12}$ and the bending force constants $F_{\psi_{2}}, F_{\psi_{3}}$, and $F_{\psi_{4}}$. In the next section we shall use these force constants to carry out a frequency analysis for each of the transition state models shown in figure 2.

\subsection{Vibrational Analysis}

As we have seen in the force constant derivations, the potential energy $\mathrm{V}$ of the most general 5 mass point complex can be considered to depend on the variables $R_{a}, R_{b}, R_{c}, R_{d}, \Psi_{2}, \Psi_{3}, \Psi_{4}, \Psi_{2}^{\prime}, \Psi_{3}^{\prime}$, and $\Psi_{4}^{\prime}$. These are called the internal coordinates. Because our model is linear, $V$ increases when any of the angles departs from $180^{\circ}$. Since we assume $a$ and $d$ to be rigid, $R_{a}$ and $R_{d}$ need not be included in the list of variables. For the time being, however, they will be included in the analysis. Let $\mathbf{F}$ be the complete force constant matrix for the complex. We have

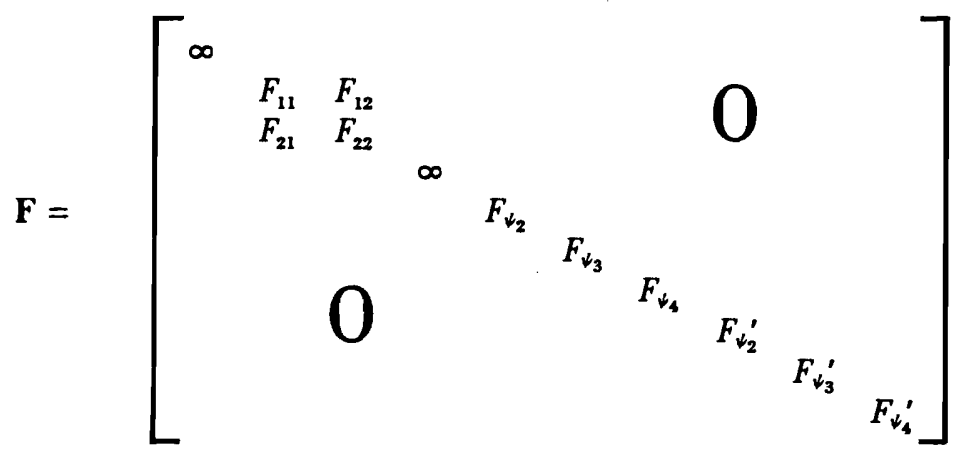

The two infinite force constants come from the use of rigid bonds for $a$ and $d$. Let $\mathbf{S}$ be the (column) vector which denotes small changes in the saddle point values of the variables.

$$
\mathrm{S} \dagger=\left[\delta R_{a}, \delta R_{b}, \delta R_{c}, \delta R_{d}, \delta \Psi_{2}, \delta \Psi_{3}, \delta \Psi_{4}, \delta \Psi_{2}^{\prime} \delta \Psi_{3}^{\prime} \delta \Psi_{4}^{\prime}\right]
$$

The potential energy is assumed to be given by

$$
V-V^{*}=1 / 2 \dot{\mathbf{S}}+\mathbf{F} \dot{\mathbf{S}}
$$


Suppose there exists a matrix $\mathbf{G}$, such that the kinetic energy in terms of the internal coordinates is

$$
T=1 / 2 \dot{\mathbf{S}}+\mathbf{G}^{-1} \dot{\mathbf{S}}
$$

Consider a new set of coordinates $\mathbf{Q}$, the so-called normal coordinates, related to $\mathbf{S}$ by the linear transformation

$$
\mathbf{S}=\mathbf{L Q}
$$

such that

$$
\begin{gathered}
V-V^{*}=1 / 2 \mathbf{Q} \dagger \Lambda \mathbf{Q}=\Delta V \\
T=1 / 2 \dot{\mathbf{Q}} \dagger \mathbf{E} \dot{\mathbf{Q}}
\end{gathered}
$$

where $\Lambda$ is a diagonal matrix having elements $\lambda_{i}$, and $\mathbf{E}$ is the identity matrix. In this coordinate system there are no cross terms in $V$ and $T$.

Let $Q_{i}$ denote the $i$ 'th normal coordinate. The Lagrangian equations of motion for the system are

$$
\begin{gathered}
\frac{d}{d t}\left(\frac{\partial L}{\partial \dot{Q}_{i}}\right)-\frac{\partial L}{\partial Q_{i}}=0 \\
\text { where } L=T-\Delta V=1 / 2[\dot{\mathbf{Q}}+\mathbf{E} \dot{\mathbf{Q}}-\mathbf{Q}+\Lambda \mathbf{Q}]=1 / 2\left[\sum_{j} \dot{Q}_{j}^{2}-\sum_{J} \lambda_{j} Q_{j}^{2}\right], \\
\frac{\partial L}{\partial \dot{Q}_{i}}=\dot{Q}_{i}, \\
\frac{\partial L}{\partial Q_{i}}=-\lambda_{i} Q_{i} .
\end{gathered}
$$

Therefore

$$
\ddot{Q}_{i}+\lambda_{i} Q_{i}=0
$$

The solutions of this equation are

$$
Q_{i}=Q_{i} \cos \left(\lambda_{i}^{1 / 2} t+\epsilon_{i}\right)
$$

Thus the $\lambda_{i}^{1 / 2}=2 \pi \nu_{i}$ are the frequencies of the vibrations of the $Q_{i}$ coordinates. These are called the normal mode vibrations.

Solving eq (39) for $\mathbf{Q}$, and substituting into (40) and (41) yields

$$
\begin{aligned}
V-V^{*} & =1 / 2\left(\mathbf{L}^{-1} \mathbf{S}\right) \dagger \Lambda\left(\mathbf{L}^{-1} \mathbf{S}\right)=1 / 2 \mathbf{S} \dagger\left(\mathbf{L}^{-1}\right) \dagger \Lambda\left(\mathbf{L}^{-1}\right) \mathbf{S} \\
T & =1 / 2\left(\mathbf{L}^{-1} \dot{\mathbf{S}}\right) \dagger \mathbf{E}\left(\mathbf{L}^{-1} \dot{\mathbf{S}}\right)=1 / 2 \dot{\mathbf{S}} \dagger\left(\mathbf{L}^{-1}\right) \dagger \mathbf{E}\left(\mathbf{L}^{-1}\right) \dot{\mathbf{S}}
\end{aligned}
$$

Comparison with eqs (37) and (38) yields

$$
\begin{gathered}
\mathbf{F}=\left(\mathbf{L}^{-1}\right) \dagger \Lambda\left(\mathbf{L}^{-1}\right) \\
\mathbf{L}+\mathbf{F} \mathbf{L}=\Lambda \\
\mathbf{G}^{-1}=\left(\mathbf{L}^{-1}\right) \dagger \mathbf{E}\left(\mathbf{L}^{-1}\right) \\
\mathbf{L}+\mathbf{G}^{-1} \mathbf{L}=\mathbf{E}
\end{gathered}
$$


Next, solve eq (53) for $\mathbf{L} \dagger=\mathbf{L}^{-1} \mathbf{G}$, substitute this into eq (51) and multiply by $\mathbf{L}$ on the left. This gives

$$
\mathbf{G F L}=\mathbf{H L}=\mathbf{L} \Lambda
$$

as the set of equations which determine the transformation $L$. Written out, eq (54) is

$$
\Sigma_{j}\left[H_{i j}-\delta_{i j} \lambda_{k}\right] L_{j k}=0
$$

This equation has solutions if the determinant

$$
\left|\mathbf{H}-\mathbf{E} \lambda_{k}\right|=0
$$

This is the so-called secular equation which must be solved to get the $\lambda_{k}$, the eigenvalues of $\mathbf{H}$ and thus the normal frequency values. Before doing this, it is first necessary to evaluate the matrix $\mathbf{G}$.

Equation (38) gives the kinetic energy in terms of the internal coordinates. As such, it does not include the kinetic energy of the center of mass or the rotational energy. We need to express the kinetic energy in terms of cartesian coordinates, transform the result to internal coordinates, and subtract out the center of mass and rotational energy. This will yield $\mathbf{G}^{-1}$. Let us begin by expressing the internal coordinates in terms of cartesian coordinates. Assume that the molecule lies along the $\mathrm{x}$ axis. A particular mass point $M_{i}$ will have coordinates $\left(x_{i}, y_{i}, z_{i}\right)$ where $y_{i}$ and $z_{i}$ are small and describe the departures of the molecule from linearity during bending vibrations. Because $y_{i}$ and $z_{i}$ are small, the bond distances can be expressed as functions of the $x_{i}$ only. Thus,

$$
\begin{aligned}
& R_{a}=x_{2}-x_{1} \\
& R_{b}=x_{3}-x_{2} \\
& R_{c}=x_{4}-x_{3} \\
& R_{d}=x_{5}-x_{4}
\end{aligned}
$$

Since there are 5 cartesian $x$ coordinates we need one more coordinate for the internal system. This is taken to be the $x$-component of the center of mass of the molecule multiplied by the total mass, and is defined by the equation,

$$
\begin{gathered}
M x=\sum_{i=1}^{5} M_{i} x_{i} \\
\text { where } M=\sum_{i=1}^{5} M_{i}
\end{gathered}
$$

In matrix form these equations are

$\tilde{\mathbf{R}}=\left[\begin{array}{l}\mathbf{R} \\ M x\end{array}\right]=\left[\begin{array}{l}R_{a} \\ R_{b} \\ \mathrm{R}_{c} \\ R_{d} \\ M x\end{array}\right]=\left[\begin{array}{rrrrr}-1 & 1 & 0 & 0 & 0 \\ 0 & -1 & 1 & 0 & 0 \\ 0 & 0 & -1 & 1 & 0 \\ 0 & 0 & 0 & -1 & 1 \\ M_{1} & M_{2} & M_{3} & M_{4} & M_{5}\end{array}\right]\left[\begin{array}{l}x_{1} \\ x_{2} \\ x_{3} \\ x_{4} \\ x_{5}\end{array}\right]=\mathbf{M X}$

Note that the vector $\mathbf{R}$ is basically that defined by eq (15). Here we have included $R_{\mathrm{a}}$ and $R_{\mathrm{d}}$.

We must next express the bond angles in terms of the cartesian coordinates. Consider $\Psi_{2}$, the angle formed by bonds $a$ and $b$. The geometry and notation for this angle are shown in figure 7. The two vectors along the bonds $a$ and $b$ are given by 


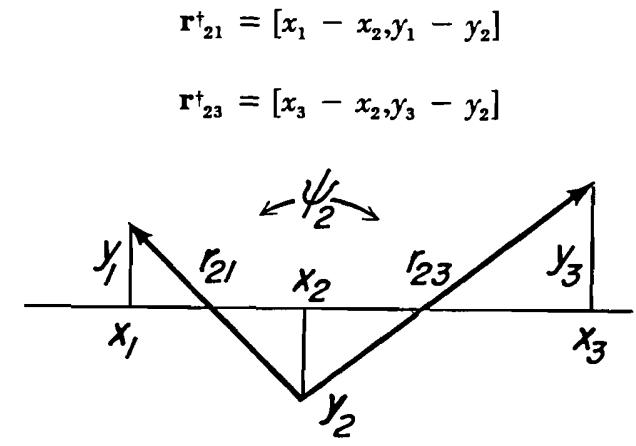

FIGURE 7. Geometry of the bond angle $\Psi_{2}$.

$\Psi_{2}$ is related to these by

$$
\mathbf{r}_{21} \cdot \mathbf{r}_{23}=r_{21} r_{23} \cos \Psi_{2}
$$

Substituting eq (70) into (71) gives

$$
-R_{a} R_{b}+\left(y_{1}-y_{2}\right)\left(y_{3}-y_{2}\right)=\left\{\left[R_{a}^{2}+\left(y_{1}-y_{2}\right)^{2}\right]\left[R_{b}^{2}+\left(y_{3}-y_{2}\right)^{2}\right]\right\}^{1 / 2} \cos \Psi_{2}
$$

Because the $y_{i}$ are small compared to $R_{a}$ and $R_{b}$ the radical can be expanded to give

$$
\begin{gathered}
-R_{a} R_{b}+\left(y_{1}-y_{2}\right)\left(y_{3}-y_{2}\right) \approx\left[R_{a} R_{b}+1 / 2\left(R_{b} / R_{a}\right)\left(y_{1}-y_{2}\right)^{2}+1 / 2\left(R_{a} / R_{b}\right)\left(y_{3}-y_{2}\right)^{2}\right] \\
\cdot \cos \Psi_{2}
\end{gathered}
$$

Let $\Psi_{2}=180^{\circ}+\delta \Psi_{2}$ where $\delta \Psi_{2}$ is small. Then

$$
\cos \Psi_{2}=-\cos \left(\delta \Psi_{2}\right) \approx-1+1 / 2\left(\delta \Psi_{2}\right)^{2}
$$

Substituting this into eq (73) and keeping terms through second order gives

$$
\begin{gathered}
\left(\delta \Psi_{2}\right)^{2}=\left(1 / R_{a}^{2}\right)\left(y_{1}-y_{2}\right)^{2}+\left(2 / R_{a} R_{b}\right)\left(y_{1}-y_{2}\right)\left(y_{3}-y_{2}\right)+\left(1 / R_{b}^{2}\right)\left(y_{3}-y_{2}\right)^{2} \\
\delta \Psi_{2}=-\left[\left(\gamma_{1}-y_{2}\right) / R_{a}+\left(y_{3}-y_{2}\right) / R_{b}\right]=-y_{1} / R_{a}+\left(1 / R_{a}+1 / R_{b}\right) y_{2}-y_{3} / R_{b}
\end{gathered}
$$

To see why the minus sign is needed, let $y_{1}=y_{2}=0$; then for $y_{3}>0, \Psi_{2}<180^{\circ}$, so that $\delta \Psi_{2}$ must be $<0$. There are analogous equations for the angles $\Psi_{3}$ and $\Psi_{4}$; there is also a set, identical in form, for the angles $\Psi_{i}^{\prime}$ in the x-z plane. These contain the $z_{i}$ rather than the $y_{i}$ coordinates. In these equations, the equilibrium values of $R_{a}, \ldots, R_{d}$ will be used.

The set of equations typified by eq (74) gives 3 equations in terms of the $5 y_{i}$ coordinates; two more are needed. We have one defining the $y$ coordinate of the center of mass, like eq (58), and another defining a quantity $\eta_{z}$, which is given by the equation

$$
\eta_{x}=\sum_{i=1}^{5} M_{i} x_{i}^{e} y_{i}
$$

$\eta_{z}$ is related to the $z$ component of the angular momentum $m_{z}$ by the relation

$$
m_{x}=\dot{\eta}_{x}
$$


The $x_{i}$ are the equilibrium $x_{i}$ values; these can be gotten relative to the center of mass component $x$, by inverting eq (60) and inserting equilibrium values for $R_{a}, \ldots, R_{d}$. In matrix form, these equations relating $y_{i}$ to the bond angles in the $x-y$ plane are,

$$
\bar{\Psi}=\left[\begin{array}{c}
\Psi \\
\eta_{x} \\
M y
\end{array}\right]=\left[\begin{array}{c}
\delta \psi_{2} \\
\delta \psi_{3} \\
\delta \psi_{4} \\
\eta_{x} \\
M y
\end{array}\right]=\left[\begin{array}{ccccc}
-\varrho_{a} & \varrho_{a}+\varrho_{b} & -\varrho_{b} & 0 & 0 \\
0 & -\varrho_{b} & \varrho_{b}+\varrho_{c} & -\varrho_{c} & 0 \\
0 & 0 & -\varrho_{c} & \varrho_{c}+\varrho_{d} & -\varrho_{d} \\
M_{1} x_{1}^{e} & M_{2} x_{2}^{e} & M_{3} x_{3}^{e} & M_{4} x_{4}^{e} & M_{5} x_{5}^{e} \\
M_{1} & M_{2} & M_{3} & M_{4} & M_{5}
\end{array}\right]\left[\begin{array}{l}
y_{1} \\
y_{2} \\
y_{3} \\
y_{4} \\
y_{5}
\end{array}\right]=\mathbf{A Y}
$$

where $\varrho_{a}, \ldots, \varrho_{d}$ are the reciprocals of the equilibrium values of $R_{a}, \ldots, R_{d}$. There is an analogous equation involving the $z_{i}$ coordinates.

Having obtained expressions (60) and (77) for the internal coordinates in terms of the cartesian coordinates, we can now invert these equations and insert them into the expression for the total kinetic energy which we shall call $\widetilde{T}$. Therefore

$$
\begin{aligned}
\widetilde{T} & =1 / 2 \dot{\mathbf{X}}+\mathbf{D}_{m} \dot{\mathbf{X}}+1 / 2 \dot{\mathbf{Y}}+\mathbf{D}_{m} \dot{\mathbf{Y}}+1 / 2 \dot{\mathbf{Z}} \dagger \mathbf{D}_{m} \dot{\mathbf{Z}} \\
& =1 / 2 \dot{\widetilde{\mathbf{R}}}+\left(\mathbf{M}^{-1}\right)+\mathbf{D}_{m}\left(\mathbf{M}^{-1}\right) \dot{\widetilde{\mathbf{R}}}+1 / 2 \dot{\bar{\Psi}}+\left(\mathbf{A}^{-1}\right)+\mathbf{D}_{m}\left(\mathbf{A}^{-1}\right) \dot{\widetilde{\Psi}}+z \text {-term } \\
& =1 / 2 \dot{\overrightarrow{\mathbf{R}}}+\widetilde{\mathbf{G}}_{r}^{-1} \dot{\overrightarrow{\mathbf{R}}}+1 / 2 \dot{\widetilde{\Psi}}+\widetilde{\mathbf{G}}_{\psi}^{-1} \dot{\bar{\Psi}}+z \text {-term } \\
& =1 / 2 T+1 / 2 M\left(\dot{x}^{2}+\dot{y}^{2}+\dot{z}^{2}\right)+1 / 2\left(m_{y}^{2}+m_{x}^{2}\right) / I \\
& =1 / 2 \dot{\mathbf{R}}+\mathbf{G}_{r}^{-1} \dot{\mathbf{R}}+1 / 2 \dot{\Psi}+\mathbf{G}_{\psi}^{-1} \dot{\Psi}+z \text {-term }+1 / 2 M\left(\dot{x}^{2}+\dot{y}^{2}+\dot{z}^{2}\right)+1 / 2\left(m_{y}^{2}+m_{z}^{2}\right) / I
\end{aligned}
$$

where $I=\sum_{i=1}^{5} M_{i} x_{i}^{i 2}$ is the moment of inertia, and

$$
\mathbf{D}_{m}=\left[\begin{array}{lllll}
M_{1} & & & 0 & \\
& M_{2} & & & \\
& & M_{3} & & \\
& & M_{4} & \\
& & & M_{5}
\end{array}\right]
$$

We can satisfy eq (78) by writing $\widetilde{\mathbf{G}}_{r}^{-1}$ and $\widetilde{\mathbf{G}}_{\psi}^{-1}$ in the partitioned forms

$$
\begin{gathered}
\widetilde{\mathbf{G}}_{r}^{-1}=\left[\begin{array}{ll}
\mathbf{G}_{r}^{-1} & 0 \\
0 & M^{-1}
\end{array}\right] \\
\tilde{\mathbf{G}}_{\psi}^{-1}=\left[\begin{array}{lll}
\mathbf{G}_{\psi}^{-1} & 0 & 0 \\
0 & I^{-1} & 0 \\
0 & 0 & M^{-1}
\end{array}\right]=\widetilde{\mathbf{G}}_{\psi^{\prime}}^{-1}
\end{gathered}
$$

We can get $\mathbf{G}_{\mathbf{r}}$ and $\mathbf{G}_{\downarrow}$ simply by inverting $\widetilde{\mathbf{G}}_{\mathbf{r}}^{-1}$ and $\widetilde{\mathbf{G}}_{\psi}^{-1}$.

This gives

$$
\begin{gathered}
\widetilde{\mathbf{G}}_{r}=\left[\begin{array}{ll}
\mathbf{G}_{r} & \overline{0} \\
0 & M
\end{array}\right]=\mathbf{M D}_{m}^{-1} \mathbf{M} \dagger \\
\widetilde{\mathbf{G}}_{\psi}=\left[\begin{array}{lll}
\mathbf{G}_{\psi} & 0 & 0 \\
0 & \mathrm{I} & 0 \\
0 & 0 & M
\end{array}\right]=\mathbf{A D}_{m}^{-1} \mathbf{A} \dagger
\end{gathered}
$$


Since $\mathbf{D}_{m}$ is diagonal its inverse is easily evaluated and we therefore require only matrix multiplications to get $\mathbf{G}_{r}$ and $\mathbf{G}_{\downarrow}$.

The complete $\mathbf{G}$ matrix for the internal coordinates in partitioned form is

$$
\mathbf{G}=\left[\begin{array}{lll}
\mathbf{G}_{\boldsymbol{r}} & 0 & 0 \\
0 & \mathbf{G}_{\boldsymbol{\psi}} & 0 \\
0 & 0 & \mathbf{G}_{\boldsymbol{\nu}^{\prime}}
\end{array}\right]
$$

In partitioned form, the complete force constant matrix, eq (35), is

$$
\mathbf{F}=\left[\begin{array}{lll}
\mathbf{F}_{r} & 0 & 0 \\
0 & \mathbf{F}_{\downarrow} & 0 \\
0 & 0 & \mathbf{F}_{\downarrow^{\prime}}
\end{array}\right]
$$

Note that $\mathbf{F}$, here is like eq (30), but contains the two infinite force constants corresponding to the rigid a and $\mathbf{d}$ bonds. The matrix $\mathbf{H}$ in partitioned form is

$$
\mathbf{H}=\left[\begin{array}{lll}
\mathbf{G}_{\boldsymbol{r}} \mathbf{F}_{\boldsymbol{r}} & 0 & 0 \\
0 & \mathbf{G}_{\psi^{\prime}} \mathbf{F}_{\psi} & 0 \\
0 & 0 & \mathbf{G}_{\psi^{\prime}} \mathbf{F}_{\psi^{\prime}}
\end{array}\right]=\left[\begin{array}{lll}
\mathbf{H} & 0 & 0 \\
0 & \mathbf{H}_{\psi} & 0 \\
0 & 0 & \mathbf{H}_{\psi^{\prime}}
\end{array}\right]
$$

Because $\mathbf{H}$ factors in this way, we can set up separate secular equations for the stretching and bending modes. Note that $\mathbf{H}$ is normally unsymmetric.

Before solving the secular equations, let us write down explicit expressions for $\mathbf{G}_{\boldsymbol{r}}$ and $\mathbf{G}_{\psi}$. The direct evaluation of $\widetilde{\mathbf{G}}_{\boldsymbol{r}}$ from eq (81) yields

$$
\widetilde{\mathbf{G}}_{r}=\left[\begin{array}{ccccc}
\mu_{1}+\mu_{2} & -\mu_{2} & 0 & 0 & 0 \\
-\mu_{2} & \mu_{2}+\mu_{3} & -\mu_{3} & 0 & 0 \\
0 & -\mu_{3} & \mu_{3}+\mu_{4} & -\mu_{4} & 0 \\
0 & 0 & -\mu_{4} & \mu_{4}+\mu_{5} & 0 \\
0 & 0 & 0 & 0 & M
\end{array}\right]
$$

where the $\mu_{i}$ are the reciprocals of the masses $M_{i}$. Comparison of this equation with eq (81) yields $\mathbf{G}_{r}$. Because we are treating the $a$ and $d$ bonds as rigid, the stretching part of the problems is equivalent to a 3 mass point system where the first mass is $M_{1}+M_{2}$ and the third is $M_{3}+M_{4}$. The resulting $2 \times 2$ matrix is the one actually used in the calculation. It is

$\mathbf{G}_{r}$ (rigid end bonds) $=\left[\begin{array}{cc}\left(\frac{\mu_{1}}{\mu_{1}+\mu_{2}}\right)_{\mu_{2}+\mu_{3}} & -\mu_{3} \\ -\mu_{3} & \mu_{3}+\mu_{4}\left(\frac{\mu_{4}}{\mu_{4}+\mu_{3}}\right)\end{array}\right]$

The stretching force constant matrix to be used with eq (86) is that $\mathbf{F}$, as given by eq (30).

The $\mathbf{G}_{\psi}$ matrix elements for this 5 point case are

$$
\begin{aligned}
& \left(\mathbf{G}_{\psi}\right)_{11}=\varrho_{a}^{2} \mu_{1}+\varrho_{b}^{2} \mu_{3}+\left(\varrho_{a}+\varrho_{b}\right)^{2} \mu_{2} \\
& \left(\mathbf{G}_{\psi}\right)_{22}=\varrho_{b}^{2} \mu_{2}+\varrho_{c}^{2} \mu_{4}+\left(\varrho_{b}+\varrho_{c}\right)^{2} \mu_{3} \\
& \left(\mathbf{G}_{\psi}\right)_{33}=\varrho_{c}^{2} \mu_{3}+\varrho_{d}^{2} \mu_{5}+\left(\varrho_{c}+\varrho_{d}\right)^{2} \mu_{4} \\
& \left(\mathbf{G}_{\psi}\right)_{12}=-\varrho_{b}\left[\left(\varrho_{a}+\varrho_{b}\right) \mu_{2}+\left(\varrho_{b}+\varrho_{c}\right) \mu_{3}\right] \\
& \left(\mathbf{G}_{\psi}\right)_{23}=-\varrho_{c}\left[\left(\varrho_{b}+\varrho_{c}\right) \mu_{3}+\left(\varrho_{c}+\varrho_{d}\right) \mu_{4}\right] ;\left(\mathbf{G}_{\psi}\right)_{13}=\varrho_{b} \varrho_{c} \mu_{3}
\end{aligned}
$$


There are the expressions used in the calculation. Actually, they were not derived from eq (81) but were obtained from Wilson et. al. [12]. However, eq (81) was used for a numerical check of eq (87). To get the matrix elements for the two 4 mass point cases, simply delete from eq (87) those elements which contain either a missing $\varrho$ or a missing $\mu$ or both. Do the same for the 3 point case, but delete also $\left(G_{\psi}\right)_{13}$; (there is only one element, $\left(\mathbf{G}_{\psi}\right)_{22}$, in this case).

We are now ready to consider the secular equation. For the rate constant calculation only the $\lambda_{k}$ are required, so that a solution of eq (54) for the transformation matrix $\mathbf{L}$ is not necessary. Nevertheless, $\mathbf{L}$ is easily obtained and is convenient to have for the purpose of illustrating the actual vibrational motions of the complex. Thus we shall solve eq (54) as well as eq (56). According to eq (84), there are two secular equations to be solved ( $\mathbf{H}_{\psi}$ and $\mathbf{H}_{\psi}$, are equal). Because we are using rigid $a$ and $d$ bonds, the dimension of $\mathbf{H}_{r}$ is $2 \times 2$. The maximum dimension of $\mathbf{H}_{\psi}$ is $3 \times 3$ and occurs for the 5 point model. Thus a solution of a $3 \times 3$ problem will suffice for our purpose and will also illustrate how an $n \times n$ problem is to be solved.

We begin by assuming that eq (56) has been solved. In the present work this was accomplished by expanding (56) and solving the resulting polynomial in $\lambda$. In our case, the maximum degree was cubic, so that this part of the calculation was easily performed. As eq (47) shows, the desired frequencies are $\nu_{k}=\lambda_{k}^{1 / 2} / 2 \pi$. For the stretching modes of the complex one of the two frequencies will be imaginary because its $\lambda_{k}$ value will be negative. As mentioned earlier, this corresponds to the asymmetric stretch.

Consider now eq (55) for a general $3 \times 3 \mathbf{H}$ matrix. Written out in full, it is

$$
\begin{gathered}
\left(H_{11}-\lambda_{k}\right) L_{1 k}+H_{12} L_{2 k}+H_{13} L_{3 k}=0 \\
H_{21} L_{1 k}+\left(H_{22}-\lambda_{k}\right) L_{2 k}+H_{23} L_{3 k}=0 \\
H_{31} L_{1 k}+H_{23} L_{2 k}+\left(H_{33}-\lambda_{k}\right) L_{3 k}=0
\end{gathered}
$$

where $\lambda_{k}$ is one of the three values of $\lambda$ determined from the solution of the cubic (in this case) eq (56). Divide the first two of these equations by $L_{3 k}$, and define the ratios $g_{i k}=L_{i k} / L_{3 k}$. This yields two equations to be solved for the two unknowns $g_{1 k}$ and $g_{2 k}$.

$$
\begin{gathered}
\left(H_{11}-\lambda_{k}\right) g_{1 k}+H_{12} g_{2 k}=-H_{13} \\
H_{21} g_{1 k}+\left(H_{22}-\lambda_{k}\right) g_{2 k}=-H_{23}
\end{gathered}
$$

We get two $g_{i k}$ values for each value of $\lambda_{k}$ substituted into eq (89), or six $g_{i k}$ values in all. Using these values, we can express $L$ in terms of the product of two matrices defined by

$\mathbf{L}=\left[\begin{array}{lll}g_{11} & g_{12} & g_{13} \\ g_{21} & g_{22} & g_{23} \\ 1 & 1 & 1\end{array}\right] \quad\left[\begin{array}{lll}L_{31} & 0 & 0 \\ 0 & L_{32} & 0 \\ 0 & 0 & L_{33}\end{array}\right]=\Gamma l$

To determine the components of $\mathfrak{h}$, insert eq $(90)$ into eq (51). We get

$$
\text { lf } \Gamma+F \Gamma \ell=\Lambda=\ell+\ell \Gamma+F \Gamma
$$

The final reordering is possible because $\ell$ and $\Lambda$ are diagonal and therefore $\Gamma \nmid F \Gamma$ is diagonal. This equation is easily solved for the elements $\ell+\ell$ to give

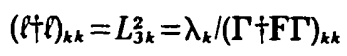

The other elements of $\mathbf{L}$ are gotten from these values and the ratios $\boldsymbol{g}_{i k}$ already determined.

The actual motions in the cartesian system can now be obtained by combining eq (39) with the inverse of eq (60) or eq (77). For the stretching motions we have 


$$
\mathbf{X}=\mathbf{M}^{-1} \tilde{\mathbf{R}}=\mathbf{M}^{-\mathbf{1}}\left[\begin{array}{c}
\mathbf{L}_{r} \mathbf{Q}_{r} \\
0
\end{array}\right]
$$

where $\mathbf{L}_{r}$ arises from the secular equation containing $\mathbf{H}_{r} . \mathbf{Q}_{r}$ is the normal coordinate vector and the $x$-component of the center of mass has been set to zero. A similar equation results for the bending modes. This is

$$
\mathbf{Y}=\mathbf{A}^{-1} \widetilde{\Psi}=\mathbf{A}^{-1}\left[\begin{array}{c}
\mathbf{L}_{\psi} \mathbf{Q}_{\psi} \\
0 \\
0
\end{array}\right]
$$

where the $z$-component of the angular momentum and the $y$-component of the center of mass have been set to zero.

This completes the frequency analysis. In the next section we will consider the partition functions.

\subsection{Partition Functions.}

Herschbach et. al. [13] have shown how to express the classical partition function for polyatomic molecules in terms of local properties. We shall use their method because it allows for cancellations of considerable portions of the partition functions of the complex and reactants when their ratios are evaluated in the rate constant expression, eq (3). We begin the discussion with the classical partition function for a linear polyatomic molecule. This is (see Herzberg [14], pp. 502-509),

$$
q_{c l}=q_{e} V\left(2 \pi M k T / h^{2}\right)^{3 / 2}(k T /(\sigma h c B)) \prod_{i}^{3 N-5}\left(k T /\left(\omega_{i} h c\right)\right)
$$

where $q_{e}$ is the electronic partition function, $V$ is the volume, $M$ is the total mass of the molecule, $c$ is the velocity of light, $\omega_{i}$ is the frequency of the $i$ th vibrational mode in $\mathrm{cm}^{-1}\left(\omega_{i}=\nu_{i} / c\right), N$ is the number of atoms in the molecule, $B$ is the rotational constant; $B=h\left(8 \pi^{2} c I\right)$, where $I$ is the moment of inertia of the molecule; $\sigma$ is the symmetry number which is the number of indistinguishable positions into which the molecule can be turned by simple rigid rotations. For linear molecules $\sigma=1$ or 2 . Equation (95) neglects nuclear spins, anharmonicity, and non-rigidity of the molecule. Let us rewrite eq (95) in terms of $I$ and $u_{i}=h \nu_{i} / k T$. It becomes

$$
q_{c l}=q_{e} V 4 \pi \sigma^{-1}\left(2 \pi k T h^{-2}\right)^{5 / 2} M^{3 / 2} \mathrm{I} \prod_{i}^{3 N-5} u_{i}^{-1}
$$

$q_{c l}$ can also be written in the form

$$
q_{c l}=q_{a} \sigma^{-1} Z \prod_{\alpha}^{N} \Lambda_{a}^{-3}
$$

where

$$
\begin{gathered}
Z=\int \ldots \ldots \int e^{-v / k T} d x_{1} \ldots \ldots d z_{n} \\
\Lambda_{\alpha}=h\left(2 \pi M_{\alpha} k T\right)^{-1 / 2}
\end{gathered}
$$

$Z$ is the so-called configuration integral, $V$ is the potential energy, and $x_{1}, y_{1}, z_{1}, \ldots \ldots x_{N}, y_{N}, z_{N}$ are the cartesian coordinates of each of the $N$ atoms. Eliminating $q_{c l}$ between eqs (96) and (97) gives

$$
Z=V 4 \pi\left(2 \pi k T h^{-2}\right)^{-1 / 2(3 N-5)} M^{3 / 2} I \prod_{a}^{N} M_{a}^{-3 / 2} \prod_{i}^{3 . N-5} u_{i}^{-1}
$$

Consider now the matrix $\mathbf{H}=\mathbf{G F}$ defined by eq (84). A theorem of matrix algebra states that the determinant of $\mathbf{H}$ equals the product of its eigenvalues (see Hohn [15], p. 283). There is also a theorem (Hohn, p. 65), 
stating that the determinant of product of two matrices equals the products of the determinants of the matrices in the product. Consequently,

$$
|\mathbf{H}|^{1 / 2}=|\mathbf{G}|^{1 / 2}|\mathbf{F}|^{1 / 2}=\prod_{i}^{3 N-5} \lambda_{i}^{3 / 2}=(2 \pi k T / h)^{3 N-5} \prod_{i}^{3 N-5} u_{i}
$$

Solving for the product over $u_{i}$ gives

$$
\prod_{i}^{3 N-5} u_{i}^{-1}=(2 \pi k T / h)^{3 N-5}|\mathbf{G}|^{-1 / 2}|\mathbf{F}|^{-1 / 2}
$$

Inserting eq (101) into (99) yields

$$
Z=V 4 \pi(2 \pi k T)^{1 / 2(3 N-5)} M^{3 / 2} I \prod_{\alpha}^{N} M_{\alpha}^{-3 / 2}|\mathbf{G}|^{-1 / 2}|\mathbf{F}|^{-1 / 2}
$$

This can be rearranged to give

$$
|\mathbf{F}|^{1 / 2}(2 \pi k T)^{-1 / 2(3 N-5)} Z=V 4 \pi M^{3 / 2} I \prod_{\alpha}^{N} M_{\alpha}^{-3 / 2}|\mathbf{G}|^{-1 / 2}=\mathbf{J}_{N}
$$

The left side of eq (103) does not involve the masses, while the right side does not contain force constants. Therefore, the quantity denoted by $J_{N}$ does not depend on either the force constants or the masses, but must depend only on geometrical parameters. Herschbach et. al. [13] have shown that for linear molecules

$$
J_{N}=V 4 \pi \prod_{i=1}^{N-1} R_{i+1, i}^{2}
$$

where $R_{i+1, i}$ is the equilibrium distance between mass $M_{i}$ and $M_{i+1}$. For a general linear molecule, the classical partition function per unit volume can now be written

$$
Q_{c l}=q_{c l} / V=V^{-1} q_{e} \sigma^{-1} J_{N}(2 \pi k T)^{1 / 2(3 N-5)}|\mathbf{F}|^{-1 / 2} \prod_{\alpha}^{N} \Lambda_{\alpha}^{-3}
$$

This form of the partition function is suitable for the reactant molecules.

Let us now consider the partition function for the complex. Using eq (96), we have

$$
k T h^{-1} q^{*}=k T h^{-1} q_{\cdot} \sigma^{-1} V 4 \pi\left(2 \pi k T h^{-2}\right)^{5 / 2} M^{3 / 2} I \stackrel{3 N-6}{\prod_{i}^{-6}} u_{i}^{-1}
$$

Note that the product is over $3 N-6$; i.e., one less vibration than in a stable linear molecule. Consider next the quantity

$$
\begin{aligned}
\left(\prod_{i}^{3 N-6} u_{i}^{-1}\right) k T h^{-1} & =(k T / h)^{3 N-5} \prod_{i}^{3 N-6} \nu_{i}^{-1}=(k T / h)^{3 N-5} \prod_{i}^{3 N-6} 2 \pi \lambda_{i}^{-1 / 2} \\
& =(k T / h)^{3 N-5}(2 \pi)^{3 N-6} \lambda^{* 1 / 2} \prod_{i}^{3 N-6} \lambda_{i}^{-1 / 2} \lambda^{*-1 / 2} \\
& =(2 \pi k T / h)^{3 N-5} \nu^{*}|\mathbf{F}|^{-1 / 2}|\mathbf{G}|^{-1 / 2}
\end{aligned}
$$

where $\lambda^{*}$ is the negative eigenvalue and $\nu^{*}$ is the associated imaginary frequency; eq $(100)$ has been used. Using eq (107) in (106) gives

$$
\begin{gathered}
k T h^{-1} Q^{*}=k T h^{-1} q^{*} V^{-1}=V^{-1} q . \sigma^{-1}\left[V 4 \pi M^{3 / 2} I \prod_{\alpha}^{N} M_{\alpha}^{-3 / 2}|\mathbf{G}|^{-1 / 2}\right] \nu^{*}|\mathbf{F}|^{-1 / 2}(2 \pi k T)^{3 N-5 / 2} \\
\cdot h^{-3 N} \prod_{\alpha}^{N} M_{\alpha}^{3 / 2} \\
=V^{-1} q_{e} \sigma^{-1} J_{N} \nu^{*}|\mathbf{F}|^{-1 / 2}(2 \pi k T)^{1 / 2(3 N-5)} \prod_{\alpha}^{N} \Lambda_{\alpha}^{-3}
\end{gathered}
$$


This equation is very similar to eq (105), the partition function of a stable molecule. Note that $|\mathbf{F}|^{-1 / 2}$ will be imaginary for the complex.

We can now write down the specific partition functions per unit volume for the four reaction cases shown in figure $1 \mathrm{~b}$.

\section{Case III.}

Species $A=M_{2}-M_{3}$

Species $B=M_{4}$

Species $C=M_{2} \ldots M_{3} \ldots M_{4}$

$Q_{A}=q_{a A} \sigma_{A}^{-1} 4 \pi R_{b s}^{2}(2 \pi k T)^{1 / 2} F_{b s}^{-1 / 2}\left(\Lambda_{2} \Lambda_{3}\right)^{-3}$

$Q_{B}=q_{e B} \Lambda_{4}^{-3}$

$Q_{c} k T / h=q_{c c} \sigma_{c}^{-1} 4 \pi R_{b}^{2} R_{c}^{2} \nu^{*}\left|\mathbf{F}_{r}\right|^{-1 / 2} F_{\psi_{3}}^{-1}(2 \pi k T)^{2}\left(\Lambda_{2} \Lambda_{3} \Lambda_{4}\right)^{-3}$

The matrix $F_{r}$ is the $2 \times 2$ one given by eq. (5b), and not the $4 \times 4$ used in eq (83).

Case IVa.

Species $A=M_{1}-M_{2}-M_{3}$

Species $B=M_{4}$

Species $C=M_{1}-M_{2} \ldots M_{3} \ldots M_{4}$

$Q_{A}=q_{e A} \sigma_{A}^{-1} 4 \pi R_{a s}^{2} R_{b s}^{2}(2 \pi k T)^{2}\left(F_{a s} F_{b s}\right)^{-1 / 2} F_{\psi_{2 s}}^{-1}\left(\Lambda_{1} \Lambda_{2} \Lambda_{3}\right)^{-3}$

$Q_{B}=q_{\text {eB }} \Lambda_{4}^{-3}$

$Q_{c} k T / h=q_{c c} \sigma_{c}^{-1} 4 \pi R_{a s}^{2} R_{b}^{2} R_{c}^{2} \nu^{*} F_{a s}^{-1 / 2}\left|\mathbf{F}_{r}\right|^{-1 / 2} F_{\psi_{2}}^{-1} F_{\psi_{3}}^{-1}(2 \pi k T)^{7 / 2}\left(\Lambda_{1} \Lambda_{2} \Lambda_{3} \Lambda_{4}\right)^{-3}$

Note that $I$ have included $F_{a s}$ in $Q_{A}$ and $Q_{C}$ even though it is supposed to be infinite; it will cancel out when the ratio $Q_{C} / Q_{A}$ is taken. Also note that the bending force constants appear with twice the power of the stretching force constants. This is because of the degeneracy.

Case IVb.

Species $A=M_{2}-M_{3}$

Species $B=M_{4}-M_{5}$

Species $C=M_{2} \ldots M_{3} \ldots M_{4}-M_{5}$

$Q_{A}=q_{e A} \sigma_{A}^{-1} 4 \pi R_{b s}^{2}(2 \pi k T)^{1 / 2} F_{b s}^{-1 / 2}\left(\Lambda_{2} \Lambda_{3}\right)^{-3}$

$Q_{B}=q_{e B} \sigma_{B}^{-1} 4 \pi R_{d s}^{2}(2 \pi k T)^{1 / 2} F_{d s^{-1 / 2}}^{-1 /}\left(\Lambda_{4} \Lambda_{5}\right)^{-3}$

$Q_{c} k T / h=q_{e c} \sigma_{c}^{-1} 4 \pi R_{b}^{2} R_{c}^{2} R_{d s}^{2} \nu^{*}\left|F_{r}\right|^{-1 / 2} F_{\psi_{3}}^{-1} F_{\psi_{4}}^{-1}(2 \pi k T)^{7 / 2}\left(\Lambda_{2} \Lambda_{3} \Lambda_{4} \Lambda_{s}\right)^{-3} F_{d s}^{-1 / 2}$

\section{Case V.}

Species $A=M_{1}-M_{2}-M_{3}$

Species $B=M_{4}-M_{5}$

Species $C=M_{1}-M_{2} \ldots M_{3} \ldots M_{4}-M_{5}$

$Q_{A}=q_{e A} \sigma_{A}^{-1} 4 \pi R_{a s}^{2} R_{b s}^{2}(2 \pi k T)^{2}\left(F_{a s} F_{b s}\right)^{-1 / 2} F_{v / 2}^{-1}\left(\Lambda_{1} \Lambda_{2} \Lambda_{3}\right)^{-3}$

$Q_{B}=q_{e B} \sigma_{B}^{-1} 4 \pi R_{d s}^{2}(2 \pi k T)^{1 / 2} F_{d k^{-1 / 2}}\left(\Lambda_{4} \Lambda_{s}\right)^{-3}$

$Q_{c} k T / h=q_{c c} \sigma_{C}^{-1} 4 \pi R_{a s}^{2} R_{b}^{2} R_{c}^{2} R_{d s}^{2} \nu^{*} F_{a s}^{-1 / 2}\left|\mathbf{F}_{r}\right|^{-1 / 2} F_{d d^{-1 / 2}} F_{\psi_{2}}^{-1} F_{\psi_{3}}^{-1} F_{\psi_{4}}^{-1}(2 \pi k T)^{s}\left(\Lambda_{1} \Lambda_{2} \Lambda_{3} \Lambda_{4} \Lambda_{s}\right)^{-3}$

We now have everything for eq (3) except the tunneling correction. This will be taken up in the next section.

\subsection{Tunneling Correction}

The one-dimensional Eckart potential function was used to approximate the barrier to quantum mechanical tunneling from reactants to products. Three parameters are required for its definition; these are shown in figure 8 . Its functional form is 


$$
V(x)=\frac{A y}{1-y}-\frac{B y}{(1-y)^{2}}
$$

where

$$
\begin{aligned}
& y=-e^{2 \pi x / L} \\
& A=V_{1}-V_{2} \\
& B=\left(V_{1}^{1 / 2}+V_{2}^{1 / 2}\right)^{2} \\
& L=2 \pi(-2 / F)^{1 / 2}\left(V_{1}^{-1 / 2}+V_{2}^{-1 / 2}\right)^{-1}, \text { and } \\
& F=\frac{\partial^{2} V}{\partial x^{2}}
\end{aligned}
$$

evaluated at the maximum in the curve. $F$ is a force constant. Using this potential function, Eckart [16] solved the wave equation and obtained the transmission coefficient for a particle with mass $m$ approaching the barrier from the left with an energy $E$. His result is

$$
\mathbf{K}\left(E, V_{1}, V_{2}, F\right)=1-\frac{\cosh \left[2 \pi\left(\alpha_{1}-\alpha_{2}\right)\right]+\Delta}{\cosh \left[2 \pi\left(\alpha_{1}+\alpha_{2}\right)\right]+\Delta}
$$

where $\Delta=\cosh [2 \pi \delta]$ if $\delta$ is real, and $\Delta=\cos [2 \pi|\delta|]$ if $\delta$ is imaginary. The relationships of $\alpha_{1}, \alpha_{2}$, and $\delta$ to the parameters of figure 8 , are

$$
\begin{aligned}
\alpha_{1} & =1 / 2(E / C)^{1 / 2} \\
\alpha_{2} & =1 / 2[(E-A) / C]^{1 / 2} \\
\delta & =1 / 2[(B-C) / C]^{1 / 2} \\
C & =h^{2} /\left(8 m L^{2}\right)
\end{aligned}
$$

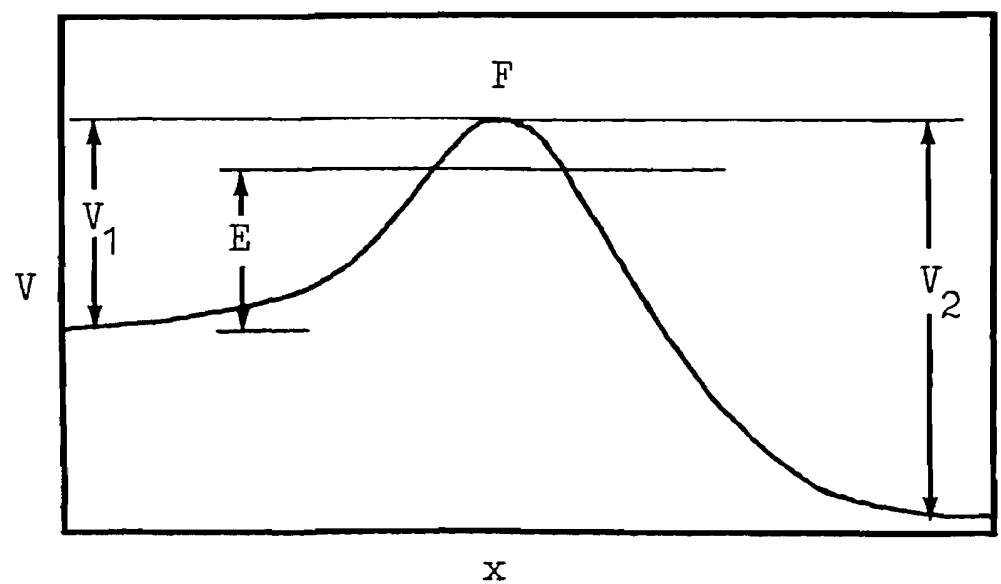

Figure 8. Eckart potential function.

Given the transmission coefficient, Johnston [2], pp. 42 and 43 , has derived the correction factor $\Gamma^{*}$ which is the ratio of the quantum barrier crossing rate to the classical crossing rate. His result is

$$
\Gamma^{*}=e^{V_{1} / k T} \int_{\varepsilon_{o}}^{\infty} \mathbf{K}(E) e^{-E / k T} d E / k T
$$

$$
\text { where } E_{o}=0 \text { when } V_{1} \leq V_{2} \text { and } E_{0}=V_{1}-V_{2} \text { when } V_{1}>V_{2}
$$


Let us rewrite this in a more symmetrical form. We define a new variable $\epsilon=\left(E-V_{1}\right) / k T, E=k T \epsilon+V_{1}$. Equation (112) becomes

$$
\Gamma^{*}=\int_{\varepsilon_{0}}^{\infty} \mathbf{K}(\epsilon) e^{-\epsilon} d \epsilon
$$

where $\epsilon_{o}=-V_{1} \mid k T$ when $V_{1} \leq V_{2}$ and $\epsilon_{o}=-V_{2} \mid k T$ when $V_{1}>V_{2}$.

With this substitution, the parameters $\alpha_{1}$ and $\alpha_{2}$ become

$$
\alpha_{i}=1 / 2\left(k T \epsilon / C+V_{i} / C\right)^{1 / 2}, \quad i=1 \& 2
$$

From eq (110) we have

$$
\mathbf{K}=\mathbf{K}\left(\alpha_{1}, \alpha_{2}, \delta\right)=\mathbf{K}\left(k T_{\epsilon} / C, V_{1} / C, V_{2} / C, B / C\right)
$$

But

$$
B / C=V_{1} / C+2\left[\left(V_{1} / C\right)\left(V_{2} / C\right)\right]^{1 / 2}+V_{2} / C
$$

is a function of $V_{1} / C$ and $V_{2} / C$. Therefore

$$
\mathbf{K}=\mathbf{K}\left(\epsilon, p, p_{1}, p_{2}\right)
$$

where

$$
p=k T / C, p_{1}=V_{1} / C \text { and } p_{2}=V_{2} / C .
$$

$\Gamma^{*}$ thus depends on three parameters. Furthermore, it is invariant when $p_{1}$ and $p_{2}$ are interchanged; i.e., $\Gamma^{*}\left(p, p_{1}, p_{2}\right)=\Gamma^{*}\left(p, p_{2}, p_{1}\right)$. To see this let $p_{1}^{\prime}=p_{2}$ and $p_{2}^{\prime}=p_{1}$. From eq (115) we see that

$$
(B / C)^{\prime}=p_{1}^{\prime}+2\left(p_{1}^{\prime} p_{2}^{\prime}\right)^{1 / 2}+p_{2}^{\prime}=p_{2}+2\left(p_{2} p_{1}\right)^{1 / 2}+p_{1}=B / C
$$

Thus, $\delta^{\prime}=\delta$. From eq (114) we have

$$
\begin{aligned}
& \alpha_{1}^{\prime}=1 / 2\left(p \epsilon+p_{1}^{\prime}\right)^{1 / 2}=1 / 2\left(p \epsilon+p_{2}\right)^{1 / 2}=\alpha_{2} \\
& \alpha_{2}^{\prime}=\alpha_{1}
\end{aligned}
$$

Using these results in eq (110), we get

$$
\mathbf{K}\left(\epsilon, p, p_{1}^{\prime}, p_{2}^{\prime}\right)=\mathbf{K}\left(\epsilon, p, p_{2}, p_{1}\right)=\mathbf{K}\left(\epsilon, p, p_{1}, p_{2}\right)
$$

Suppose that $p_{1}^{\prime}>p_{2}^{\prime}$; i.e., $V_{1}^{\prime}>V_{2}^{\prime}$. Using eq (117), eq (113) becomes

$$
\Gamma^{*}\left(p, p_{1}^{\prime}, p_{2}^{\prime}\right)=\int_{-p_{1}^{\prime} / p}^{\infty} \mathbf{K}\left(\epsilon, p, p_{1}^{\prime}, p_{2}^{\prime}\right) e^{-\epsilon} d \epsilon=\int_{-p_{1} / p}^{\infty} \mathbf{K}\left(\epsilon, p, p_{1}, p_{2}\right) e^{-\epsilon} d \epsilon=\Gamma^{*}\left(p, p_{1}, p_{2}\right)
$$

The way Eq (113) was integrated to get $\Gamma^{*}$ will be considered later when the computer program is discussed.

In applying this correction, it is assumed that the $x$ coordinate of Eckart's potential lies in the $\varrho$ direction discussed earlier. This is that direction at the saddle point in which the potential energy decreases most rapidly. It is also the direction of the path of constant total bond order. We therefore use the force constant $F_{\text {o }}$ given by eq $(22)$ for the second derivative of the Eckart potential at its maximum. The effective mass for tunneling, $M_{r}$, is the proportionality factor between the kinetic energy and $1 / 2 \dot{Q}^{2}$. We can calculate $M_{r}$ in the following way: As far as tunneling is concerned, in the 4 and 5 mass point cases there are effectively 3 
masses, since the end bonds are supposed to be rigid. Thus, there are only the two variables, $R_{b}$ and $R_{c}$, involved. (Bending modes are not considered.) The kinetic energy $T$, for changes in these two bonds is given by

$$
T_{r}=1 / 2 \dot{\mathbf{R}}+\mathbf{G}_{r}^{-} \dot{\mathbf{R}}
$$

where $\mathbf{R}$ is the 2 -dimensional vector defined by eq (6), and $\mathbf{G}_{r}$ is the $2 \times 2$ matrix given by eq (86). The inverse of this matrix is easily calculated and found to be

$$
\mathbf{G}_{r}^{-1}=\left[\begin{array}{cc}
M_{2}^{\prime}\left(M_{3}+M_{4}^{\prime}\right) & M_{2}^{\prime} M_{4}^{\prime} \\
M_{2}^{\prime} M_{4}^{\prime} & \left(M_{2}^{\prime}+M_{3}\right) M_{4}^{\prime}
\end{array}\right] \mathrm{M}^{-1}
$$

where $M_{2}^{\prime}=M_{1}+M_{2}$ and $M_{4}^{\prime}=M_{4}+M_{5}$ in the 5 point case. The transformation between $R_{b}, R_{c}$ and $\varrho, \sigma$ is given by the matrix $U$ whose value, determined by the BEBO calculation, is given by eq (18). $U$ can be used to express $T$, in terms of $\varrho$ and $\sigma$. Thus

$$
T_{r}=1 / 2 \dot{\mathbf{R}}+\mathbf{G}_{r}^{-1} \dot{\mathbf{R}}=1 / 2 \dot{\mathbf{P}}_{\mathbf{U}}+\mathbf{G}_{r}^{-1} \mathbf{U} \dot{\mathbf{P}}
$$

The desired quantity $M_{\mathrm{r}}$ is simply the matrix element $\left(\mathbf{U}+\mathbf{G}_{r}^{-1} \mathbf{U}\right)_{\dot{Q} \dot{e}^{*}}$ This is

$$
M_{s}=\frac{M_{2}^{\prime}\left(M_{3}+M_{4}^{\prime}\right) m^{2}-2 M_{2}^{\prime} M_{4}^{\prime} n m+\left(M_{2}^{\prime}+M_{3}\right) M_{4}^{\prime} n^{2}}{\left(n^{2}+m^{2}\right) M}
$$

where $n$ and $m$ are the bond orders from the BEBO calculation, and $M$ is the total mass of the molecule.

The bases from which the tunneling parameters $V_{1}$ and $V_{2}$ are measured are taken to be the zero point energies of the reactants and products, respectively, and not the potential minimums as might be expected. The maximum of the potential, on the other hand, is placed at the potential minimum of the complex; i.e., at the saddle point. Johnston [2], pp. 190-196, gives reasons for this particular method of using the Eckart function for tunneling corrections.

We finally have everything needed for eq (3). In the next section explicit rate constant expressions will be given for the four reaction cases of figure 2 .

\subsection{Rate Constant Expressions}

The rate constant expression eq (3) is not quite complete. It should be multiplied by the number of equivalent $\mathrm{H}$ atoms on the molecule being attacked. Let us call this factor the chemical multiplicity, $\sigma_{c h}$. For example, there are 6 identical reaction paths for $\mathrm{H}$ abstraction of the 6 terminal $\mathrm{H}$ atoms on propane, and 2 paths for abstraction of the 2 central $\mathrm{H}$ atoms. Thus $\sigma_{c h}=6$ in the first case, and 2 in the second. With this factor added, the rate constants for the four cases shown in figure 2 are

Case III. $M_{2}-M_{3}+M_{4}-M_{2} \ldots M_{3} \ldots M_{4}$

$$
\mathbf{k}=\mathrm{S} F_{\psi_{3}}^{1}(2 \pi k T)^{3 / 2}\left(\Gamma_{3}^{3}\right)^{2}
$$

Case IVa. $M_{1}-M_{2}-M_{3}+M_{4}-M_{1}-M_{2} \ldots M_{3} \ldots M_{4}$

$$
\mathbf{k}=\mathrm{S} F_{\psi_{2}}\left(F_{\psi_{2}} F_{\psi_{3}}\right)^{-1}(2 \pi k T)^{3 / 2}\left(\Gamma_{2} \Gamma_{3}^{3}\right)^{2}\left(\Gamma_{2}^{A}\right)^{-2}
$$

Case IVb. $M_{2}-M_{3}+M_{4}-M_{5} \rightarrow M_{2} \ldots M_{3} \ldots M_{4}-M_{5}$

$$
\mathbf{k}=S\left(F_{\downarrow_{3}} F_{\psi_{4}}\right)^{-1}(2 \pi k T)^{5 / 2}\left(\Gamma_{3} T_{4}\right)^{2}(4 \pi)^{-1}
$$


Case V. $M_{1}-M_{2}-M_{3}+M_{4}-M_{5} \rightarrow M_{1}-M_{2} \ldots M_{3} \ldots M_{4}-M_{5}$

$$
\mathbf{k}=S F_{\downarrow_{2}}\left(F_{\downarrow_{2}} F_{\downarrow_{3}} F_{\downarrow_{4}}\right)^{-1}(2 \pi k T)^{5 / 2}\left(\Gamma_{2}^{*} \Gamma_{3}^{*} \Gamma_{4}^{*}\left(\Gamma_{2}^{A}\right)^{-2}(4 \pi)^{-1}\right.
$$

The common factor in all these expressions is

$$
\mathrm{S}=\sigma_{c h} \frac{q_{e c} \sigma_{A} \sigma_{B} R_{b}^{2} R_{c}^{2}}{q_{e A} q_{e B} \sigma_{C} R_{b s}^{2}} \nu^{*} \frac{F_{b s}^{1 / 2} \Gamma_{s}^{*}}{\left|\mathbf{F}_{r}\right|^{1 / 2} \Gamma_{s}^{A}} \Gamma^{*} e^{-v^{*} / k T}
$$

The calculated factors in $S$ are:

1) $R_{b}$ and $R_{c}$; these are calculated from $n$ and $m$ through Pauling's relation, eq (9).

2) $\nu^{*}$ is the imaginary frequency obtained from the vibration analysis for the asymmetric stretch.

3) $\left|\mathbf{F}_{r}\right|$ is the determinant of the matrix given by eq (30). It is negative.

4) $\Gamma_{s}^{*}$ is the quantum correction factor for the symmetric stretching frequency obtained from the vibrational analysis.

5) $\Gamma^{*}$ is the tunneling correction factor obtained in section 2.5 .

6) $V^{*}$ is the saddle point potential energy given by the BEBO calculation.

Other calculated factors are:

1) $F_{\psi_{3}}$ is the bending force constant given by eq (33).

2) $F_{\psi_{2}}$ and $F_{\psi_{4}}$ are the bending force constants given by eq (34).

3) The quantum correction factors $\Gamma_{2}^{*}, \Gamma_{3}^{*}, \Gamma_{4}^{*}$ for the bending modes come from the frequency analysis via eq (2).

This concludes the theoretical part of this discussion. The next section contains a brief discussion of the computer program which was written to implement the rate constant calculations. This will be followed by instructions on how to use it.

\section{Computer Implementation of BEBO}

The computer program consists of a main section and six subroutines. It is written in an enhanced form of BASIC for use on a Hewlett-Packard $9845 \mathrm{~A}$ computer.

\subsection{Description of the Main Program}

The main program begins by reading the following data:

1) Runid\$

This is a string variable having up to 79 alphanumeric characters to be used for the run identification.

2) $0 p t(M), M=1,7$

These are flags which provide a series of available options. These will be described in detail in the instruction section.

3) Ntemp

This is the number of temperature values at which the rate constant is to be evaluated. A maximum of 16 values will be allowed.

4) $T$ min, $T$ max

The minimum and maximum temperature values desired. The reciprocal temperature scale is divided into Ntemp - 1 equal intervals and the temperature evaluated from the reciprocal values. This gives a better distribution on an Arrhenius plot than if the temperature scale were divided into equal intervals.

5) M1,M2,M3,M4,M5

These are the five mass point values determined according to the rules given in section 2.1.

6) Ras, Rbs, Rcs, Rds

These are the equilibrium bond distances for single bonds.

7) Ebs, Ecs,P,Q 
The first two parameters are the electronic energies for single bonds $b$ and $c$; the last two are the BEBO parameters obtained from eq (12).

8) Rts, Ets, Beta

These are the bond distance, bond energy, and Morse parameter $\beta$ for the triplet interaction.

9) Fbs,Fcs,Fpsi2s,Fpsi4s

These are the stretching force constants for single $b$ and $c$ bonds, and the bending force constants about the M2 and M4 masses.

10) $\mathrm{Sa}, \mathrm{Sb}, \mathrm{Sc}$

These are the partition function symmetries $\sigma_{A}, \sigma_{B}$, and $\sigma_{O}$.

11) Schem

This is the chemical multiplicity $o_{c h}$.

12) Sea,Seb,Sec

These are the electronic degeneracies $\mathrm{q}_{e A}, \mathrm{q}_{e B}$, and $\mathrm{q}_{e C}$.

The program next prints out this input data to provide an easily read record and a check of the numbers. After these preliminaries, the program then determines the saddle point position. This is done by an iterative procedure; $n$ is initially set to 0.5 ; then the potential energy $V$ is calculated according to eq (14) along with its first and second derivatives, $V n$ and $V n n$, with respect to $n$. The subroutine Trpl is used to calculate the triplet part of $V$. A new $n$ is estimated by the Newton, Raphson method from the formula $n^{\prime}=n$ $-V_{n} / V n n$. The process is repeated using $n^{\prime}$ and continued until covergence is obtained. This yields a value of $n$ which makes $V n$ zero; this will correspond to the desired maximum in $V$. (I have not investigated the conditions for which a maximum is expected or if there could be more than one maximum.)

Having obtained the value of $n$ for the saddle point, the program calculates the stretching force constant matrix Fr given by eq (30), its determinant, and the saddle values of $R b$ and $R c$ from Pauling's relation eq (9). It then evaluates the mass to be used for tunneling from a somewhat rearranged eq (120). Next, the $2 \times$ 2 matrix $G r$ is calculated from eq (86). This is then combined with $F r$ to form $H r$, and the stretching frequencies obtained by solving the resulting quadratic secular equation. The bending frequencies are next determined through the matrices $F(\mathrm{eq}(35))$ and $\mathrm{G}(\mathrm{eq}(87))$. The sizes of these matrices will depend on the type of reaction. For the three mass point model there is only one element and thus a linear secular equation with one bending frequency. The two four point models require solving a quadratic secular equation for two frequencies. The five point model uses the subroutine Cubic to solve the cubic secular equation for three frequencies. The subroutine Normod then calculates the matrix for the normal coordinate transformation of the stretching modes.

At this point, the program prints out a number of properties of the complex. This will be discussed in detail in the instruction section.

The rate constants are then evaluated from eqs (12l) at the different temperatures. The activation energy is gotten by numerically differentiating the logarithm of the rate constant by means of suitable finite difference formulas. Subroutine Fit is a least-squares routine which is used to fit Arrhenius equations through the calculated points. The program concludes with subroutine Pltk which draws an Arrhenius plot of the results.

\subsection{Discussion of Subroutine Tun}

The only subroutine worth discussing is Tunl, the routine for evaluating the integral of eq (112) for the tunneling correction factor $\Gamma^{*}$. Johnston and Heicklen [17] calculated this integral numerically by an unspecified method for a range of input parameter values. The three input parameters which they used were $h v^{*} / k T$, where $v^{*}=(-F / m)^{1 / 5}(2 \pi), 2 \pi V_{1} l\left(h v^{*}\right)$, and $2 \pi V_{2} J\left(h v^{*}\right)$. Their results are in the form of a table. The method used in the present program is a modified 6-point Gaussain quadrature formula based on Legendre polynomials (see Abramowitz and Stegun [18). This was used even though the nature of the integral sug. gests using a formula based on Laguerre polynomials. Neither of these formulas was satisfactory for the whole range of parameter values given by Johnston and Heicklen, so a modification of the first method was developed. It was based on the following ideas: When $\epsilon$ gets large, the transmission approaches unity. The 
idea is to use the Gaussian formula for that part of the integral where $\mathbf{K}(\epsilon)<1$. After $\mathbf{K}(\epsilon)$ has gotten sufficiently close to unity, the remainder of the integral can be evaluated analytically; i.e., if $\mathbf{K}(\epsilon) \approx 1$ for $\epsilon>\epsilon_{b}$, then

$$
\int_{\epsilon_{b}}^{\infty} \mathbf{K}(\epsilon) e^{-\epsilon} d \epsilon \approx \int_{\epsilon_{b}}^{\infty} e^{-\epsilon} d \epsilon=e^{-\epsilon_{b}}
$$

The problem is to estimate $\epsilon_{b}$. Let us examine eqs (114) as $\epsilon \rightarrow \infty$. We get $\alpha_{i} \rightarrow 1 / 2 \xi^{1 / 2}$, where $\xi=k T_{\epsilon} / C$. From eq (110), we have

$$
\mathbf{K} \rightarrow 1-(1+\Delta)\left(1 / 2 \exp \left(2 \pi \xi^{1 / 2}\right)+\Delta\right)^{-1}=\mathbf{K}_{B}
$$

We can set $\mathbf{K}_{b}$ to some arbitrary value close to unity and solve this equation for $\varepsilon$ and then $\xi$ and then $\varepsilon_{b}$ which will be our cutoff point. The result is

$$
\epsilon_{b}=\mathrm{C}\left\{(2 \pi)^{-1} \ln \left[2(1+\Delta) /\left(1-\mathbf{K}_{b}\right)\right]\right\}^{2}(k \mathrm{~T})^{-1}
$$

It turns out that this value is not entirely satisfactory and subtracting from this the average value of $V_{1}$ and $V_{2}$ works better. Also it can happen that $\epsilon_{b}$ as calculated from this formula can be very large when $K$ is close to unity. Thus, $\exp \left(-\epsilon_{b}\right)$ will be very small. There is no point in using a value for $\epsilon$ as the upper bound to the Gaussian formula if the integrand at this point is negligible because of the exponential factor. Thus $\epsilon_{b}$ was kept below a certain fixed value $\epsilon_{\max }$. This yielded two parameters, $\mathbf{K}_{b}$ and $\epsilon_{\max }$ which were adjusted to minimize the squares of the differences between the results of this method and the results of Johnston and Heicklen. The differences averaged 1.3 percent with only two value differing by as much as 6 percent. Such accurancy should be quite adequate for the rate constant calculations.

\section{INSTRUCTIONS FOR USING BEBO}

\subsection{Input}

It will be assumed that the reader is familiar with the general operation and command system of the HP9845A. The program lines 5000 to 5240 contain a series of DATA statements which hold the input data. As an example, data for the ethane plus methyl radical reaction is contained in these statements. The general nature of the input has been discussed briefly in the last section; here this is considered in more detail.

1) Runid $\$$ is a string variable containing identifying information; 79 characters can be used.

2) $0 p t(M), M=1,7$ are flags for the following options:

Opt(0): This picks out the version of the triplet function $V_{r}$; these different forms of $V_{t}$ will be discussed in the Appendix.

$O p(1):$ As mentioned earlier, the activation energy Eact at any temperature is obtained by numerically differentiating the logarithm of the rate constants. This is done in either of two ways. The more accurate method evaluates the rate constant three times at each temperature; at the particular point and slightly above and below the point. The derivative is then estimated from a 3 point finite difference formula. This is automatically the method used when only a single temperature point is requested. The second, less accurate, but faster method uses the rate constants calculated at Ntemp (see last section or below) points and uses a 5 point difference formula for the derivative. The more points requested and the narrower the temperature range, the more accurate is this method. The value of $0 \mathrm{pt}(1)$ determines which of these methods will be used. Thus, when

$\operatorname{Opt}(1)=1,5$ point difference formula used to get Eact (fastest method).

$O p t(1)=2,3$ point difference formula used to get Eact (most accurate method). 
$0 p t(2):$ When

$0 \mathrm{pt}(2)=1$, the lnatural logarithm of the rate constant is calculated.

$0 \mathrm{pt}(2)=2$, the logarithm, base 10 of the rate constant is calculated.

Opt(3): When

$0 p t(3)=1$, the cathode ray tube is used for the printout. In this mode, execution of the program pauses before the Arrhenius plot is produced, and before the caption to the plot is generated. In each case execution can be resumed by pressing the "cont" key.

$0 \mathrm{pt}(3)=0$, the internal printer is used for the output.

$0 \mathrm{pt}(4)$ : When

$0 \mathrm{pt}(4)=1$, the rate constant is in $\mathrm{cm}^{3} /$ mole-s.

$0 p(4)=2$, the rate constant is in $\mathrm{cm}^{3} /$ molecule-s.

$0 \mathrm{pt}(4)=3$, the rate constant is in liters/mole-s.

$0 p t(4)=4$, the rate constant is in liters/molecule-s.

$0 p t(5):$ Not used.

Opt(6): When

$0 \operatorname{pt}(6)=0$, the Eckart tunneling correction is not applied. It will automatically not be applied if the zero point energy of the reactants is greater than the potential energy $V^{*}$ of the saddle point.

$0 p t(6)=1$, the tunneling correction is applied.

$0 \mathrm{pt}(7):$ When

$\operatorname{Opt}(7)=3$, the three parameter Arrhenius type equation, $A T_{\mathrm{c}-\mathrm{Exact}_{\mathrm{R} T} \mathrm{is}}$ fit to the calculated rate constant values.

$O p t(7)=2$, the standard two parameter Arrhenius equation $A e^{-E a c t \rightarrow R T}$ is fit to the calculated rate constant values.

3) Ntemp is the number of temperature values (up to 16) at which the rate constant is to be evaluated. Use the absolute temperature scale.

4) Tmin, $T \max$ are the minimum and maximum temperature values to be used. If $N$ temp $=1$, then only one temperature value should be entered on this line.

5) $M 1, M 2, M 3, M 4, M 5$ are the five mass point values determined by the rules on page 5 . For 3 point models set $M I$ and $M 5$ to zero. The 4 point models will have either $M 1$ or $M 5$ equal to zero. Atomic mass units are to be used.

6) Ras, $R b s, R c s, R d s$ are the single bond distances in $\dot{A}$. For 3 point models set $R a s$ and $R d s$ to zero. For 4 point models, set either $R a s$ or $R d s$ to zero.

7) $E b s, E c s, P, Q$; the first two parameters are the electronic energies for single bonds in $\mathrm{kcal} / \mathrm{mole}$. The quantity normally available is the bond dissociation energy $D H$ which is defined as the enthalpy change in the process in which one mole of the bond of interest is broken, with reactants and products being in their standard states as ideal gases at $1 \mathrm{~atm}$ and $25^{\circ} \mathrm{C}$. This is not the energy we want. The desired energy $E$ is shown in figure 9, which illustrates the energy relationships involved in the removal of an $\mathrm{H}$ atom from some group $A . Z_{A-H}$ and $Z_{A}$. are the zero point energies for the reactant and molecular product, and $\mathrm{H}_{A-H}^{T}, \mathrm{H}_{A}^{T}$, and $\mathrm{H}_{H}^{T}$. are enthalpies of the speices $A \cdot H, \mathrm{~A} \cdot$, and $\mathrm{H} \cdot$, respectively. In general, a particular enthalpy is the sum of the translational, rotational, vibrational, and $P V$ contributions. We have

$$
H^{T}=H^{T} \text { (trans) }+H^{T}(\text { rot })+H^{T}(\text { vib })+P V
$$

By examing figure 9 it is easy to derive the relationship between $E$ and $D H$. It is

$$
E=D H+\left(H_{A-H}^{T}-H_{A}^{T}\right)+\left(Z_{A-H}-Z_{A} .\right)-H_{H}^{T} .
$$




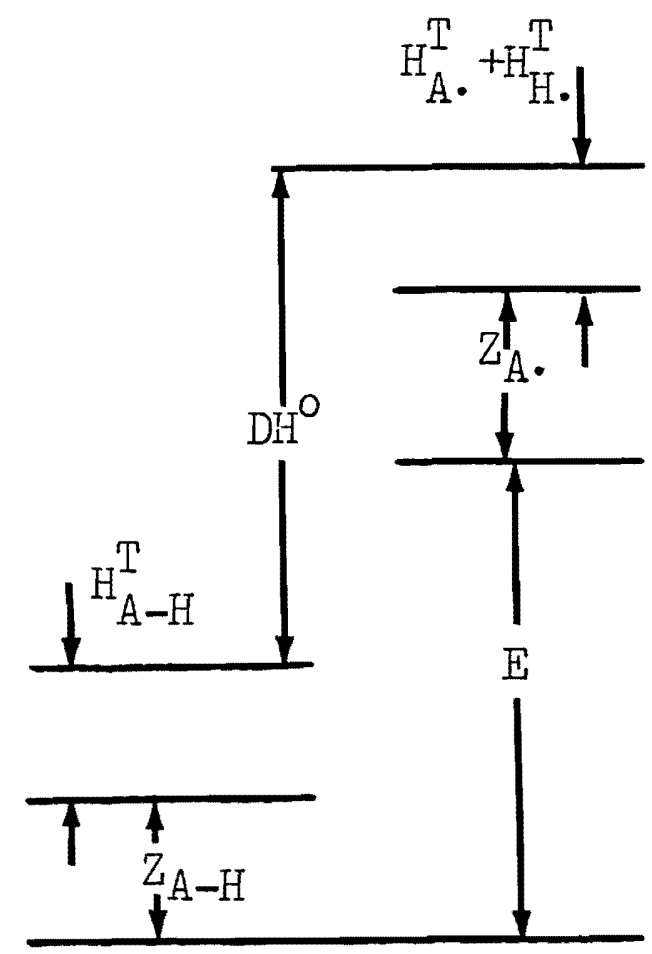

FIcURE 9. Bond energy relationships.

The second term is

$$
\begin{gathered}
\left.H_{A-H}^{T}-H_{A .}^{T}=H_{A-H}^{T}(\text { trans })-H_{A}^{T} \text {. (trans }\right)+H_{A-H}^{T}(\text { rot })-H_{A}^{T} \text { (rot) }+H_{A-H}^{T}(\text { vib }) \\
\left.H_{A-H}^{T}-H_{A}^{T} .=H_{A-H}^{T} \text { (trans }\right)-H_{A}^{T} .
\end{gathered}
$$

Assuming equipartition of energy, the translational and rotational enthalpies will be the same and the difference in vibrational enthalpies will normally be negligible. Thus, the second term in eq (122) can be neglected. The last term $H_{H}^{T} .=E_{H}^{T} .+P V=3 R T / 2+R T$, where $3 R T / 2$ is the translational energy of the $\mathrm{H}$ atom and RT is PV for an ideal gas. Thus, eq (122) becomes

$$
E=D H^{\circ}=\left(Z_{A-H}-Z_{A}\right)-5 R T / 2
$$

As an example, consider the process $\mathrm{CH}_{3}-\mathrm{H} \rightarrow \mathrm{CH}_{3}{ }^{*}+\mathrm{H} \cdot$. To estimate the difference in zero point energies between $\mathrm{CH}_{3}-\mathrm{H}_{\text {and }} \mathrm{CH}_{3}{ }^{-}$, I have assumed that one $\mathrm{C}-\mathrm{H}$ stretch of $3100 \mathrm{~cm}^{-1}$ and two $\mathrm{H} \cdot \mathrm{C} \cdot \mathrm{H}$ bends of 1450 $\mathrm{cm}^{-1}$ have been lost in going from $\mathrm{A}-\mathrm{H}$ to $\mathrm{A} \cdot$ and $\mathrm{H} \cdot$. This corresponds to a zero point energy difference of $8.575 \mathrm{kcal}$. For cases like this, the bond energy will be

$$
E c s=D H^{\circ}+8.575-5 R T_{298} / 2=D H+7.095 \mathrm{kcal}
$$

The zero point energy difference for other types of bonds can probably be satisfactorily estimated in a similar manner. Having obtainedf $E b s$ and $E c s$ in this manner we can calculate $P$ and $Q$ from eq (12).

8) Rts, Ets, Beta are the triplet interaction parameters in $\AA$ kcal and $\AA^{-1}$, respectively. I have been using the values given in Johnston [1966], table 11-1. 
9) $F b s, F c s, F p s i 2 s, F p s i 4 s$ are the single bond force constants. The first two are the stretching constants in dynes $/ \mathrm{cm}$; the second two are bending force constants in dyne-cm. In the 3 mass point case, both the bending force constants are set to zero. For 4 point models, only one of the bending force constants will have a value of zero.

10) $S a, S b, S c$ are the partition function symmetries for $\mathrm{A} \cdot \mathrm{H}, \mathrm{B} \cdot$, and $\mathrm{A} \cdot \mathrm{H} \cdot \mathrm{B}$, respectively.

11) Schem is the chemical multiplicity.

12) $S e a, S e b, S e c$ are the electronic degeneracies for A-H, B , and A $\cdots H \cdot B$. Sea will normally have the value one. Since $B \cdot$ and $A \cdot \cdot H \cdot B$ each have an unpaired electron, Seb and Sec will normally have the value two.

\subsection{Output}

BEBO first prints out the input data. It then the following properties of the complex:

1) The potential energy of activation $V^{*}$ in $\mathrm{kcal} / \mathrm{mole}$.

2) The bond orders $n$ and $m$ of the $b$ and $c$ bonds.

3) The bond distances $R b$ and $R c$ in $\AA$.

4) The force constant in the $\varrho$ direction in dynes $/ \mathrm{cm}$ and the angle $\varrho$ makes with the $\mathbf{R b}$ axis on a contour plot like figure 3 .

5) The force constant in the $\sigma$ in dynes/cm, and the angle to the $\mathrm{Rb}$ axis.

6) The force constant in the unstable normal mode direction in dynes/cm, and the angle to the $R b$ axis.

7) The force constant in the stable normal mode direction in dynes/cm, and the angle to the $R b$ axis. Note that the normal mode directions are usually not orthogonal.

8) The stretching force constant matrix $F r$ in dynes $/ \mathrm{cm}$.

9) The equations for transforming back and forth between the normal mode and valence bond coordinates.

10) The bending force constants in dyne-cm.

11) The two stretching frequencies in $\mathrm{cm}^{-1}$.

12) The one to three bending frequencies in $\mathrm{cm}^{-1}$.

13) The zero point energy of the complex in $\mathrm{kcal} / \mathrm{mole}$.

14) The zero point energy of the reactants in $\mathrm{kcal} / \mathrm{mole}$.

15) The zero point energy of the products in $\mathrm{kcal} / \mathrm{mole}$.

16) The Eckart potential function parameters $V 1$ and $V 2$ in $\mathrm{kcal} / \mathrm{mole}$.

17) The reduced mass for tunneling $M_{\mathrm{r}}=$ Mrho.

18) The second two of Johnston and Heicklen's tunneling parameters (see section 2.5).

The program then prints out the rate constants as a function of temperature. Also given at each temperature is the logarithm of the rate constant, the logarithm of the Arrhenius preexponential factor, the activation energy, the tunneling correction factor, and the first of Johnston and Heicklen's tunneling parameters. Since the tunneling algorithm has not been checked outside the parameter ranges used by Johnston and Heicklen, their parameters values are listed to make sure that they are within the proper ranges. The limits are $A 1$ and $A 2=0$ to 20 , and $U^{*}=0$ to 16 .

Finally, there are listed the differences between the calculated values of the logarithm of the rate constant and the values predicted by the least squares fitted Arrhenius equation. This fitted curve is shown by the dotted line on the Arrhenius plot. The fitted Arrhenius parameters are given in the caption ot the plot. On the next two pages there is a sample output for the ethane and methyl radical reaction.

\section{References}

[1] Johnston, Harold S. and Parr, Christopher. Activation energies from bond energies. I. Hydrogen transfer reactions. J. Am. Chem. Soc. 85 (17):2544-2551; September 5, 1963.

[2] Johnston, Harold S. Gas phase reaction rate theory. New York: The Ronald Press; 1966. 362 p.

[3] Mahan, Bruce, H. Activated complex theory of bimolecular reactions. J. Chem. Ed. 51(11):709-711; November 1974. 
[4] Johnston, Harold S. Large tunnelling corrections in chemical reaction rates. Advances in Chemical Physics 3:131-170; 1961.

[5] Sharp, Terry E. and Johnston, Harold S. Hydrogen-deuterium kinetic isotope effect, an experimental and theoretical study over a wide range of temperature. J. Chem. Phys. 37(7):1541-1553; October 1, 1962.

[6] Pauling, Linus. Atomic radii and interatomic distances in metals. J. Am. Chem. Soc. 69(3):542-553; 1947 March.

[7] Herschbach, Dudley R. and Laurie, Victor W. Anharmonic potential constants and their dependence upon bond length. J. Chem. Phys. 35(2):458-463; August 1961.

[8] Johnston, Harold S. Continuity of bond force constants between normal molecules and Lennard-Jones pairs. J. Am. Chem. Soc. 86(8):1643-1645; April 20, 1964.

[9] Gilliom, Richard D. Activation energies from bond energies. A modification. J. Am. Chem. Soc. 99(26):8399-8405; December 21, 1977.

[10] Sato, Shin. On a new method of drawing the potential energy surface. J. Chem. Phys. 23(3):592-593; March 1955.

[11] Herzberg, Gerhard. Molecular spectra and molecular structure I. Spectra of Diatomic Molecules. New York: D. Can Nostrand Company; $1950.658 \mathrm{p}$.

[12] Wilson, E. Bright, Jr., Decius, J. C., and Cross, Paul C. Molecular Vibrations. New York:McGraw-Hill Book Company; 1955. 388 p.

[13] Herschbach, Dudley R., Johnston, Harold S., and Rapp, Donald. Molecular parlition functions in terms of local properties. J. Chem. Phys. 31(6):1652-1661; December 1959.

[14] Herzberg, Gerhard. Molecular spectra and molecular structure II. Infrared and raman spectra of polyatomic molecules. Princeton, New Jersey: D. Van Nonstrand Company, 1945. 632 p.

[15] Hohn, Franz E. Elementary matrix algebra New York: MacMillan Company, 1964.395 p.

[16] Eckart, Carl. The penetration of a potential barrier by electrons. Phys. Rev. 35(11):1303-1309; June 1, 1930.

[17] Johnston, Harold S. and Heicklen, Julian. Tunnelling corrections for unsymmetrical Eckart potential energy barriers. J. Phys. Chem. 66(3):532-533; March 1962.

[18] Abramowitz, Milton and Stegun, Irene A. Handbook of mathematical functions with formulas, graphs, and mathematical tables. Nat. Bur. Stand. (U.S.) Appl. Math. Ser. 55; June 1964. 1046 p.

[19] Arthur, N. L., Donchi, K. F., and McDonnell, J. A. BEBO calculations. III. A new triplet repulsion energy term. J. Chem. Phys. 62(4):1585-1586; February 15, 1975.

[20] Hirschfelder, J. 0. and Linnett, J. W. The energy of interaction between two hydrogen atoms. J. Chem. Phys. 18(1):130-142; January 1950.

\section{APPENDIX: Various Triplet Functions}

The subroutine Trpl is able to provide three different triplet functions which are selected according to the value of flag $0 \mathrm{pt}(0)$. They are as follows:

$\mathrm{Opt}(0)=0$ : This is the modified Sato triplet function with a small portion neglected. Instead of Eq. (13), V. $=\mathrm{E}_{t s}$ is used. This simpler formula seems to have been used in the days of mechanical desk calculators. This option is useful when attempts are being made to reproduce the results of earlier workers.

$0 p t(0)=1:$ Eq. $(13)$ is used.

$\mathrm{Opt}(0)=2$ : Arthur et. al. [19] have developed a triplet energy formula by fitting a function to the $\mathrm{H}-\mathrm{H}$ triplet potential energy values given by Hirschfelder and Linnett [20]. Their formula is

$$
V_{t}=5.873 E_{t s} e^{-1.747 \beta_{\left(R_{b}+R_{c}\right)}\left(\beta\left(R_{b}+R_{c}\right)\right)^{1.525}}
$$

They claim better results in certain cases when this function is used. 
Fun ldentific

$\mathrm{CHSCHZ-H}+\mathrm{EH} 3=\mathrm{CHOCH}+\mathrm{H}-\mathrm{CH} 3$

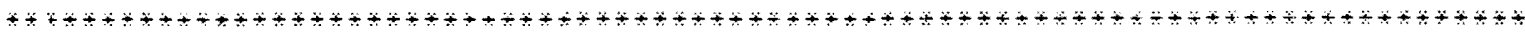

Opt ions UEed in Calsul at ions:

Modified sato triplet function.

Five-point difference formul as used to get actuation Energy.

Esag 10 logarithm of the $r$ ate constant.

Fite constant unit $s$ in 1 it $e r s<m o l e-5$.

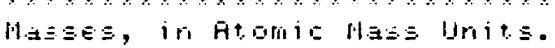
$M 1=17.0510$
$M 2 \approx 12.0119, \quad 43=$
1. 0050
$144=12.0115$
$195=$
3.946

Single Eond Il izt aneEz, in Angztroms.

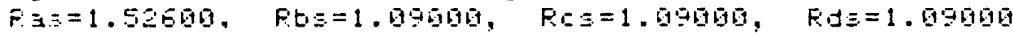

Single Eand Energies of Center Eonds, in kcal; alsop a q F ameters. $E b s=155.105, E: z=111.150, p=1.085, \quad q=1.093$

Single Eond Enspgy, Ilistance, a Mor se Farameter for Triplet Interaction.

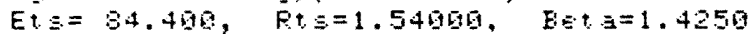

Single Eond Stretching Fores Constants in dynesen for Eenter Eonds. $F b=4.79000 E+05, F s=4.79000 E+05$

Single Eand Eending Force constants in dyne-cm for Duter Maszes. $F p \equiv 125=9.14387 E-12, \quad F p \equiv i 45=5.4553 Q E-12$

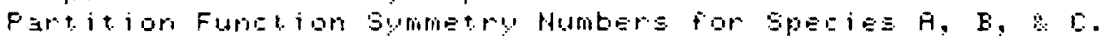
$S A=1, \quad S E=1, \quad S C=1$

Eremical Multiplicity. Sinent $=E$

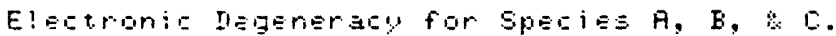
$S E F=1, \quad S E E=2, \quad S E C=2$

Pauling E Eond-order Parameter. $L$ Lind $\equiv=0.2800$

Froperties of Compley.

Potantial Energy of Het. uation: $\because=14.596 \mathrm{kcal}$

Eorid Order Far anetera: $H=0.5804, M=0.4190$

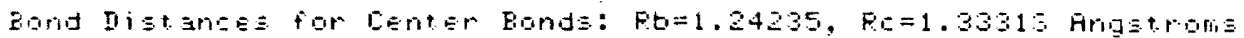

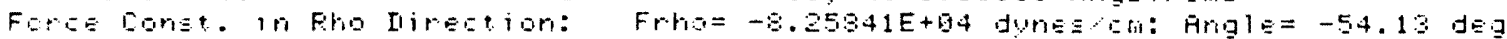

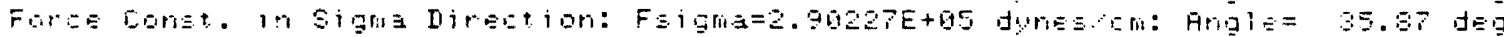

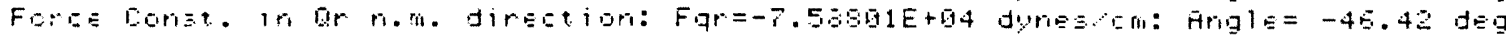

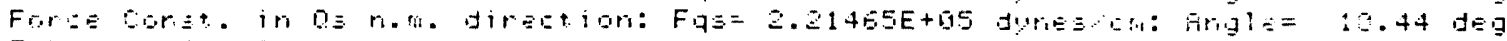

F liatrix for stretchez in dunesar
1. $E 2 Z 2+E+05$

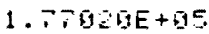
1.77020E+05
$4.54188 E+94$

Horna! Eacrdinate Transformat ionj

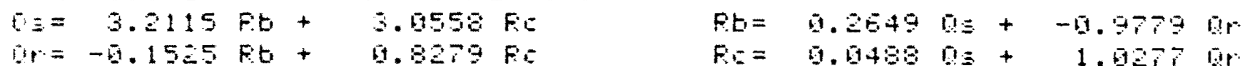

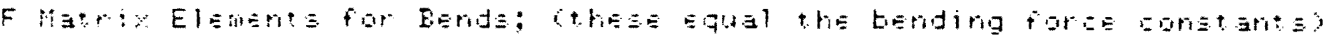

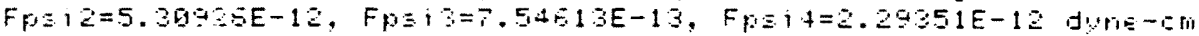

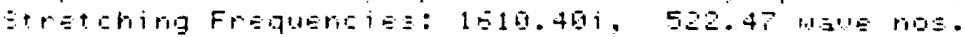

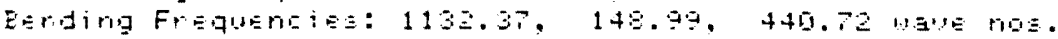

Zero Foint Energy of Eoraplex= 5.50\% ba

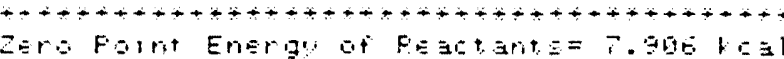

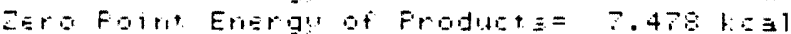

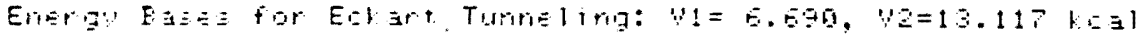

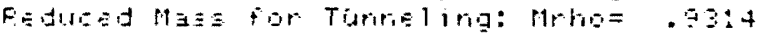

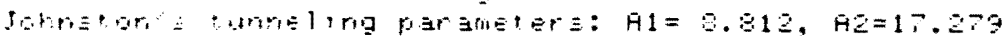

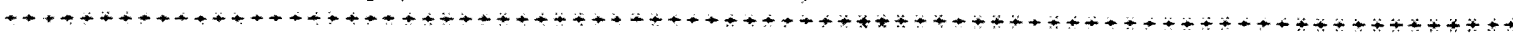




\begin{tabular}{|c|c|c|c|c|c|c|c|c|c|c|}
\hline Emp., & Rat: & $\operatorname{con}=1, \ldots$ & factor, & ACt. Enet & पy, Eckart. & $U * F$ & actar, & Deviation & from & Fit \\
\hline $1000<\mathrm{T}$ & $T$ & $r$ & $\log (k)$ & LOg(A) & Eact & 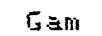 & $U F$ & Fit. & & \\
\hline 6.50 & 2005 & $1.795 E+09$ & 9.254 & 11.724 & 22.597 & 1.041 & 1.209 & -5.518 & & \\
\hline 0.78 & 1286 & 1. $030 E+08$ & 8.013 & 11.179 & 18.626 & 1.591 & 1.867 & 0.019 & & \\
\hline i. 96 & 947 & $5.106 E+06$ & 6.959 & 10.718 & 16.294 & 1.166 & 2.534 & 0.017 & & \\
\hline 1.33 & 750 & $1.036 E+06$ & 6.015 & 10.347 & 14.566 & 1.273 & 3.260 & 0.005 & & \\
\hline $1 . E 1$ & 621 & $1.396 E+65$ & 5.145 & 10.022 & 13.850 & 1.422 & 3.867 & -0.006 & & \\
\hline 1.89 & 529 & 2. $129 E+194$ & 4.328 & 9.727 & 13.979 & 1.527 & 4.534 & -9.013 & & \\
\hline 2.17 & 462 & $3.577 E+93$ & 3.553 & 9.454 & 12.462 & 1.909 & 5.201 & -0.016 & & \\
\hline 2.44 & 409 & $6.501 E+02$ & 2.813 & 9.193 & 11.943 & 2.300 & 5.867 & -0.011 & & \\
\hline 2.72 & 367 & $1.254 E+02$ & 2.102 & 8.944 & 11.502 & 2.851 & 6.534 & 0.091 & & \\
\hline 3.00 & 333 & $2.599 E+11$ & 1.415 & 8.716 & 11.135 & 3.627 & 7.201 & A. 020 & & \\
\hline
\end{tabular}

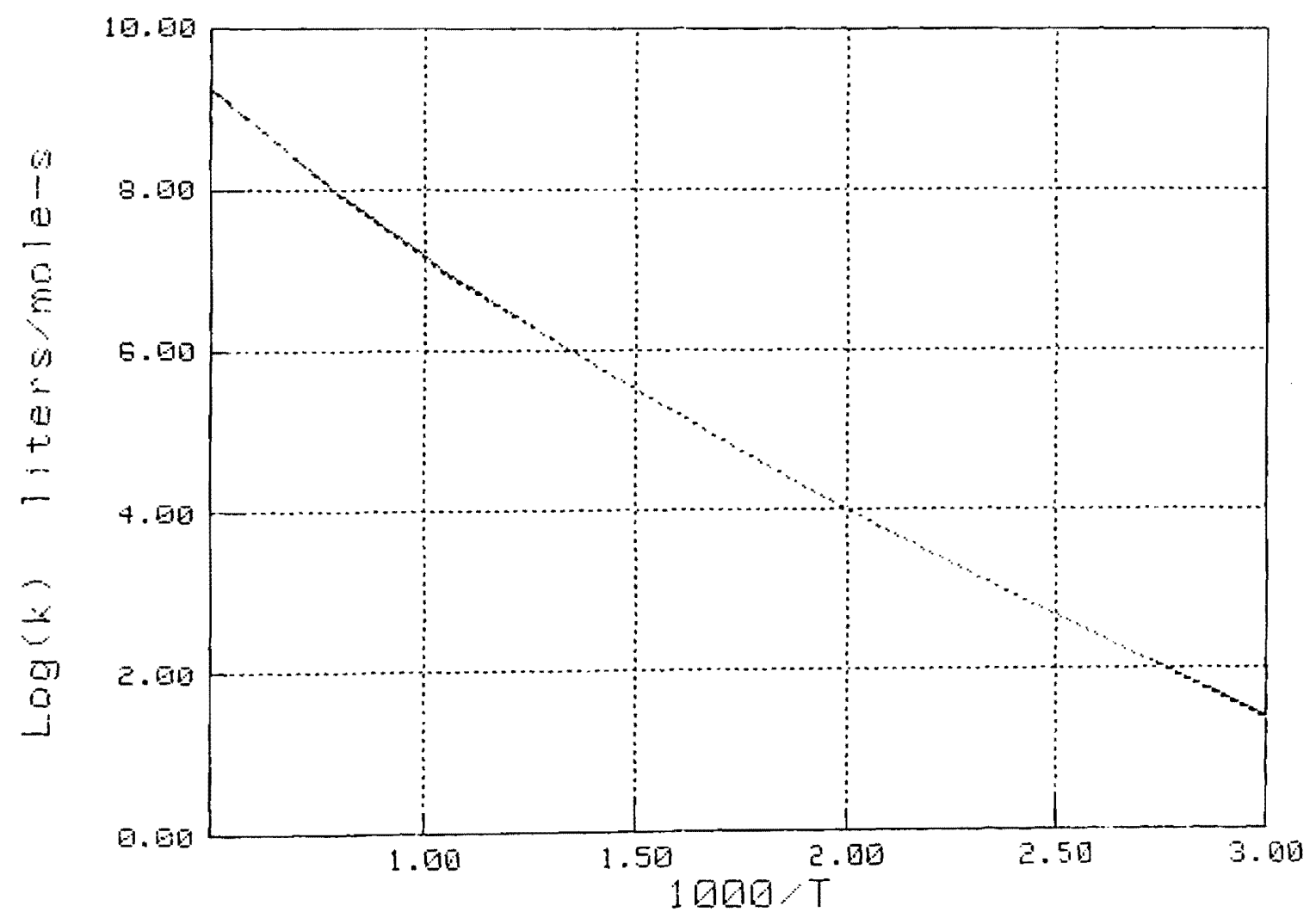

Figure 1. Plot of logarithm of rate conetant as a function of the reciprocal of the temperatide.

A leset aquares fit of the calculated rate constant to the Arrherius expression, $k=A *(T \cap n) * E X P$-Ear R RT), yields the following values for the paratisters:

$\log (A)=-2.3 E 2 E+0 B, \quad n=3.821, \quad E a r r=3.974$ 
This is a listing of the computer program for calculating the rate constants of hydrogen atom transfer reactions according to the bond-energy-bond-order method fustomarichere language for use on Hewlett-Packard $9845 A$ computer.

OVERLAP

READ H, K, Lam, Pmass, Econv, Prac1, Imax,, $\mathrm{NuLL}$

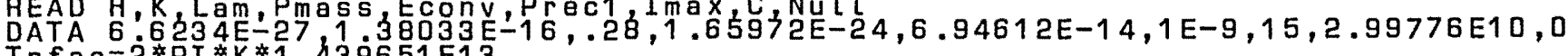
Tpfac $=2 * P I * K * 1.439651 \mathrm{E} 13$

DIM Runid $\$ 79]$

DIM Opt (7) Temp $(1: 16)$ Tpktkcal $(1: 16), K t k c a L(1: 16), K q p r(1: 16), \operatorname{Eactpr}(1: 16)$

DIM Gampr\{1:16\}, Lkqprl1:16\}, Lapr\{1:16\}, Ustr\{1:16\},Error $\{1: 16\}, P a r\{1: 3\}$

COM DeLta, Lam, Nbebo, Rbs,Rcs, Rts, Betab,Betac,Beta,Ebs, Ecs, Ets

READ Runjd\$. IRun identification.

READ Opt[D],Opt(1),Opt (2),Opt (3),Opt (4),Opt (5), Opt (6), Opt (7)

READ $\mathrm{N}$ temp

IF N temp $\angle=16$ THEN L62

PRINT "Error-03"

BEEP

62: IF $N$ temp<5 THEN Opt $(1)=2$

REDIM Temp (1:Ntemp),Tpktkcal $[1: N t e m p), K t k c a l(1: N t e m p), K q p r(1: N t e m p)$

REDIM Eactpri. N IF N temp=1 THEN REA tmax

IF N temp=1 THEN Tm $i n=1$

IF $N$ temp $=1$ THEN Opt $[1]=2$

IF Ntemp $>1$ THEN READ Tmin, Tmax lThe minimum and maximum temperatures.

Rtm $\mathrm{in}=1000 / \mathrm{Tmax}$

Rtmax=1000/Tmin

FOR $L=1$ TO $N$ temp

Temp $[L]=1000 /[\mathrm{Rtmin+[L-1)*DeLrt]}$

Tpktkcal $[L]=T$ p f a ${ }^{*}$ Temp $(L)$

ktkcal[L]=Tpktkcal(L\}) $(2 * P I)$

NEXT

Delrt $=$ Delrt/1000

READ $M 1, M 2, M 3, M 4, M 5$

IF $M 1>0$ THEN MU $1=1 / M 1$

IF M5>0 THEN MU $5=1 / M 5$

READ Ras, Rbs, Rcs, Rds

IF $R$ as $>0$ THEN Rhoa $=1 /$ Ras

IF Rds $>0$ THEN Rhod=1/Rds

READ Ebs, Ecs, P,Q

READ Rts, Ets, Beta

READ Fbs,Fcs,Fpsi2s,Fpsi4s

I Atomic mass units.

READ $\mathrm{Sa}, \mathrm{Sb}, \mathrm{Sc}$

READ Schem

READ Sea Seb Sec

PAINTER IS 16

IAngstroms.

lEnergies in $\mathrm{kcal} / \mathrm{mole}$.

IStretching constants in dynes/cm.

Bending constants in dynes-cm.

Partition function symetries.

Chemical multiplicity.

IElectronic degeneracy. 
PRINT "Run Identification:"

作 $j$ ons Used in Calculations:"

IF opt

IF Opt 0 ( $)=2$ THEN PRINT "TwO-parameter Arthur et.al. to t

580

IF OPt $[0]=3$ THEN PRIN

triplet function."

590

6: "IF Opt $(1)=1$ THEN PRINT "Five-point difference formulas used to get activation energ

PRINT "Three-parameter Arthur et.al. style triplet function."

600

610

620

630

640

650

660

670

680

IF $p_{1} t(1)=2$ THEN PRINT "Three-point difference formulas used to get the activation energ IF ${ }^{y}$ D $(2)=1$ THEN PRINT "Natural Logarithm of the rate constant."

IF opt (2)=2 THEN PRINT "Base 10 Logarithm of the rate constant:"

IF opt $\{4\}=1$ THEN PRINT "Rate constant units in cc/mole-s."

IF Opt (4)=2 THEN PRINT "Rate constant units in cc/molecuie-s."

IF Opt 4$\}=3$ THEN PRINT "Rate constant units in liters/mole-s."

IF Opt $4=4$ THEN PRINT "Rate constant units in liters/molecule-s."

IF Opt

PRINT "Masses, in Atomic Mass Units."

PRINT USING Fórmat $31, M 1, M 2, M 3, M 4, M 5$

710 Format31: D

PRINT "Single Bond Distances, in Angstroms"

730 PRINT USING Format32;Ras, Rbs; Rcs, Rds

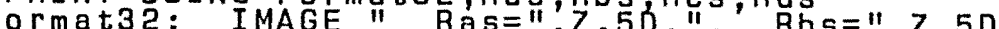

PRINT "Single Bond Energies of Kenter Bon, ", Rcs=",Z.5D," Rds=",Z.5D

PAINT USING Format33; Ebs.ECs P

770 Format33: IMAGE" "Ebs=",3D,30," Ecs="3D.3D,", $p=", z, 30, ", q=", z, 30$

PRINT "Single Bond Energy Distance, \& Morse Parametér for Triplet'Interaction."

PAINT USING Format34;Ets, Rts, Beta

Format34: IMAGE "Ets=",3D,3D,", Rts=",Z.5D,", Beta=",Z.4D

PR INT "Single Bond Stretching Force Constants'in dynes/cm for Center Bonds."

PRINSING Format35;Fbs, FCs

Prmat35: IMAGE " Fbs=",Z.5DE,", Fcs=", Z.5DE

PRINT "Single Bond Bending Force Constants in dyne-cm for Outer Masses."

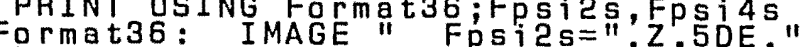

"PिT "Pasi4s=", Z .5DE

PRINT USING Form

Format37: IMAGE " SA=",

PRINT "chemical Multiplicity." $\mathrm{SB}=", \mathrm{Z}, ", \quad \mathrm{SC}=", \mathrm{Z}$

PRINT USING Format3G; Schem

920 PRINT "ELectronic Degeneracy for Species $A, B, \& C . "$ 
940 Format39: IMAGE " SeA=",Z," $\begin{gathered}S e B=", Z, ", \quad S e C=", Z \\ 950\end{gathered}$

960 PRINT USING Format $41 ;$ Lam

970 Format 41. IMAGE " Lamda=" Z 40

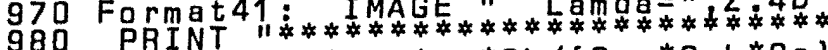

990 Sym=Schem*Sec*Sa*Sb/[Sea*Seb*Sc)

1000

$\mathrm{Fb} s=1 \mathrm{E}-16 * \mathrm{Fbs} / \mathrm{E}$ conv

Force constants are converted to kcal energy units.

1010 FCS=1E-16*FCs/Econv

1020 Fpsi2s=Fpsi2s/Econv

1030

1040

1050

$4 s=F s^{2} 4 \mathrm{~s} / \mathrm{E}$

The saddle point position is calculated.

1070 I count $=0$

1090

1100

1101

1110

1120

IF

No $L d=N$

$C A L L=1-N$

$M=1-N$

$1130 V=E b s^{*}\left(1-N^{*} P\right)-E C s^{*} M^{*} Q+V t$

$1140 \quad V n=-E b s * P * N *[P-1]+E c s * Q * M *(Q-1)+V t n$

$1150 \quad V n n=-E b s * P *[P-1\} * N *\{P-2]-E \operatorname{cs} * Q *(Q-1) * M-(Q-2)+V \tan$

$1160 N=N o L d-V n / V n n$

1170 IF ABS[ [N-NoLd]/NoLd]<Prec1 THEN L10

O 1180 Icount=Icount +1

\& 1190 IF I count>Imax THEN L12

1200 GOTO L11

12: PRINT "Error-01"

1220 STOP

10. $N=N b$ ebo $=N o L d$

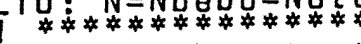

I $\mathrm{Next}$

$N s q=N^{2}-2$

$1270 \mathrm{Msq}=\mathrm{M}^{-}$

$1280 \quad N 2 m 2=N s q+M s q$

$1290 \quad \mathrm{Nm}=N * M$

$1300 \mathrm{Frho}=V n n^{*} \mathrm{Nm}^{\star} 2 /\left[\mathrm{N} 2 \mathrm{~m} 2 * \mathrm{Lam}^{\star} 2\right]$

$1310 \mathrm{Fsigma}=\left(\mathrm{Fbs}^{*} * \mathrm{~N} \wedge \mathrm{G}+\mathrm{Fcs} * \mathrm{M} * \mathrm{3}+\mathrm{Vtr} \mathrm{r}\right) / \mathrm{N} 2 \mathrm{~m} 2$

130 DIM Fr $(1: 2,1: 2)$

1320 DIM Fr 1 : $2,1: 2)$ F $+F s i g m a * N s q) / N 2 m 2$

$1330 \operatorname{Fr}\{1,1\}=[F r h o * M s q+F s i g m a * N s q\} / N 2 m 2 / N 2 m 2$

1350 Fr $\{2,2\}=\{F r h o * N s q+F s i g m a * M s q\} / N 2 m 2$

$1360 R b=R b s-L a m * L O G(N)$

1380 D $r=D E T\{F r$ \}

3 90 of $r=S G N(D f r) * S O R$ (ABS (Dfr)]

$1400 \mathrm{Jac}=\left[\mathrm{Rb} * \mathrm{Rc} / \mathrm{Rb} \mathrm{f}^{*} \div 2\right.$

1410 Rho $b=1 / R b$

1420 Rhoc $=1 / \mathrm{RC}$

$1430 \mathrm{CC}=-\mathrm{Nbebo} /\{1-\mathrm{Nb} \mathrm{Bbo}\}$ 
$1440 \quad M a=M 1+M 2$

$1450 M x=M 3$

$1460 \quad M b=M 4+M 5$

$1461 M a \times b=M a+M x+M b$

$1470 M r h o=\left(M a * M b *(1+C c)-2+M b * M x^{*} C c^{\wedge} 2+M a * M x\right) /\left(M a \times b *\left(1+C c^{*} 2\right)\right)$

1471 DIM Azr $\{1: 3,1: 2\}$ ALq $(1: 3,1: 2\}$

1472 Azr $\{1,1\}=-(M b+M \times\}) M a \times b$

1473 Azr 1,2$\}=A z r\{2,2\}=-M b / M a x b$

147 Azr $2,1,=A z r\{3,1\}=M a / M a x b$

$1475 A z r(3,2)=(M a+M x) / M a x b$

1480 Mrho=1

1490

1500

1510

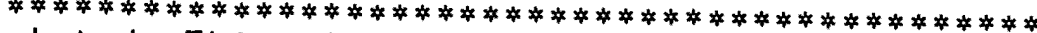

wext, the Gr matrix is calculated. This will hold for $3,4,45$ atom models

1520

1530

1540

1550

1570

1580

oIM Grif the type of model being used is determined. (Note, all are linear) Model $=5$

IF $\{M 1=0\}$ OR $(M 5=0)$ THEN Model $=4$

Mudel $=$ Model -2

$1590 \mathrm{Mu}=1 / M$

1600 Mu $4=1 / M 4$

o $\quad 1610 \mathrm{Gr}(1,1)=M u 2 /(1+M 1 / M 2)+M u 3$

$1620 G r(1,2)=G r[2,1]=-M u 3$

$1630 \mathrm{Gr}\{2,2\}=M u 3+M u 4 /(1+M 5 / M 4)$

1650 DIM Hriching frequencies evaluated.

1650 DIM Hr $[1: 2,1: 2]$

1660 MAT $H r=G r^{*} F r$

1670 B $\mathrm{B}=\mathrm{Hr}(1,1\}+\mathrm{Hr}\{2,2\}$

$1690 \mathrm{Dh}=\mathrm{SQR}(B \mathrm{~B} * 2-4 * \mathrm{Ch})-\mathrm{Hr}(1,2) * \mathrm{Hr}(2,1)$

1700 DIM Evs $(1: 2), F r q s[1: 2], L s[1: 2)$

1710 Evs $(1)=.5 *(B h+D h)$

1720 EVs 12$\}=5 * 5 *[B h-D h\}$

NEXT I I

1790 DIM G $(1: 3,1: 3], F[1: 3,1: 3], H(1: 3,1: 3], E y b[1: 3), F r g b[1: 3], L b(1: 3)$

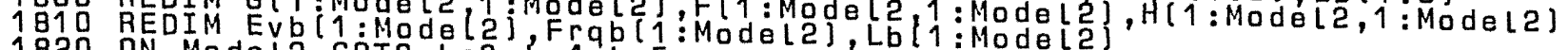

1820 DN Modale GoTo La3, La4, La5 


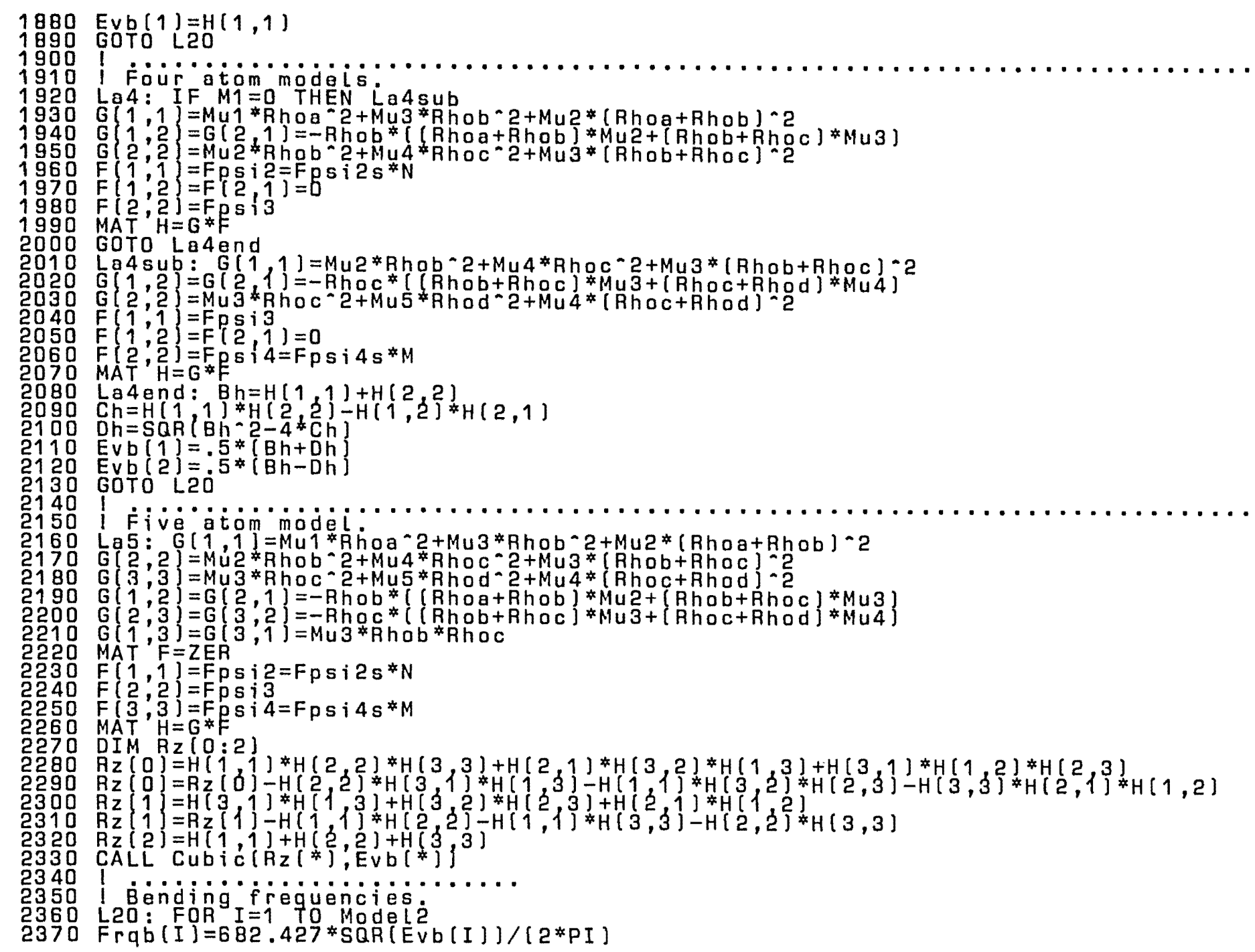


2380 NEXT I

2390 PRINT "Properties of Complex."

2400 PRINT USING Format1;V

2410 Format1: IMAGE "Potential Energy of Activation: V=",DD.3D," kcal"

2420 PRINT USING Format2;N,M

2430 Format2: IMAGE "Bond órder Parameters: $N=", Z .4 D, ", M=", Z .4 D$

2440 PRINT USING Format3;Rb R

2450 Format3: IMAGE "Bond Distances for Center Bonds: Rb=",Z.5D, ", Rc=",Z.5D," Angstroms"

2460 PRINT USING Format4;Frho*Econv*1E16, ATN[Cc]*180/PI

2470 Format4: IMAGE "Force Const. in Hho Direction: Frho= ",MZ.5DE," dynes/cm:"," Angle=", M DDZ.DD" deg"

2480 PRINT USING Format5;Fsigma*Econv*1E16, ATN $(-1 / C c) * 180 / P I$

2490 Format5: IMAGE "Force Const. in Sigma Direction: Fsigma=",Z.5DE," dynes/cm:"," AngLe=",M DDZ.DD" deg"

2491 DIM Lgi $(1: 2,1: 2\}, \operatorname{Lq}[1: 2,1: 2\}), L q i(*))$

2492 CALL NornOd Hri

2494 DEF FNGg $[Z]=\operatorname{Fr}[1,1] * \operatorname{COS}[Z] * 2+2 * F r[1,2] * \operatorname{SIN}[Z) * \operatorname{Cos}[Z]+F r(2,2] * \operatorname{SIN}(Z) * 2$

$2495 Z=\operatorname{Lq}(2,2\} / \operatorname{Lq}\{1,2\}$

2496 Zr=ATTNZZ\}

2500 PRINT USING Format $4 a ; F N G g[Z r] * E c o n v * 1 E 16, Z$ Z $180 / P I$

2510 Format4a: IMAGE "Force Const. in Qr n.m. "direction

$2511 Z=\operatorname{Lq}[2 ; 1\}) \operatorname{Lq}[1,1]$

Q 2512 Zs=ATTN(Z)

2520 PRINT USING Format4b;FNGg[Zs]*Econv*1E16,Zs*180/PI

2530 Format4b: IMAGE "Force Const. in Qs n.m." direction: Fqs=",MZ.5DE,"dynes/cm:"," Angle=",

2531 MAT ALP =AZ* deg

2532 I MAT PRINT A

2533 DIM Rcomplex[1:2̉, Zeq $(1: 3)$

534 Rcomplex 11$\}=$ Rb

2535 Rcomplex $\{2\}=R c$

2536 MAT $Z$ eg=Azr* Rcomplex

2537 I MAT PRINT Zeq

2540 PRINT "F Matrix for Stretches in dynes/cm"

2550 PRINT USING "2X, MZ.5DE, $2 X^{\prime \prime} ; F r(1,1) * E \operatorname{conv} * 1 E 16, F r(1,2) * E \operatorname{conv} * 1 \mathrm{~F}_{16}$

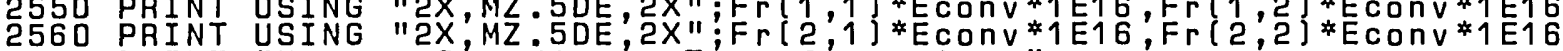

2600 PRINT "Normal Coordinate Transformations"

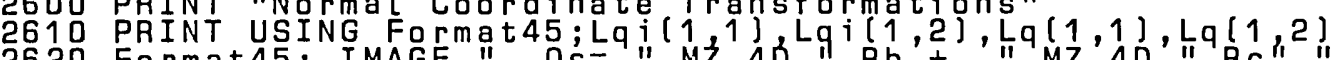

2620 Format45: IMAGE " QS= ", MZ.4D," Rb + ", MZ:4D," AC"," $4{ }^{4}$ "UC" USING Format46; Lqi $(2,1), \operatorname{Lq} i[2,2], \operatorname{Lq}(2,1), \operatorname{Lq}[2,2]$

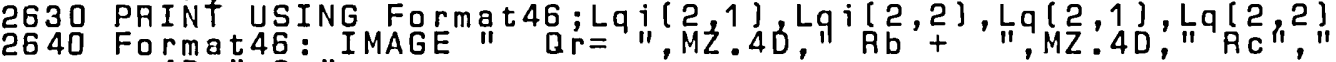
$4 D$ "Q $r$ "

2650 PRINf "F Matrix Elements for Bends; [these equal the ben

2660 PRINT USING Format6;Fpsi ${ }^{*}$ Econv, Fpsi $3 *$ Econv Fpsi 4 * Econv

2670 Format6: IMAGE " Fpsi2=" Z 5DE," Fpsi3=",Z.5DE,", Fpsi4=",Z.5DE,"dyne-cm"

2680 PRINT USING Format7; -Frqs $\{2\}$,Frqs 11$]$

2690 Format7: IMAGE "Strétching Frequencies: ",4D.DD, "i, ",4D.DD," wave nos." 


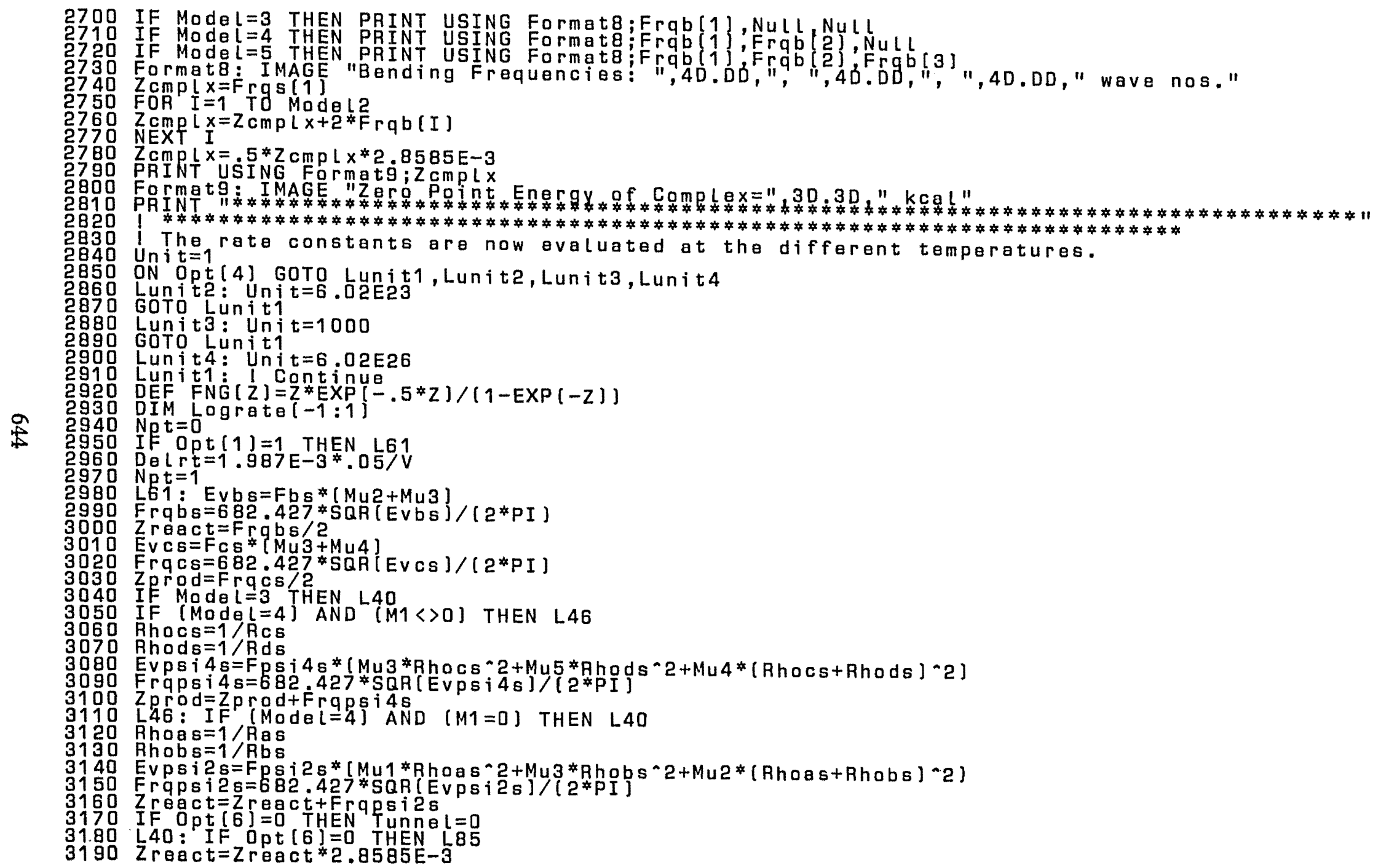

IF Opt (1) $=1$ THEN L6 1

DeLrt $=1.987 E-3^{*} .05 \% \mathrm{~V}$

$\mathrm{Npt}=1$ 
3200 Zprod=Zprod*2.8585E-3

3210 PAINT USING Format10;2ract

32ट Format10: IMAGE "Zero Point

3 PRINT USING Farmat11;Zprod

3240 Format11: IMAGE "Zerópoin

$3250 \quad V 1=V-Z$ react

3260 Tunnel=1

3270 IF V $1>0$ THEN LB 4

3280 Tunne $L=0$

PRINT "graater than the

GoT0 L85

L84: V $2=V-E b s+E c s-Z$ prod

PRINT USING Format12;V1, v2

PRINT USING Formati3;

Formati3: IMAGE "Reduced Mass far Tunneling: Mrho=",DD.4D

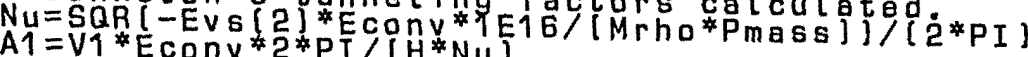

$A Z=V Z * E \operatorname{conv} * 2 * P I /[H * N u)$

3402 Tunne $L=0$

3410 L3DO: PRINT USING Format16;A1, A2

Eckart tunneling factor not

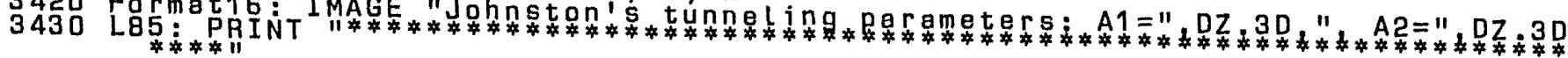

3440 IF $D P t[3]=0$ THEN PRINTER IS 16

3460 PRINT $=1$ TO Ntemp

3460 PRINT USING Format20;L, Ntemp

Format20: IMAGE "Calculating rate const. at ",DD,"th temperature value out of ",DD," va

3480 Rtemp=i/Temp(L)

3490 FOR Nt $=-N p t$ To Npt

$3500 R t=R t e m p+N t * D e l r t$

$3510 \mathrm{~T}=1 / \mathrm{R}$

3520 Tpktkcalsub=Tpfac*T

3530 Ktkcalsub=Tpktkcalsub/ (2*PI)

3540 Tfac=EXP $(-V / K t k c a l s u b) * S y m * J f a c * C * F r q s(2) * S Q A(F b s) /(D f r * U n i t)$

3560 ON MOd

3570 Lb3: K L =K

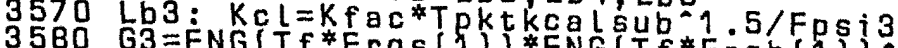

$3590 \mathrm{Kg}=\mathrm{Ga} * \mathrm{~K}$ (Frqs $(1)\} * F N G(T f * F r q b(1)\} * 2 / F N G(T f * F r q b s]$

3600 GOTO 30 C

3610 Lb4: IF M1 =0 THEN Lb4sUb

3620
3630
G4 
$3640 \quad G 4 a=64 a /(F N G(T f * F r q b s) * F N G(T f * F r q p s i 2 s) * 2)$

$3650 \quad \mathrm{Kg}=\mathrm{G4} \mathrm{a}^{*} \mathrm{KC} \mathrm{L}$

3660 GOTO LOD

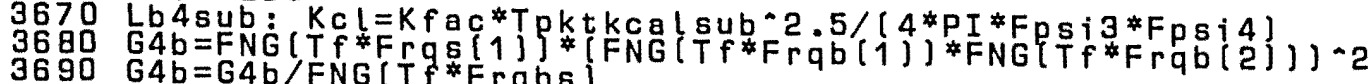

3690

$3700 \quad K g=G 4 b * K c L$

3720 Lbs: Kcl=Kfac*Tpktkcalsub*2.5*Fpsi2s / (4*PI*Fpsi2*Fpsi3*Fpsi 4)

3740

3750

3760

3760

3781

3790

3800

3810

3820

3830

3840

3860

$G 5=G 5$ / (FNG (Tf*Frqbs]*FNG [Tf*Fqqusi2s]

$\mathrm{Kq}=\mathrm{G} 5 * \mathrm{Kcl}$

L3O: $\mathrm{Gam}=1$

IF Tunnel=0 THEN L87

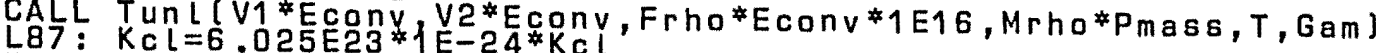

$\mathrm{Kq}=6.025 \mathrm{E} 23 * 1 \mathrm{E}-24 * \mathrm{Kg} * \mathrm{Gam}$

IF $N t=0$ THEN $G$ T $t a b=K a$

IF $N$ t $=0$ THEN Gamtab
Lograta $(N \mathrm{~N})=L O G(K q)$

NEXT $N t$

Eactpr $\{(L)=-1.987 E-3 *\{-\operatorname{Lgg} r a t e(-1)+\operatorname{Lggrate}(1)\} /(2 * D e L r t)$

3890 L59: KgPr (L) $=K a t a b$

3900

3910

3920

3930

IF Opt $\{2\}=1$ THEN

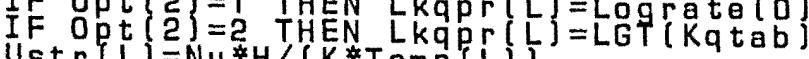

Gampr $[L]=$ Gamtab

3940 NEXT

3950 IF Opt $(3)=0$ THEN PRINTER IS 0

3960 IF NPt=1 THEN L60

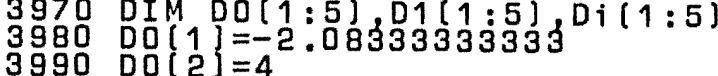

4000 DO 3$\}=-3$

4010 DO $(4)=1$. 33333333333

4020 DO $(5)=-.25$

4040 D $1\{2\}=-.833333333333$

4040

4050

4060 D $1(4)=-.5$

$4070 \mathrm{D} 1(5)=8.33333333333 \mathrm{E}-2$

$4080 \mathrm{Di}(1)=8.33333333333 \mathrm{E}-2$

4090 Di (2) $=-.666666666667$

4100 Di 3$\}=0$

4110 Di $(4)=.666666666667$

$4120 \mathrm{D} i[5]=-8.33333333333 \mathrm{E}-2$

4130 MAT Eactpr=ZER 
4140 FOR $J=1$ TO 5

4150 Eactpr $(1)=E a c t p r(1)+D O(J) * \operatorname{Lkgpr}(J)$

4160 Eactpr $\{2\}=E a c t p r\{2\}+D 1\{j\} *$ Lkqpr $\{J\}$

4170 Eactpr (Ntemp-1)=Eacter (Ntemp-1\}-D1 (J)*LKgpr (Ntemp-J+1)

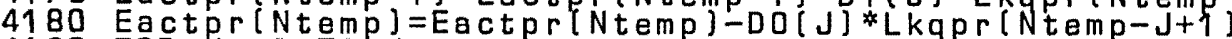

4190 FOR $\mathrm{Na}=3$ TO $\mathrm{Ntemp-2}$

4200 Eactpr $[\mathrm{Na}]=E a c t p r[\mathrm{Na}]+D i(J) * L k q p r[\mathrm{Na} J J-3]$

4210 NEXT Na

4220 NEXT $J$

4230 IF Opt $\{2]=1$ THEN MAT Eactpr=Eactpr* $[-1.987 E-3 / 0$ el $r\}$

4240 IF Opt $[2]=2$ THEN MAT Eactpr=Eactpr*\{-1:987E-3*LOG $[10\} / D e[r t]$

4250 L60: FOR $L=1$ TO N Temp

4260 Afac=Kapr [L]* EXP [Eactpr [L]/KtKcaL[L])

4270 IF Opt $[2]=1$ THEN Lapr $\{L\}=L O G$ (A fac $)$

4280 IF Opt $\{2\}=2$ THEN Lapr $\{L\}=L G T\{$ A fac

4290 NEXT L

4300 IF $[0 \mathrm{pt}(3)=0]$ AND (Ntemp>1) THEN PRINT PAGE

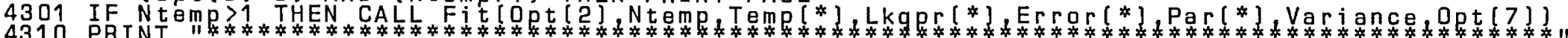

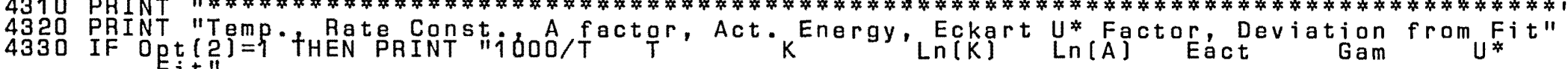

4340 IF Opt $[2]=2$ THEN PRINT "1000/T $T \quad K \quad$ Log(K) Log(A) Eact

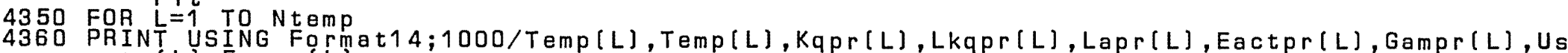
tril $[L], E r$ ror $[L]$

O 4370 NEXT $L$

4380 Format14: IMAGE $1 \mathrm{X}, \mathrm{Z}, \mathrm{DD}, 2 \mathrm{X}, 4 \mathrm{D}, 2 \mathrm{X}, \mathrm{Z}, 3 \mathrm{DE}, 6(1 \mathrm{X}, \mathrm{MDZ}, 3 \mathrm{D})$

4400 IF N temp=1 THEN L63

4401 IF Opt[3]=1 THEN PAUSE

4410 CALL P [ tk[Opt[*], Ntemp, Temp [*], Lkqpr [*],Error[*])

4411 IF $p t[3]=1$ THEN PAUSE

4430 EXIT GRAPHICS

4430 EXIT GRAPHICS

4440 PRINT "Figure 1. Plot of Logarithm of rate constant as a function of the reciprocal of"

4441 PRINT "the temperature."

4442 PRINT "A Least squares fit of the calculated rate constant to the Arrhenius expression,"

4443 IF Opt $[7]=2$ THEN PRINT "k=A*EXP(-Earr/RT), yields the following values for the parameter

4444 IF Dpt (7)=3 THEN PRINT "k=A* $\left(T^{*} n\right) * E X P(-E a r r / R T)$, yields the following values for the par

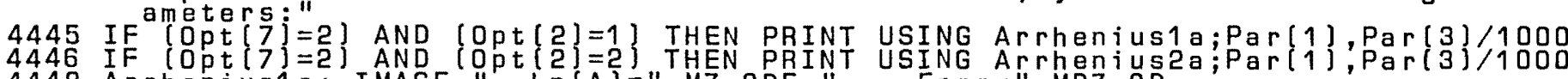

4448 Arrhenius1a: IMAGE " $\operatorname{Ln}(A)=" M Z 3 D E "$ " Earr=" MDZ.3D

4449 Arrhenius2a: IMAGE " LOG(A)="MZ.

4453 IF (OPt (7)=3) AND 10pt $\{2\}=1\}$ THEN PRINT USING Arrhenius1b; Par $\{1\}, P a r\{2\}, P a r\{3\} / 1000$

4457 Arrheniusib: IMAGE " $\operatorname{Ln}(A)=", M Z$. 3DE,", n=",MZ. 3D,",

4458 Arrhenius2b: IMAGE " $\log (A)=", M Z .3 D E, ", \quad n=", M Z .3 D, ", \quad E a r r=", M D Z .3 D$

4460 L63: PRINTER IS 16

4470 IF ODt $[5]=0$ THEN LB2

4480 ! Position of normal coordinate plot.

4490 L82: PRINTER IS 16

4500 SERIAL

4510 END 


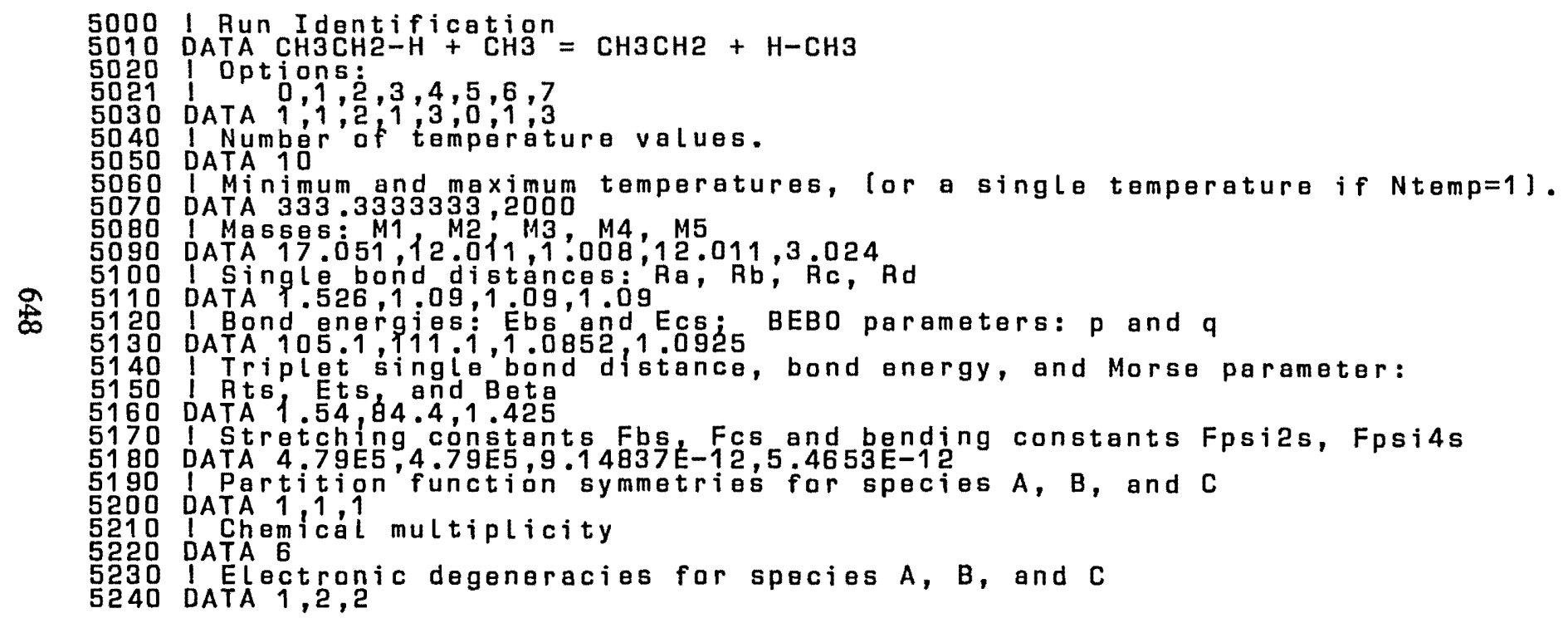




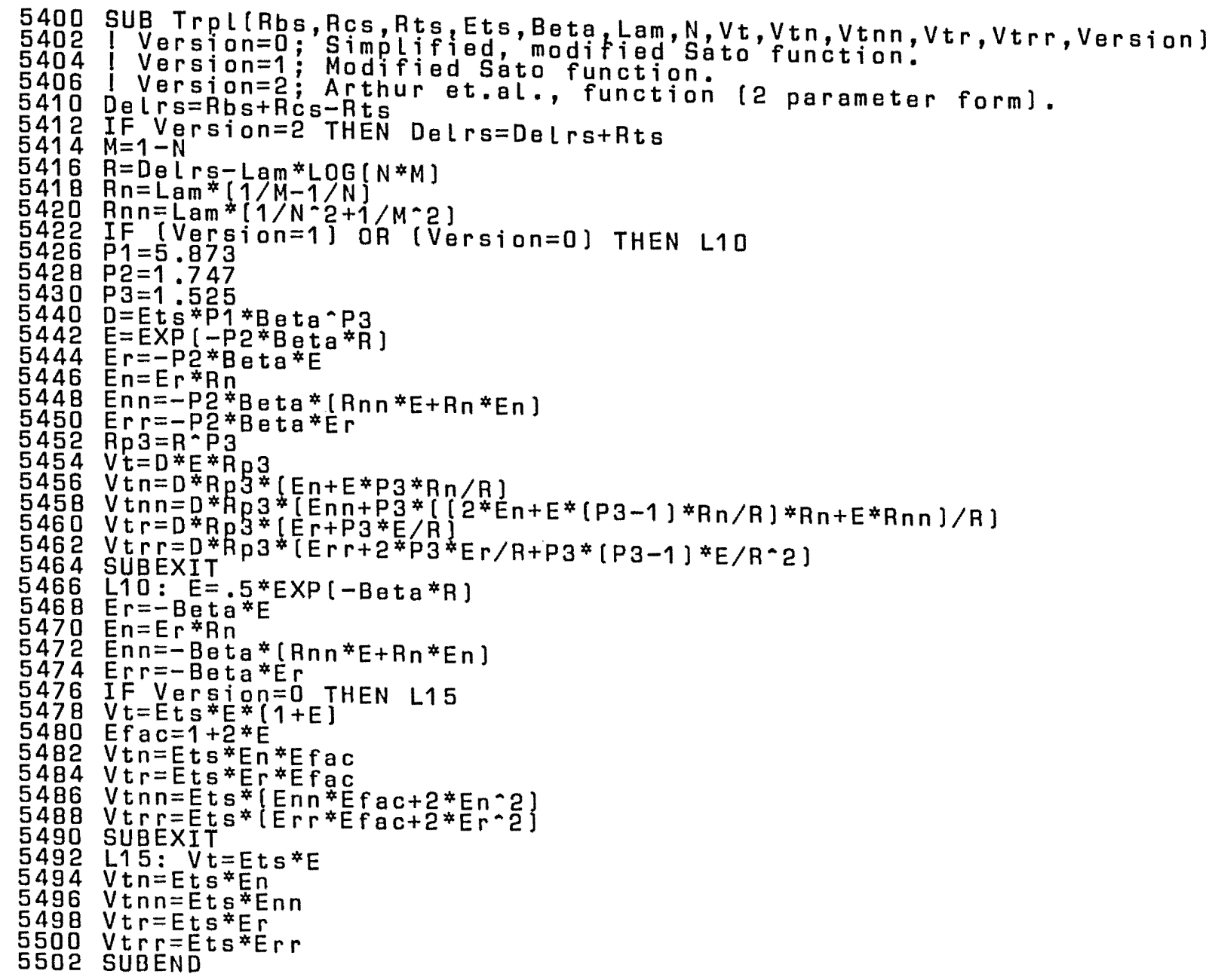




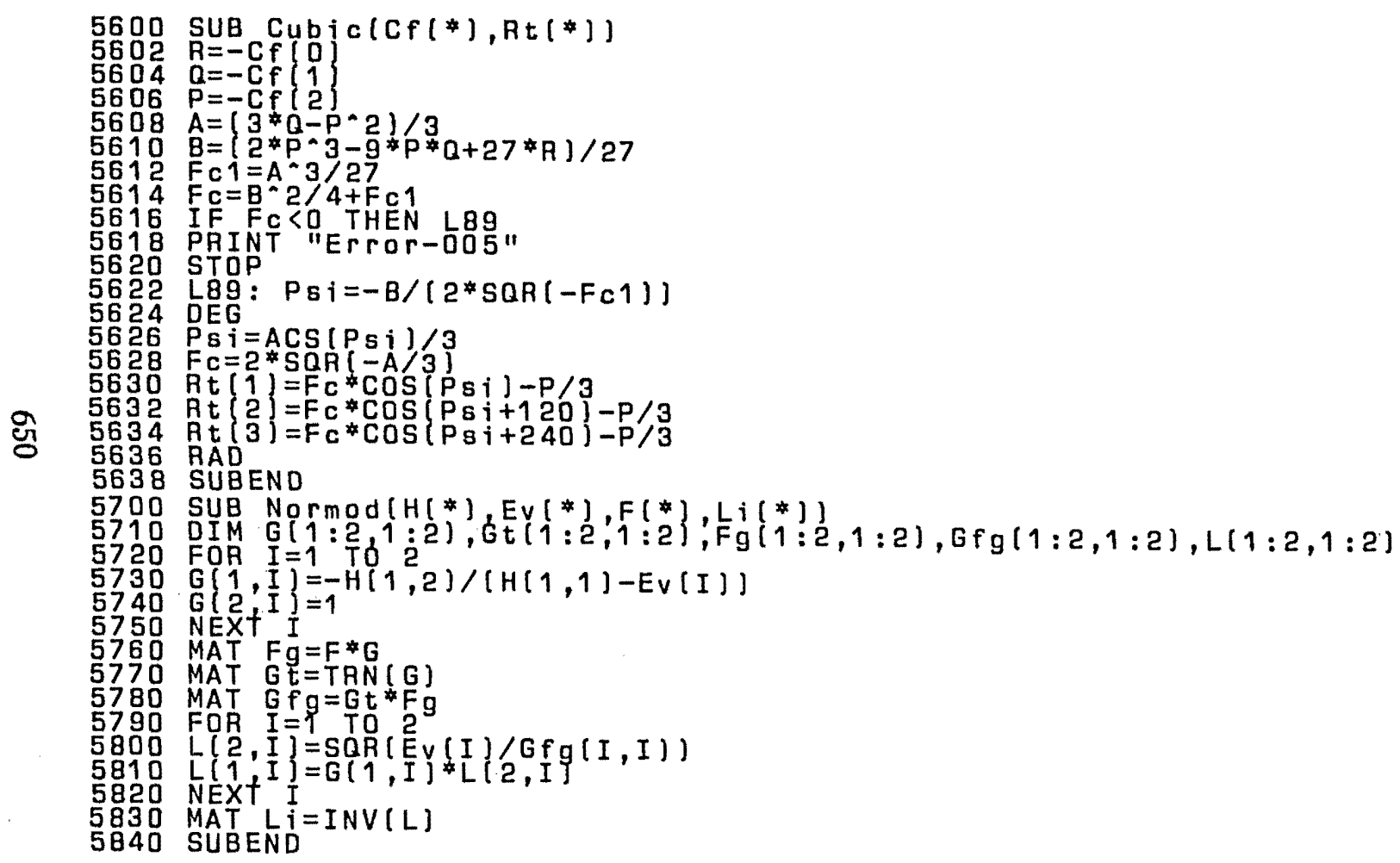




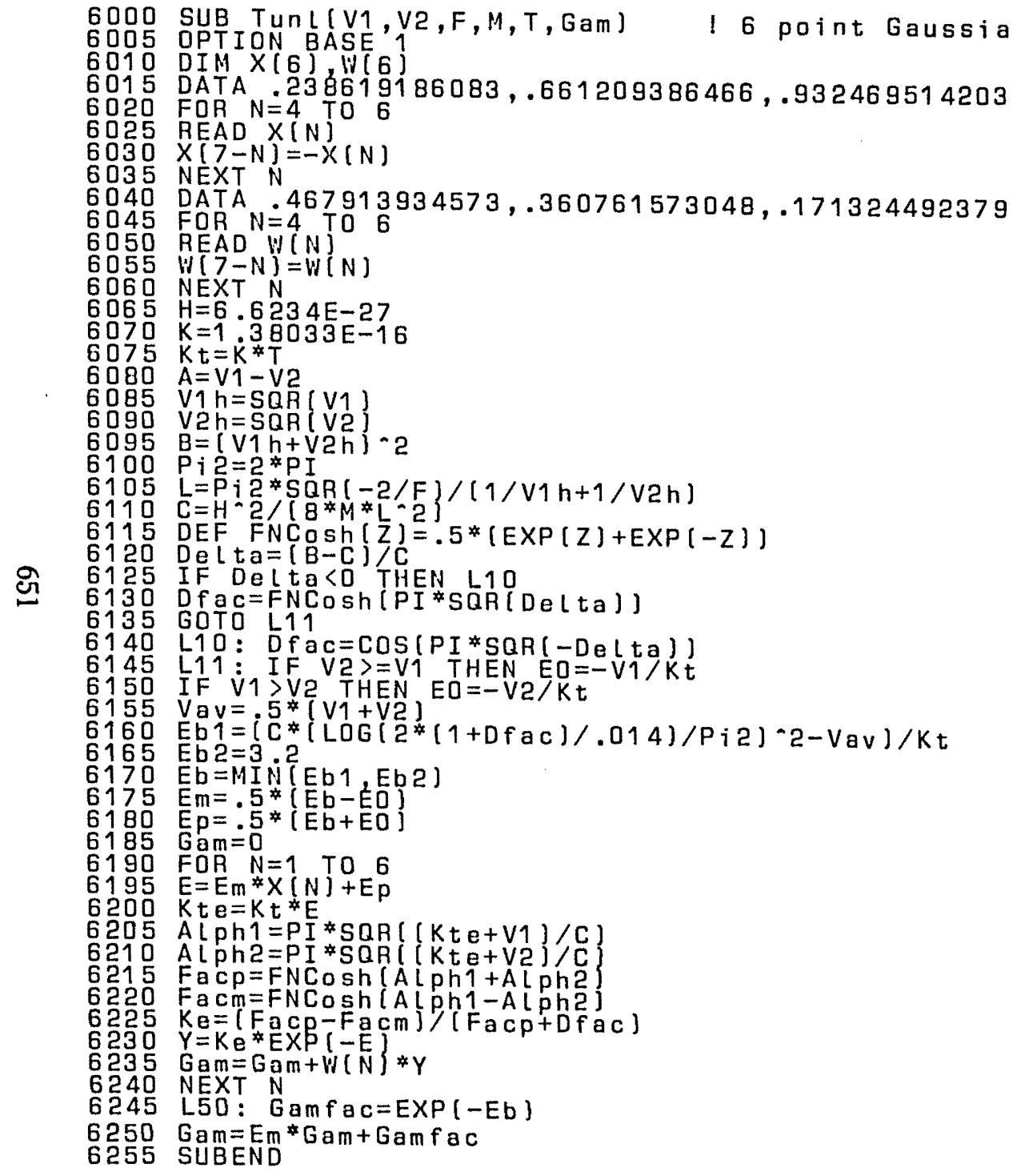




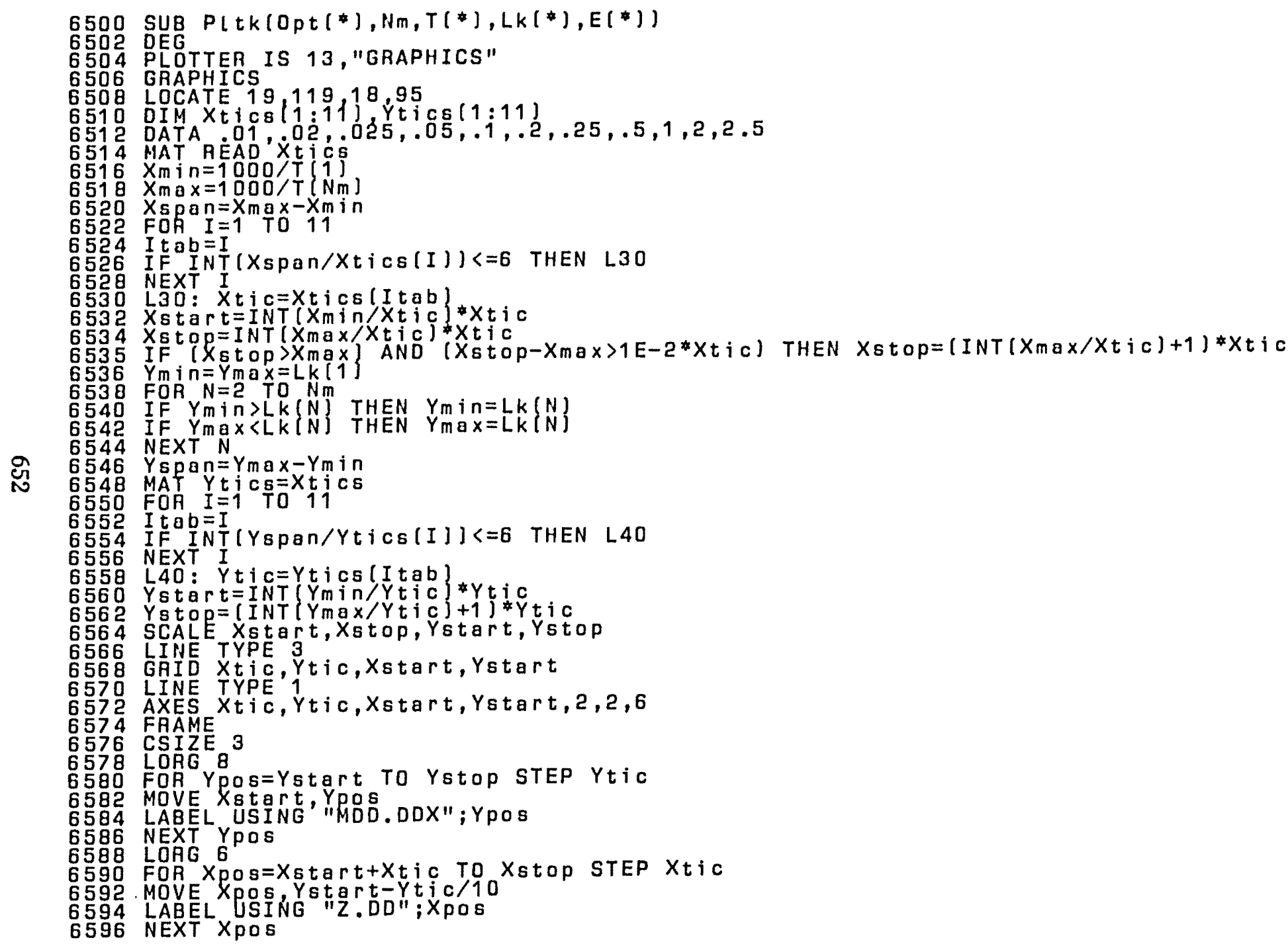




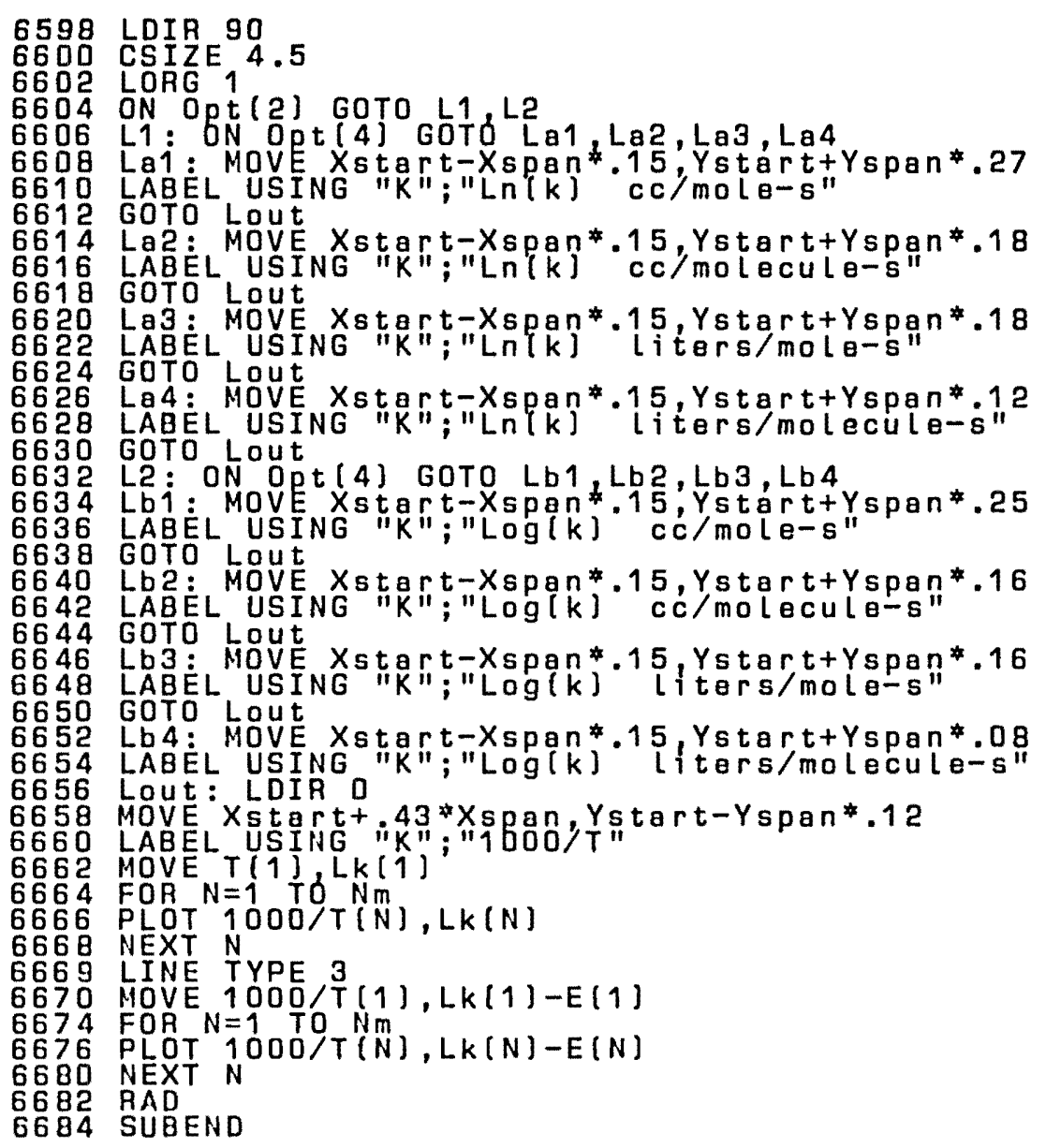




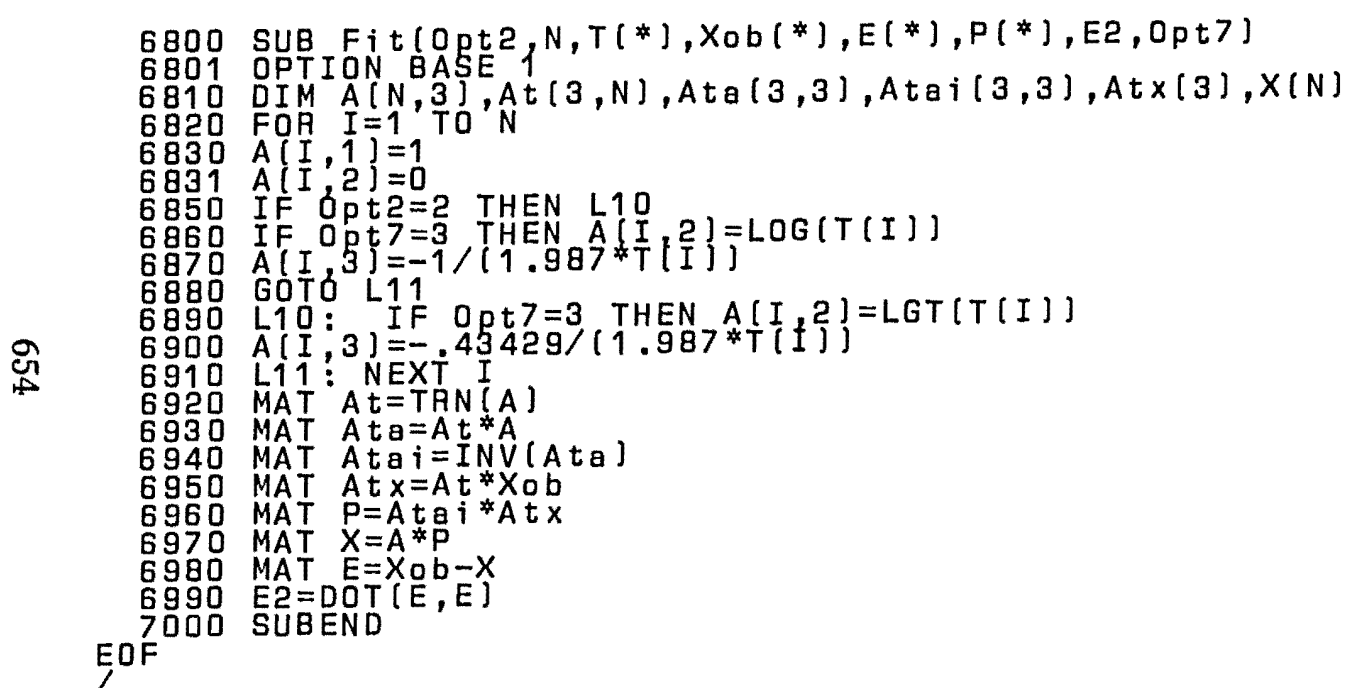

